\title{
Human latent-state generalization through prototype learning with discriminative attention
}

\author{
Warren Woodrich Pettine ${ }^{1}$, Dhruva V. Raman ${ }^{2}$, A. David Redish ${ }^{3, a}$, and John D. Murray ${ }^{1, a}$ \\ ${ }^{1}$ Yale School of Medicine, Department of Psychiatry \\ ${ }^{2}$ University of Cambridge, Department of Engineering \\ ${ }^{2}$ University of Minnesota, Department of Neuroscience \\ aThese authors share senior authorship
}

July 30, 2022

\begin{abstract}
Latent causes that give rise to experience are encountered in complex, high-dimensional feature spaces. How then do people approximate the external world with lower-dimensional internal representations that generalize to novel examples or contexts? Theories suggest internal representations could be determined by discriminative boundaries, or based on the distance from prototypes/exemplars. We developed theoretical models that use both discriminative and prototype/exemplar components to form internal representations via action-reward feedback. We then developed three new latent-state learning tasks to test human use of discrimination attention and prototypes/exemplars. The majority of subjects attended to discriminative features, as well as the covariance of features within a prototype. A minority of subjects relied on a single discriminative feature. Behavior of all subjects was captured by a model that forms prototype representations and deploys context-specific discriminative attention. These results provide insights into the human ability to generalize across causal latent states learned in high-dimensional environments.
\end{abstract}




\section{Introduction}

The high-dimensional sensory environment we experience is structured by underlying latent states ${ }^{1,2}$. Internal representations of these latent states must generalize to new observations and contexts. For example, people must not only form internal representations of nutritious and poisonous fruits, but must also generalize to all cases of discriminating between them. Previous models of latent-state learning focused on conditions where latent states are defined by the underlying reward probability associated with stimuli whose features are all informative ${ }^{2-6}$ (Table 1). However, latent states in the world often organize the vast number of feature dimensions we experience into lower-dimensional causal states (e.g., defining a poisonous fruit by its color and shape rather than by the position of the sun). How do people use experiences caused by latent states to learn generalizable internal representations? Furthermore, how does top-down attention filter distracting feature dimensions when generalizing to novel latent-state examples, or when encountering known latent states in novel contexts?

The field of category learning has proposed several models of how features can be organized into internal representations of latent states ${ }^{7}$ (Table 1). With "prototype" or "exemplar" models, new observations are categorized by their distance from either an idealized internal state prototype ${ }^{8,9}$, or individual past state examples (exemplars) ${ }^{10-13}$. Both of these models assign a new observation's internal state membership to the state with the smallest distance. Though a prototype model may use the covariance structure of features, when weighing internal state membership neither "distance" model considers a feature's informativeness for the decision process (Fig. 1A, left). An alternative approach is to define a decision boundary that separates each internal state in feature space ${ }^{14-16}$. Instead of matching a new example to the internal state it most resembles, a discriminative model defines state membership by the new example's location on a decision boundary map (Fig. 1A, right). The experimental literature finds support, depending on task demands, for prototype, exemplar, and discriminative strategies in category learning ${ }^{7}$. While there has been success in combining discriminative and exemplar components in an algorithm that learns arbitrary categories through experience ${ }^{17}$, it remains an open question how this process occurs in environments with stochastic feedback, or when categories guide action selection.

To investigate the learning of latent states in high-dimensional environments, we developed an instrumental reinforcement learning model, then designed and deployed with human subjects a series of generalization tasks. In our model, an agent forms either prototype or exemplar states. When faced with a decision, it identifies the context then allocates top-down attention to feature dimensions informative for discriminating between states in that context. In the first experiment, we found the majority of human subjects use discriminative attention when generalizing to novel latent-state examples. Individual differences in novel example generalization was accounted for in the model through reducing the use of top-down discriminative attention. This experiment establishes that humans do not exclusively rely on prototype or exemplar states. In the second experiment, we found the majority of subjects generalize by attending to features based on their consistency within a state. Those subjects were divisible into a subpopulation with random errors and a subpopulation whose errors emerged during state estimation. In the third experiment we found the majority of subjects formed states composed of multiple examples and attended to state prototype covariance. The second and third experiments also revealed a subpopulation that attends to a single discriminative feature. Results were replicated with a second online cohort after preregistration. The behavior of all subjects could be accounted for through manipulations of the model's mechanisms for discriminative attention, context recognition, and the degree to which errors arise from state estimation. These cognitive strategies may underlie individual differences in the human ability to learn internal states that flexibly generalize to novel observations in high-dimensional environments.

\section{Results}

\section{Models of prototype or exemplar state learning with flexible discriminative at- tention}

Both the prototype/exemplar (distance) models and discriminative models predict specific failure modes when generalizing action selection to novel observations or contexts. Prototype/exemplar model generalization failure is akin to distractor sensitivity. Features that have been regular (i.e. consistent) across past 
examples are considered by these models as essential for state definition - even if their regularity is incidental and not a true defining property of the latent state. When a novel example of a known latent state differs from past examples in an incidental feature, this can lead to the inappropriate creation of a new internal state (i.e., a failure to generalize an existing internal state; Fig. 1C).

Generalization failure in the discriminative model can result from overly-parsimonious inclusion of which features predict the active latent state. A discriminative model's internal representation includes only features useful for discriminating between alternatives. An agent might learn boundaries that discriminate between latent states active in separate contexts, and then encounter a novel context where all latent states are active. If the essential discriminative feature in that novel context was non-discriminative during initial learning, the agent can fail to distinguish between active latent states, thereby failing to generalize appropriately (Fig. 1D). This is related to the "continual learning" problem where artificial systems struggle to maintain representations of previous tasks as they learn new tasks ${ }^{18-21}$. Importantly, the distance model and discriminative model failure modes are complementary and dissociable: a discriminative model will successfully generalize where a distance model fails, and vice versa.

These generalization failures provide insight for our development of a "prototype states with discriminative attention" (ProDAtt) model and an "exemplar states with discriminative attention" (ExDAtt) model that learn latent states through instrumental reinforcement learning. When faced with a decision, ProDAtt or ExDAtt model agents use a discriminative component to allocate top-down attention ${ }^{22-24}$ according to which features maximally differ between states within a context. Attention-filtered features are then used to estimate the current state, which is used to select an action. Outcome feedback drives the creation of new states, the refinement of existing state representation and the learning of actions within each state (Fig. 2A). ProDAtt model agents form prototype states defined by the attention-scaled mean and covariance of the state's recent examples, while ExDAtt model agents compare a new observation to every past recent example (Fig. 2B). Other than the nature of state representation, the model algorithms are identical. (For additional details see Methods, the Supplemental interactive tutorial, or algorithmic notation in Appendix B.)

We used basic instrumental learning tasks involving latent rules and deterministic or stochastic rewards to compare the ProDAtt and ExDAtt models with six other models able to learn internal representations $2,3,5,6,17$ (Fig. S1). Of those prior models, only the actor-critic RL ANN ${ }^{25}$, the ProDAtt model and the ExDAtt model performed perfectly on all tasks.

The ProDAtt and ExDAtt models balance learning of new latent states with generalization of known states to novel examples or contexts. First, the models' states are represented and used in a way that exploits advantages of both distance and discriminative learning: state prototypes or exemplars maintain a memory of salient features available for generalization to novel contexts, while discriminative top-down attention filters salient features that could distract from generalization. Next, the models' surprise metric interacts with these mechanisms to provide a versatile threshold for estimating likely states and creating new states. The initial surprise metric used to recognize context is calculated using distance alone. This allows the model to consider all known states that could potentially generalize to a novel context. The final surprise metric is calculated after the context-specific non-discriminative dimensions have been removed. Therefore, depending on the context, the same example could either generalize to an existing state or prompt creation of a new state. Finally, reinforcement learning feedback allows the models to infer latent states in the environment by grouping examples with similar causal statistics (Fig. 2C and D).

\section{Experiment 1: Measuring discriminative attention during novel example gener- alization}

We hypothesized that the ProDAtt and ExDAtt models capture key aspects of human behavior during instrumental learning of latent states. To test this hypothesis, we used the models to design a series of tasks with specific predictions for choice behavior during learning and generalization. We implemented them in online behavioral experiments for human subjects, with initial samples recruited through Amazon Mechanical Turk, and preregistered replication samples through Prolific - online platforms extensively used for behavioral research ${ }^{26-29}$.

Given that previous models of latent-state learning generally lacked a discriminative component, we designed Experiment 1 to determine the necessity of a discriminative mechanism, and the degree to which 
it is used by human subjects. A pure distance model that defines states based on the regularity of feature dimensions will fail to generalize if a new example varies in a highly regular but non-informative dimension (Fig. 3A, left). In the same scenario, a discriminative boundary should facilitate easy generalization (Fig. $3 \mathrm{~A}$, right).

To investigate this prediction, we designed a novel example generalization reinforcement learning task (Fig. 3B). During a tutorial, subjects are instructed to expect stochastic rewards, that the same action might be rewarded for several states, and that one state might be rewarded for multiple actions. In the first block, subjects learn internal representations of latent states through taking actions and experiencing rewards. Subsequently, uncued, subjects enter a generalization block with novel examples that differ in a non-discriminative dimension. All initially learned examples have the same texture (Fig. 3C, top; Table 2). Each of these three latent states causally govern rewards for distinct actions $(A, B$ or $C)$. A fourth action $(D)$ is left unrewarded to measure exploratory behavior. The novel examples introduced in the generalization block are identical to the previous examples, except for their texture. These novel examples are associated with same latent states as their pairs in the initially learned examples (Fig. 3C, bottom; Table 2).

A pure distance model would value features by regularity across past observations. Thus, a model of pure prototype state formation predicts that the non-discriminative dimension (texture) separates initially learned examples from novel generalization examples (Fig. 3D, top). In contrast, a model with a discriminative component ignores the non-discriminative dimension when determining feature distances between examples. Thus in the decision process, initially learned examples and their associated novel generalization examples overlap in feature space after attention is applied (Fig. 3D, bottom).

To characterize generalization from choice behavior, we defined three generalization metrics: first generalization appearance, paired generalization difference, and exploration errors (Fig. 3E, see Methods).

\section{Experiment 1: Human subjects generalize to novel examples using discriminative attention}

Having designed a task to assess novel example generalization, along with metrics to assess generalization capacity, we collected two samples of subjects (initial $\mathrm{N}=109$, replication $\mathrm{N}=156$, demographics in Fig. S2). In our replication sample, we found the majority of subjects $(83 / 156)$ were within the measurement limit $(0.125)$ of perfect generalization. For the other subjects, the distribution fell off in the direction of generalization difficulties (Fig. 3F, left). The increased sample size of our replication revealed that the two groups we hypothesized from our initial sample (Fig. S5A left) instead exist as a gradient. We found a similar pattern with the paired generalization difference metric (Fig. S5A, middle and Fig. 3F, middle). These results indicate that subjects generalize to novel examples, and show inter-individual differences in that capacity.

Exploration errors assess subjects' priors over the response to novel examples. Based on the initial sample of subjects, we hypothesized that subjects would not explore action $D$ more than chance (Fig. Fig. S5A right, Fig. 3F, right and Table 3). Confirming that hypothesis, one-sided frequentist and Bayesian T-tests found all populations explored action $D$ significantly less than chance (Fig. 3F right, Table 4). This indicates subjects did not approach the novel examples with a uniform prior over possible actions.

To better understand the difference between subjects along the gradient of the first generalization appearance metric distribution, we examined learning curves within three groups. The first group were those subjects whose first generalization appearance metric was greater than the measurement limit of -0.125 , which included the majority of subjects ( $\mathrm{N}=82$, Fig. 3G, top). The second group had a metric value less than the measurement limit, but greater than the value of -0.3 that we used to distinguish groups in the initial sample of subjects ( $N=59$, Fig. 3G, middle). The third group had a metric value less than $-0.3(\mathrm{~N}=15$, Fig. 3G, bottom). We found that during the initial learning block, the learning curves of the three groups were highly similar (Fig. 3G, left column). However, during the generalization block, the learning curves for initially learned and novel examples increasingly separated as the first generalization appearance value become more negative (Fig. 3G, right column). These results show scores on the generalization metrics reflect inter-individual differences in the learning versus generalization of novel examples. 


\section{Experiment 1: Manipulation of top-down attention feedback reproduces human variation}

We used the ProDAtt and ExDAtt models to gain insight into potential sources of inter-individual differences. To do so, we introduced a parameter that linearly reduced the sensitivity of attention feedback to feature mutual information (Fig. 4A, top). The allowed us to parametrically vary the degree to which features are scaled by their discriminative informativeness (Fig. 4A, bottom). We found that ProDAtt and ExDAtt models with intact discriminative attention learned the initial states and generalized to novel examples (Fig. 4B, single agents shown in Fig. S6). Distortion of attention feedback had no impact on learning curve trajectories during the initial block. However, as the distortion of attention feedback increased, generalization block learning curves of initially learned and novel examples increasingly separated (Fig. 4B). For both ProDAtt and ExDAtt, increasing distortion led to an increase in the magnitude of the first generalization appearance difference, an increase in the magnitude of the paired generalization difference, and slight increases in exploration error - though it remained below chance (Fig. 4C and D). Thus, deficits in the use of top-down attention in the models recapitulate human inter-individual differences in novel example generalization.

\section{Experiment 2: Dissociating attention models through novel context generaliza- tion}

Having established human subjects' use of discriminative attention when it is advantageous, a natural next question is whether humans continue to use discriminative attention during learning when it is disadvantageous. Furthermore, in subjects who predominantly learn discriminative boundaries, and two states only partially overlap in defined boundaries, which set of boundaries are used? To investigate these questions, we designed a context generalization task where latent state representations must be learned separately (different learning contexts), and then are all active in a novel context. To successfully generalize when the latent states are all active, subjects must attend to a feature that was non-discriminative when the latent states representations were initially learned (Fig. 5A).

Experiment 2's novel context generalization task version $1\left(\mathrm{CG}_{1}\right)$ is structured in three blocks (Fig. 5B). During the first block (learning context 1), subjects learn to represent latent states separable by two dimensions (color and shape), each rewarded for a distinct action (action $A$ for magenta circles and action $B$ for orange squares). After block 1, subjects are instructed to put aside what they had learned and to learn entirely new states along with entirely new actions (action $C$ and action $D$ ). In block 2 (learning context 2) latent states are distinguished only by single dimension (shape: circle with action $C$, and square with action $D$ ). Finally, during block 3 (novel generalization context), subjects are instructed that all actions are available, and they will encounter all previously seen stimuli (exemplars described in Table 5).

If subjects over-rely on discriminative boundaries during learning, they will exhibit a specific error type which we call a "discriminative error." This error occurs when, due to exclusion of a previously nondiscriminative feature dimensions, the model feature distance between two perceptually distinct examples is zero (two circled states in Fig. 5A, overlap of examples in Fig. 5C, top row). If they are primarily attending to discriminative features, they could either attend to the feature discriminative across both contexts D1M, or include the two features from context 1 when considering those latent-state representations D2M.

In contrast, a model attending to prototype covariance will notice the features common in each context, even if the features are non-discriminative. This results in clear separation between internal states (Fig. $5 \mathrm{C}$, bottom left), which in turn facilitates block 3 novel context generalization. A model that attends to all features will similarly distinguish between states learned in different contexts (Fig. 5C, bottom right).

\section{Experiment 2: Majority of subjects generalize in novel context and attend to initially non-discriminative features}

Analyzing human subject behavior ( $\mathrm{N}=53$, demographics in Fig. S7, top), we looked at the proportion of examples for which there was an error during their initial appearance during the generalization context (block 3). This "initial generalization error" provides an indication of subjects' ability to generalize when all latent states are active. The majority of subjects (39/53) generalized better than chance (full statistics in Table 6). Nonetheless, there was a large degree of individual variability. We therefore visually identified 
a threshold for subjects at the initial generalization error value of 0.4. (Fig. 5D, top). Examining the proportion of discriminative errors, we found similar variation and visually identified a discriminative error threshold of 0.6 (Fig. 5D, bottom).

To characterize subjects' use of attention during generalization, we used idealized versions of the attention models (D1M, D2M, prototype covariance, and attention to all features). Each of these models produce attention weights that can be used to quantify a distance between a presented example and the internal representations of each example (Fig. S9A and B, Fig. 5E).

We computed the confusion matrix for all subjects pooled together, as well as for three subpopulations derived from the thresholds on initial generalization error and discriminative error (Fig. S9C). Confusion matrices of the subpopulations show visual similarity to distinct predictions of different idealized attention models (Fig. 5F). We quantified the correspondence using Bayesian regression to fit the idealized confusion matrices to the observed confusion matrices (Bayesian regression model structure shown in Fig. S10A, model recovery analysis in Fig. S12, posterior predictive distributions in Fig. S11). We also used Pareto smoothed importance sampling leave-one-out cross-validation (LOO) ${ }^{30,31}$ to compare the models with all attention predictors to models where a single predictor (e.g. prototype covariance) is removed.

We found that subjects above the discriminative error threshold had the highest coefficient values for the discriminative attention models (Fig. 5G, middle/left, Table 7). Subjects below the discriminative error threshold and above initial generalization error threshold had the highest coefficient value for random errors, with significant coefficients for the distance models (prototype covariance attention and all-feature attention (Fig. 5G, middle/right). Subjects below both the discriminative error and initial generalization error thresholds had the highest coefficient value for the all-feature attention model (Fig. 5G, right). Model comparison revealed similar trends (Fig. S13, Table 8).

This analysis revealed subpopulations with distinct attention to features during novel context generalization. Furthermore, most subjects $(45 / 53)$ composed a subpopulation that attended to the initially non-discriminative features.

\section{Experiment 2: Subpopulation attending to discriminative features learns single boundary for all states}

For the subpopulation with high discriminative errors, we then examined whether subjects used a D1M or D2M model of attention. We found no overlap in the coefficients' 97\% CI (D1M: 0.406, 0.721; D2M: $0.000,0.367$ ). Similarly, removing the D1M predictor resulted in much worse cross validated performance (LOO mean $=4.41, \mathrm{SE}=4.88)$ than did removing the D2M (LOO mean=15.39, $\mathrm{SE}=4.49$, Fig. $5 \mathrm{H}$, left, Table 8). To disentangle the effect of each predictor, we then calculated the partial correlation coefficients for the D1M and D2M. We found the partial correlation distribution of the D1M to be higher than the D2M, and with only $3 \%$ overlap (Fig. $5 \mathrm{H}$, right). Taken together, these results indicate that subjects with high discriminative errors converged on a single discriminative feature boundary for all states, rather than learning discriminative boundaries for each state.

\section{Experiment 3: Majority of subjects learn states that include multiple examples}

Experiment 1 found individual variation in subjects' use of discriminative attention when generalizing to novel examples. Experiment 2 found that during generalization to novel contexts, the majority of subjects attended to features regardless the feature's initial discriminative informativeness, while a minority composed a distinct subpopulation that attended to a single discriminative feature. However, Experiment 2's $\mathrm{CG}_{1}$ was not designed to differentiate between alternatives to the discriminative attention model (note similarity of prototype covariance and all-feature attention confusion matrices in Fig. 5E).

For the novel context generalization task version $2\left(\mathrm{CG}_{2}\right)$ of Experiment 3, we modified the configuration of stimulus features to better differentiate types of state formation, while still enabling detection of a discriminative model of attention (Table 9, Fig. 6A, Fig. S16A and B). In this task, attention to prototype covariance indicates learning states that include multiple examples, whereas exclusively attending to all features indicates learning separate states for each individual example. After collecting an initial sample ( $=49$, mTurk), we preregistered our results and collected a replication sample ( $N=108$, Prolific) (demographics in Fig. S7 middle and bottom). 
Similar to Experiment 2, we found the majority of subjects generalized better than chance $(81 / 106)$. We also found individual variation in the proportions of initial generalization and discriminative errors (initial sample in Fig. S18A and Table 10, preregistered replication in Fig. 6B and Table 11). Using the initial sample of subjects, we identified and then preregistered thresholds on both metrics (Fig. S18A, Table 10). We used the idealized attention model feature distances (Fig. S16A) to predict confusion matrices (Fig. S16B and Fig. 6C). For initial and replication samples, we computed the confusion matrix for all subjects pooled, as well as each subpopulation (Fig. S16C and D, Fig. 6D). We then performed a Bayesian regression (Bayesian regression model in Fig. S10B, model recovery in Fig. S17, posterior predictive fits in Fig. S19 and Fig. S20, initial sample fit results in Table 12).

We tested our preregistered hypotheses regarding the existence of subpopulations defined by the types of errors made during generalization. To interpret findings, we used Bayesian regression coefficients and LOO model comparison. Behavior of the subpopulation above the discriminative error threshold was best described by the discriminative attention model (Fig. 6E middle/upper, Table 14). Within that subpopulation, subjects above and below the initial generalization error threshold showed the same pattern for discriminative attention in both fit coefficients and model comparison (Fig. S21A and B). While subjects above and below the initial generalization error threshold had distinct learning curves (Fig. S22C), our results indicate they attended to the same feature during generalization.

Next, we hypothesized that subjects below the discriminative error threshold would attend to a combination of prototype covariance and all-features. Additionally, for those below the discriminative error threshold but above the initial generalization error threshold, we hypothesized that their errors would primarily be random (i.e., not correspond to state estimation). In this subpopulation, the coefficient for the discriminative model contained zero in its interval, while those for prototype covariance and attention to all features were above zero, confirming the hypothesized pattern. Furthermore, we found this subpopulation had the highest coefficient value for the intercept term (Fig. 6E middle/lower), and that the "Null" model, with only the intercept, overlapped in its distribution with models containing other predictors (Fig. S21B, Table 15). Therefore, as hypothesized, errors committed by subjects below the discriminative error threshold but above the initial generalization error threshold, were largely random.

For the subpopulation below both thresholds, the prototype covariance predictor and the all-features predictor were the only coefficients significantly above zero (Fig. 6E bottom, Table 14), and removing either predictor had a large effect on cross-validated model comparison (Fig. S21B, Table 15). This further indicates that subjects below the discriminative error threshold are differentiated by the initial generalization error threshold. It also supports our preregistered hypothesis that subjects below the discriminative error threshold produce a pattern of novel context errors well described by a combination of attending to all available features and features with strong prototype covariance.

In Experiment 3, we found the majority of subjects attended to features based on their role in defining a state composed of multiple examples (70/106), while a minority attend to a single discriminative feature $(36 / 106)$.

\section{Manipulating algorithmic model context recognition recapitulates discriminative attention subpopulation behavior}

Across Experiments 2 and 3, we consistently found a subpopulation of subjects whose errors indicate they primarily attend to the discriminative feature. To gain insight into potential reasons for their behavior, we manipulated the ProDAtt and ExDAtt models' context recognition mechanism (single agents performing the $\mathrm{CG}_{2}$ in Fig. S23).

First, we considered the hypothesis that subjects share an underlying state representation system, but differ in the use of context information during learning. Both the ProDAtt and ExDAtt models use a threshold on surprise to determine the states active in a context. As that threshold increases, the number of potential states to consider increases (Fig. 7A). In both the ProDAtt and ExDAtt models, we found that increasing the context surprise threshold resulted in more initial generalization errors, more discriminative errors, and a shift in the coefficient values to favor the discriminative attention predictor (Fig. 7B and C, learning curves, confusion matrix in Fig. S24). This is because when there is an appropriate context surprise threshold, the ProDAtt and ExDAtt models recognize that the latent states governing rewards in learning context 1 are different from those governing rewards in learning context 2. Thus, the state creation mechanism 
causes ProDAtt and ExDAtt models to create new internal state representations associated with the learning context 2's latent states. However, as the context surprise threshold increases, agents become more likely to believe that the latent states from learning context 1 continue to govern causal rewards in learning context 2. Instead of creating new states in the second context, they simply update the values for actions $C$ and $D$. When these agents then reach the novel generalization context (block 3) they fail to distinguish between context 1 and context 2 latent states. Interestingly, this results in a distinct transition point separating agents who create distinct state representations (appropriate context recognition, low discriminative errors), from those who do not (inappropriate context recognition, high discriminative errors). This is similar to the stark difference in confusion matrices between human subpopulations with low versus high discriminative errors.

We also trained an ANN implementation of actor-critic reinforcement learning on the $\mathrm{CG}_{1}$ and $\mathrm{CG}_{2}$ while manipulating the hidden-layer weight initialization (Fig. S25, Fig. S26, Fig. S27). The ANN produced discriminative errors and attended to a single discriminative feature regardless of initialization. At high initialization variance, its hidden-layer representation and behavior made it qualitatively equivalent to an ExDAtt model lacking context recognition (see Appendix C).

\section{Exemplar and prototype latent-state learning models dissociable in their atten- tion to prototype covariance}

Given that the two subpopulations of subjects below the discriminative error threshold primarily differed in the significance of the intercept term (i.e. random behavior), we hypothesized that the state estimation process would provide mechanistic insight. To investigate this, we introduced to the ProDAtt and ExDAtt models a probability of error on every trial of the generalization context. We then manipulated the degree to which that error was random versus influenced by state distance. ProDAtt and ExDAtt agents' internal state representations exist at locations in feature space such that some states are closer (i.e. more similar) than others (Fig. 8A, top). On the error trials, we used a softmax $\beta_{\text {Confusion }}$ parameter to vary the degree to which errors are random versus dictated by proximity in feature space (Fig. 8A, bottom). Random errors (low $\beta_{\text {Confusion }}$ ) could be due to a variety of causes (e.g., issues in use of action values, forgetting of task structure, task disengagement). However, errors due to the distance between states in feature space (high $\left.\beta_{\text {Confusion }}\right)$ can only arise from errors in state estimation.

We trained ProDAtt and ExDAtt agents on the $\mathrm{CG}_{2}$ while increasing the value of softmax $\beta_{\text {Confusion }}$. In the ProDAtt model, we found that increasing $\beta_{\text {Confusion (less random) had no effect on initial generalization }}$ errors (as expected by implementation) or discriminative errors (Fig. 8B, left and middle). However, increasing $\beta_{\text {Confusion }}$ reduced the coefficient value for the intercept (indicating random errors). The coefficient values increased for both the prototype covariance attention and attention to all features, with the prototype covariance coming to dominate (Fig. 8B, right). The coefficient for the all-feature attention predictor is high because ProDAtt model attends to the values of features when comparing them to the prototype mean. Whereas prototype covariance can limit the field of attention, the prototype mean causes state exemplars to produce similar feature-driven responses (see ProDAtt confusion matrices in Fig. S28D).

In the ExDAtt model, increases in the softmax $\beta_{\text {Confusion }}$ also had no impact on initial generalization error (again, as expected, Fig. 8C, left). However, increasing $\beta_{\text {Confusion }}$ reduced ExDAtt discriminative errors (Fig. $8 \mathrm{C}$, middle). When fitting the ExDAtt confusion matrix, increasing $\beta_{\text {Confusion }}$ reduced the random intercept, increased the coefficient for prototype covariance, and caused the coefficient value for the all-feature model to dominate (Fig. 8C, right). Given that the ExDAtt model does not compute the prototype covariance, why is there a significant value for this predictor? When an exemplar model considers an examples' state membership it computes the distance for each state exemplar. These individual distances are combined to arrive at the distance used in state estimation. While one might expect an exemplar model to behave as if it memorized each example, instead the pooling of distances among state exemplars causes a similar response to all state exemplars (Fig. S28H). Indirectly, this allows the ExDAtt to achieve something similar to the prototype mean. While the ExDAtt model does not use prototype covariance, including the idealized prototype covariance attention model with the all-feature attention model allows it to partially capture this effect.

Taking inspiration from ProDAtt and ExDAtt model behavior, we hypothesized the use of a "state-bias" idealized attention model that assumes all state exemplars are biased by the feature distance other state 
exemplars (Fig. 8D, Bayesian regression model in Fig. S10C). We fit a regression model with predictors for prototype covariance and state bias to ProDAtt and ExDAtt model errors at each value of softmax $\beta_{\text {Confusion }}$, and performed LOO model comparison with the prior model containing terms for discriminative attention and attention to individual features. Both ProDAtt and ExDAtt model saw significant improvements in LOO for the regression including state bias (Fig. 8E, left and Fig. 8F, left). As $\beta_{\text {Confusion increased, ProDAtt }}$ and ExDAtt models showed distinct patterns: whereas ProDAtt has increased coefficients for prototypecovariance and state-bias predictors (Fig. 8E, right), ExDAtt only had increase for the state-bias predictor (Fig. 8F, right).

Using the prior regression model (prototype-covariance + discriminative + all features), both ProDAtt and ExDAtt models qualitatively reproduced the error patterns of all subjects below the discriminative error threshold. However, by considering the mechanisms giving rise to the pattern of behavior, we formulated an alternative model (prototype covariance + state bias) that both better captured ProDAtt and ExDAtt model behavior, and cleanly distinguished between the use of prototype versus exemplar states.

\section{Experiment 3: Majority of human subjects attend to common state features and prototype covariance}

Having found a combination of idealized attention models (prototype covariance with a bias towards state features) that both cleanly distinguished between the ProDAtt and ExDAtt model hypotheses, and better fit their behavior, we applied that regression as an exploratory (i.e., not preregistered) analysis of a preregistered hypothesis (use of prototype covariance) using the Experiment 3 initial and replication sample subjects (posterior predictive fits in Fig. S29).

We found that the behavior of subjects above the discriminative error threshold was fit significantly worse by the new regression model (Fig. 8G for initial sample and Fig. 8J for replication sample). This is expected, as the new regression model did not contain a predictor for discriminative attention. The coefficients for subjects below the discriminative error threshold and above the initial error threshold were significant for both prototype covariance and state bias. However, model comparison revealed the fit was equivocal between the prior regression model and the new regression model (that included state bias) (Fig. $8 \mathrm{H} / \mathrm{K})$. This provides further indication these subjects' errors arose both from both state estimation and other sources. The behavior of subjects below the discriminative error threshold and below the initial error threshold was significantly better fit by the new regression model. Importantly, in both the initial and replication samples, this subpopulation had significant coefficient values for the prototype covariance predictor (Fig. 8I/L, all fit results in Table 16 and model comparison results in Table 17).

These results support that the majority of subjects attend to prototype covariance (70/106). Notably, only the ProDAtt model recapitulates the pattern of behavior by subjects below the discriminative error threshold.

\section{Discussion}

Our results indicate that the majority of human subjects use both discriminative attention and prototype covariance when generalizing latent states learned through taking actions and observing outcomes. Moreover, individual deviation from that behavior could be accounted for through manipulations of a model that learns prototype states and deploys context-specific discriminative attention.

The theory underlying our findings draws heavily from previous work in latent-state reinforcement learning and category learning. Both fields have developed models that learn representations of the environment through experience. Why did these models fail at the basic instrumental latent-state learning tasks? Previous latent-state learning models focused on detecting shifts in the latent causes of Pavlovian conditioning or rewards from a bandit task, not the learning of latent rules. Thus, the Pavlovian models ${ }^{5,6}$ struggle with credit assignment when multiple latent-states are potentially active on any given trial, and the bandit models ${ }^{2,3}$ struggle to detect the features separating high-dimensional states. As a result, both the Pavlovian and bandit models settled on state representations with incorrectly low estimates of action values. The SUSTAIN algorithm for category learning ${ }^{17}$, on the other hand, excelled at learning latent rules through deterministic feedback (and provides a compelling account for neural activity during deterministic latent- 
state learning $\left.{ }^{32,33}\right)$. However, the SUSTAIN model's task performance deteriorated when feedback became stochastic. The reason has to do with the difference between "categories" and "states." A category is the outcome of a classification process (i.e. state estimation alone). A state, however, defines a setting for experiences and actions. Thus, the SUSTAIN model excelled with deterministic rewards when action selection could be reduced to state estimation. However, it has no mechanism for learning action values or how to handle stochastic feedback. Our ProDAtt and ExDAtt models combine advantages from category learning for state estimation with advantages from latent-state learning for action selection and stochastic outcomes.

Though the ProDAtt model better captured the full range of subject behavior, that does not necessitate that humans learn prototype states rather than exemplar states or decision boundaries. Indeed, a version of the ExDAtt model that includes prototype covariance (a mixed prototype-exemplar model) could produce errors similar to the ProDAtt model, while maintaining the exemplar model benefit of nonlinear category membership. A neural network with the ability to modify decision boundaries based on context would generalize to novel settings ${ }^{20,34}$. Furthermore, extensive work in the field of category learning has provided rigorous analytical tools for dissociating between prototype, exemplar and decision boundary models, demonstrated variations of the models that mix different components, and shown that humans use different models depending on task structure (see summary of citations in Table 1). The ProDAtt model is useful in that it allows us to investigate a system that behaves similarly to human subjects on the tasks, and for which we have full access to both study and manipulate the core mechanisms.

While both ProDAtt and ExDAtt models create a context by assembling a set of the most likely states, humans likely use a variety of context recognition strategies. Substantial research has been done on using reward statistics to infer context ${ }^{3,35}$. The results we present on how failures in context recognition impact latent-state learning and generalization should generalize across context recognition strategies.

Aside from our work's theoretical implications, the behavioral results provide a rich foundation for future studies of individual differences in attention and during generalization. While we find compelling differences between the subpopulations identified in Experiments 2 and 3, it is likely - as parametric manipulation of the ProDAtt indicates - that they exist along a continuum. Several studies have found deficits in how those with autism spectrum disorder (ASD) learn latent rules that govern the informativeness of cue-predictive features $^{36-38}$. Those with attention-deficit/hyperactivity disorder (ADHD) demonstrate particular issues with the maintenance of state information and with top-down attention ${ }^{39-41}$. Furthermore, there is high diagnostic overlap between ASD and $\mathrm{ADHD}^{42-44}$. Our novel example generalization and novel context generalization paradigms provide a powerful platform for transdiagnostic research of ASD and ADHD, as well as other conditions involving attention or generalization, such as psychosis.

Our experimental and theoretical results can be extended in multiple directions. During the initial learning contexts of Experiment 2 and 3 (when there were two actions available), subjects in all subpopulations approached perfect performance. However, when there were four actions available during the novel generalization block, or during the entirety of Experiment 1, subjects seemed to achieve a lower steady state performance level. ProDAtt and ExDAtt models can achieve perfect performance, even with four actions. Previous work in reinforcement learning has found the working memory system can sustain two actions, but four causes a shift to a high-load regime ${ }^{45-48}$. These working memory effects are likely the source of reduced subject performance we observed in similar task conditions. Given the multiplicity of possible mechanisms involved in working memory, our manipulations of context recognition and state-estimation errors in the ProDAtt and ExDAtt models are both generalizable across mechanisms and provide initial insights into where to look for the source of working memory limitations. For example, working memory limitations could arise from the maintenance of action values, or the maintenance of state representations. Our manipulation of artificially induced errors indicates that subjects above threshold on both Experiment 3 metrics generated errors primarily during the state estimation process. That points to limitations in the maintenance of state representation, rather than actions values. For the subpopulation with highly random errors, future studies could be designed that use reaction times to gain insight on whether errors arose during action selection, state estimation or both.

In this work we investigated individual differences in the use of attention during instrumental latentstate learning and generalization. Our findings suggest future theoretical and experimental investigations in reinforcement learning and category learning, as well as paradigms useful for studying individual differences observed in psychiatric conditions. 


\section{Acknowledgments}

This research was partly supported by NIH grants R01MH112746 (JDM), R01MH112688 and P50MH119569 (ADR), as well as by an appointment to the Intelligence Community Postdoctoral Research Fellowship Program at Yale University, administered by Oak Ridge Institute for Science and Education (ORISE) through an interagency agreement between the U.S. Department of Energy and the Office of the Director of National Intelligence (ODNI) (WWP). We thank Vishwa Gouder, Adriana Di Martino, Daniel Ehrlich and Matt Jones for comments and discussion. 


\section{Methods}

\section{Previous models that learn latent state, categories or policies.}

We tested several previous representation (latent-state, category or policy) learning models on their ability to learn latent rules through instrumental feedback. The prior models of latent-state learning included the Redish 2007 model $^{2}$, the Gershman and Niv 2012 model $^{3}$, the Gershman et al. 2017 model $^{5}$ and the Cochran and Cisler 2019 model $^{6}$. To implement these latent state models, we used a code package made publicly available by Cochran and Cisler $^{6}$. We also implemented the SUSTAIN model ${ }^{17}$ from category learning using code originally written by Todd Gureckis, and an artificial neural network actor-critic reinforcement learning model based on standard practices ${ }^{25}$.

\section{Models of prototype or exemplar states with discriminative attention}

Overview Our "prototype states with discriminative attention" (ProDAtt) and "exemplar states with discriminative attention" (ExDAtt) models are extensions of a previous latent-state reinforcement learning model used to study the phenomenon of extinction and renewal ${ }^{2}$. The ProDAtt and ExDAtt models balance the learning of internal agent states $(s)$ that represent latent states in the environment with the learning of actions within those states $(Q(s, a))$. To accomplish both state estimation and new-state creation, the algorithms use a clustering method where newly encountered cue examples $(c(t))$ are either classified as a member of the least-surprising state (a.k.a. cluster), or if the surprise for any state $(\mu)$ exceeds a threshold $(v)$, it forms a new state. In the ProDAtt model, surprise is calculated as the distance from a central mean, while the ExDAtt model performs a weighted average of the distance from all past examples. The clustering is a two-step process. In the first step, a context is formed composed of states $(\{i \in S\})$ whose surprise does not exceed a certain threshold $\left(v_{C}\right)$. In this stage, the ProDAtt weighs features for each state by their precision matrix $\left(I_{P}\right.$, the inverse of the prototype covariance matrix), while the ExDAtt model uses all features. The mutual information (MI) of each feature is then computed according to a feature's capacity for maximally differentiating between states in the context. This MI is used to create a set of top-down attention weights $\left(I_{M I}\right)$. The use of attention is modulated by reward history $(\bar{\delta})$ such that consistent deviation from expected reward increases the overall level of attention to features in the environment. After modulation, the attention weights are applied and surprise is recalculated. Each state then learns its own set of action-values through a Rescorla-Wagner update. Our overview is expanded in what follows. Furthermore, an interactive tutorial is available both online (https://github.com/murraylab/ProDAttExDAttModelIntuition) and in the supplementary code.

\section{Computation of surprise - ProDAtt model}

When an example cue vector is encountered, the ProDAtt model first computes the distance from the prototype $\mu$ for each state $i$ using the equation,

$$
Z_{i}((c t))=w_{A}\left(1-\xi_{\text {noise }}\right)\left(c(t)-\mu_{i}\right)
$$

where $\xi_{\text {noise }}$ (set to 0.98 ) represents any consistent factor in the system that reduces representational precision, while vector $w_{A}$ implements top-down attention. It then uses the vector $Z_{i}(c(t))$ to calculate a single radial distance from the internal agent-state prototype with the equation,

$$
D_{i}^{2}(c(t))=Z_{i}(c(t))^{\prime} I_{P, i} Z_{i}(c(t))
$$

where $D_{i}^{2}(c(t))$ is the radial distance and $I_{P, i}$ is the precision matrix (inverse of covariance $\Sigma_{i}$ ) for that state. Given there is no history dependence in the states of the task, we assumed a uniform prior over the probability of observing specific internal-states. Thus,

$$
P\left(s_{i} \mid c(t)\right) P(c(t))=\frac{1}{\sqrt{s \pi^{n_{c}}\left|\Sigma_{i}\right|}} \exp \left(-\frac{1}{2} D_{i}^{2}(c(t))\right),
$$

and 


$$
P\left(c(t) \mid s_{i}\right) \propto P\left(s_{i} \mid c(t)\right) P(c(t)) .
$$

It then calculates the surprise index by taking the negative log of each posterior ${ }^{49}$ such that,

$$
F\left(s_{i}\right)=-\ln \left(P\left(c(t) \mid s_{i}\right)\right)
$$

\section{Computation of surprise - ExDAtt model}

To create an "exemplar states with discriminative attention" (ExDAtt) model, we modified the calculation of surprise by the ProDAtt model, such that it is a weighted combination of each example's surprise. When implementing this, we looked to concepts described in the generalized context model $(\mathrm{GCM})^{10-13}$, a commonly used exemplar model of categorization. In the GCM, a distance metric is computed between a new stimulus and each example of a category. A nonlinearity is applied to amplify that distance and then category distance is determined by averaging its individual example distances.

In the ExDAtt model, surprise is computed for each example within the recent history for a state, and then an average is weighted by the frequency of each example. This is determined using the equation,

$$
F\left(s_{i}\right)=\frac{\sum_{j} n_{j} F\left(s_{i, j}\right)}{n},
$$

where $n_{j}$ indicates the number of times an example is observed, and $n$ the total number of examples.

\section{Identifying a context}

When presented with example vector $c(t)$, an agent first compares it to the bank of known states using equation 1 , with $w_{A}$ set to a vector of all ones. The agent compares the level of surprise for each state to a fixed threshold $v_{C}$. States whose surprise is below the threshold are included in the candidate set of states.

\section{Creation of new states}

If, at any stage of the process, no state's surprise is below the specified threshold, the agent creates a new state:

$$
\begin{aligned}
& N=\left|\left\{i: F\left(s_{i}\right)<v\right\}\right| \\
& \text { if } N<1, \text { create new state, }
\end{aligned}
$$

where $v$ defines the surprise threshold for new state creation. The initial $\mu_{i}$ is centered on the novel observation. New internal-states are given a "burn in," time of $n$ trials before these values are updated. When a ProDAtt model agent creates a state, the new state's precision matrix $I_{P}$ is initialized to 25 along the diagonal.

\section{Discriminative attention}

If the set of states for a context is greater than one, the agent performs a discriminative step to identify attention weights. This is done by calculating the MI of each individual cue, $k^{50}$. First the agent calculates the entropy of cue $k$ for each state $\{i \in S\}$ using the equation,

$$
H\left(C_{i, k}\right)=p\left(s_{i}\right) \sum_{t \in s_{i}} p\left(c_{k}\right) \log _{2}\left(p\left(c_{k}(t)\right)\right)
$$

where $C_{i}$ is a matrix of all past trials' $(t)$ cue vectors in the memory of state $s_{i}$, and $C_{i, k}$ is a slice of feature $k$ for each example. The agent then calculates the total entropy of each cue with the equation,

$$
H\left(C_{k}\right)=\sum_{t} p\left(c_{k}\right) \log _{2}\left(p\left(c_{k}(t)\right)\right)
$$


where $C_{k}$ is a vector containing all memories of feature $k$ across every trial $t$ for states $\{i \in S\}$. The agent then uses these values to calculate the MI of each cue with equation,

$$
I_{M I}=I\left(C_{k}, S\right)=H\left(C_{k}\right)-\sum_{i} H\left(C_{i, k}\right) .
$$

$I_{M I}$ is a vector containing the MI for each feature across states $\{i \in S\}$. Prior to incorporation into the surprise calculation, the agent modulates the MI by the reward history $(\bar{\delta})$. The logic is that, if the current set of internal agent states do not accurately correspond to the latent-states in the world, attention to all cues should increase. This modulation is accomplished through the equation,

$$
\begin{aligned}
& w_{A}=\left[1-\min \left(1, \min \left(0, \bar{\delta}-\xi_{D B, \text { floor }}\right) /\left(\xi_{D B, \text { ceil }}-\xi_{D B, \text { floor }}\right)\right)\right] * I_{M I} \\
& +\min \left(1, \min \left(0, \bar{\delta}-\xi_{D B, \text { floor }}\right) /\left(\xi_{D B, \text { ceil }}-\xi_{D B, \text { floor }}\right)\right),
\end{aligned}
$$

where the slope ranges from $\frac{1}{2}$ to 1 within the range of $\left(\xi_{D B, \text { floor }}, \xi_{D B, \text { ceil }}\right)$, and the intercept is restricted by $\left(\xi_{D B, \text { floor }}, \xi_{D B, \text { ceil }}\right)$. In all simulations, $\xi_{D B, \text { floor }}=-4$ and $\xi_{D B, \text { ceil }}=-12$.

\section{Distortion of discriminative attention}

To distort the use of discriminative attention we introduced a linear equation,

$$
w_{A} \leftarrow\left(1-\xi_{\text {Distortion }}\right) w_{A}+\xi_{\text {Distortion }}
$$

where $\xi_{\text {Distortion }}$ varied between zero and one.

\section{Final state estimation}

The discriminative attention weights are incorporated through the variable $w_{A}$ in equation 1 . If more than one internal state is less than the surprise threshold, the agent estimates a state through use of the softmax decision function,

$$
P\left(s(t) \mid P\left(c(t) \mid s_{i}\right)\right)=\exp \left(\beta_{S} P\left(c(t) \mid s_{i}\right) / \sum_{i} \exp \left(\beta_{\text {state }} P\left(c(t) \mid s_{i}\right),\right.\right.
$$

where $\beta_{S}$ governs the explore/exploit trade-off among states. After initial tuning, $\beta_{S}$ was fixed at 50 .

\section{Action selection and value updating}

Once the agent selects a state $s(t)$ for a given trial, the agent then uses a softmax function over action-values to select among actions:

$$
P(a \mid s(t))=\exp \left(\beta_{\text {Value }} Q(s(t), a)\right) / \sum_{a} \exp \left(\beta_{\text {value }} Q(s(t), a)\right) .
$$

The balance of explore/exploit among actions $Q(s(t), a)$ is dictated by $\beta_{\text {Value }}$ (fixed at 50). After selecting an action, the agent is reward $R(t)=1$, or the reward is omitted $R(t)=0$. The agent then calculates a reward prediction error (RPE) using the basic Rescorla-Wagner rule,

$$
\delta=R(t)-Q(s(t-1), a) .
$$

It updates the state action-value according to,

$$
Q(s(t), a)=Q(s(t-1), a)+\eta \delta .
$$




\section{Tracking reward history}

Agents track the running negative reward history using a fast timescale variable $\bar{\delta}_{\text {fast }}$ and a slow timescale variable $\bar{\delta}_{\text {slow }}$. This functionally enables an agent to distinguish between volatile environments and stable environments with low reward probabilities. Both values are computed using the equation,

$$
\bar{\delta}=\xi_{0} \bar{\delta}(t-1)+\xi_{1}\lceil\delta\rceil,
$$

where $\xi_{0}$ is a leak term, and $\lceil\delta\rceil$ is an integration term for the negatively rectified RPE. To compute $\bar{\delta}_{\text {fast }}$ and $\bar{\delta}_{\text {slow }}$, we first set a baseline timescale using $\xi_{0_{\text {baseline }}}=0.99$, and $\xi_{1_{\text {baseline }}}=1.50$. To shift the timescale while maintaining the asymptote, the agent uses a variable $\gamma$ implemented as,

$$
\begin{aligned}
& \xi_{0_{\text {shift }}}=\xi_{0}+\gamma \\
& \xi_{1_{\text {shift }}}=\xi_{1} *\left(1-\xi_{0_{\text {shift }}}\right) /\left(1-\xi_{0}\right) .
\end{aligned}
$$

An agent calculates the fast and slow timescales using, $\gamma$ fast $=-0.009$, and $\gamma_{\text {slow }}=0.004 . \bar{\delta}_{\text {effective }}$ is then determined trial-by-trial with,

$$
\bar{\delta}_{\text {effective }}=\left\lceil\bar{\delta}_{\text {fast }}-\bar{\delta}_{\text {slow }}\right\rceil .
$$

When a new state is created, $\bar{\delta}_{\text {fast }}$ and $\bar{\delta}_{\text {slow }}$ reset to zero.

\section{Updating of ProDAtt model states}

When a ProDAtt model agent is rewarded, it updates the prototype of selected state $s_{i}$ with the new example. If $s_{i}$ had been selected for fewer than $n$ trials, only the action-values $Q\left(s_{i}, a\right)$ are updated. After $n$ trials, the prototype vector $\mu_{i}$ is updated with a simple averaging of prior internal-state observations $j \in C_{i}$ (going back $n$ trials). The matrix $C_{i}$ contains only those trials classified as internal-state $s_{i}$ that were rewarded. The prototype cue vector $\mu_{i}$ is calculated with the equation,

$$
\mu_{i}=\frac{1}{n} \sum_{t-n}^{t} C_{i}(t) .
$$

Feature dimension prototype weights are determined by calculating the covariance of internal-state cue observations matrix $C$. This is done with the equation,

$$
\Sigma_{i}=\frac{1}{n-1} \sum_{t-n}^{t}\left(C_{i}(t)-\mu_{i}\right)\left(C_{i}(t)-\mu_{i}\right)^{\prime} .
$$

The precision matrix $I_{P, i}$ is then calculated from the covariance using,

$$
I_{P, i}=\Sigma_{i}^{-1},
$$

with a small amount of noise added drawn from $\mathcal{N}\left(0,10^{-8}\right)$ to aid in inversion.

\section{Updating of ExDAtt model states}

If an ExDAtt model agent is rewarded, the new example is simply added to the bank of known state examples.

\section{Example and trial count limits}

To ensure stability in the calculation of prototype mean and covariance or mutual information, we implement example and trial count minimums. For the mean and covariance, states must include at least 15 examples. Prior to the calculation of mutual information, the agent must have encountered at least 75 trials.

To allow the representations to evolve over the course of the session, and recognizing that memory does not have an infinite capacity, ProDAtt model agents included only the past 75 examples, while ExDAtt model agents included only the past 100 examples. 


\section{Random versus state estimation errors}

To investigate the differential patterns of random errors versus errors in state estimation, we introduced a probability of error in state selection on a given trial $(P($ error $)=0.3)$, and then varied the probability that error would induce random state selection versus selection of a similar state.

The ProDAtt model first calculated the Euclidean distance between state prototypes using the equation,

$$
d_{i j}=\sqrt{\sum^{k}\left(\mu_{i}-\mu_{j}\right)^{2}}
$$

In the ExDAtt model, the distance was calculated from every example in one state to every example in another state, and then averaged with a weight according to each example's frequency.

Once a vector $\vec{D}$ of distances was computed, the probability of selection was determined using the equation,

$$
P(s(t))=\exp \left(\beta_{\text {Error }} \vec{D}\right) / \sum_{i} \exp \left(\beta_{\text {Error }} \vec{D}\right)
$$

where a low value for $\beta_{\text {Error }}$ created random errors, while a high value of $\beta_{\text {Error }}$ biased errors towards the most similar other state.

\section{Artificial neural network actor-critic reinforcement learning}

We used an artificial neural network (ANN) to implement an actor-critic reinforcement learning model through the TensorFlow Keras package ${ }^{25,51}$. The input layer size corresponded to the length of the input vector, and then fed into a common layer of 124 ReLu units. An actor network received inputs from the common layer, and its size corresponded to the number of actions. The critic, composed of a single unit, also received common layer inputs. While the critic network's role is to predict the expected reward on a given trial, the actor network learns a value function that minimizes the difference between the critic's prediction and the experienced reward. That difference was computed using a Huber loss function, which then back-propagates through standard gradient decent with a learning rate of 0.01 .

To analyze the effect of variance in weight initialization, we followed the methods described in Flesch et al. ${ }^{52}$. We added noise to the each example, drawn from a $\mathcal{N}(0,0.1)$. We then initialized the weights on projections from the input layer to the hidden layer, with the weights drawn from a normal distribution centered on zero, and whose variance was specified as either $0.01,0.1,0.2,0.3,0.4,1.0,2.0$ or 3.0 .

\section{Experimental tasks}

We designed three tasks to test predictions from the algorithmic models. Stimuli used in the tasks varied along four dimensions: color, shape, texture and size. These stimuli were the examples used to infer latent rules. On a given trial, a subject might encounter an orange circle with dot texture of small size. On the next trial they might encounter a blue square with diagonal-lined texture of large size. Upon encountering a stimulus, they were then prompted to select an action. After selecting the action, they experienced an outcome (either a reward or no reward). Outcomes were dictated by the task's latent rules. Both the stimuli used and the latent rules depended the task (as described below). Each task was implemented both for computational models and human subjects.

\section{Single example and latent rule instrumental learning tasks}

To test the previous models' capacity for learning latent rules with stochastic rewards, we used simple instrumental learning tasks. In each version of the task, there were two states and two actions. The "single example instrumental learning task" involved one example per state (two total examples in the task) grouped according to color. The "latent rule instrumental learning task," on the other hand, involved four examples that could be grouped according to either color or shape, with the model needing to discover the appropriate latent rule. On a given trial, agents were presented the example, and then select an action. Reward was then provided or omitted according to the latent state. The single example and latent rule tasks were each then simulated using either deterministic rewards or stochastic rewards with $\mathrm{P}($ reward $)=0.8$. 


\section{Online task deployment}

Task implementation and recruitment We implemented the tasks as a webapp using the Django framework, and hosted them online through Microsoft Azure. We then recruited subjects through Amazon Mechanical Turk (mTurk) for the initial sample, or Prolific for the replication samples. This study was approved by the Yale Institutional Review Board (IRB). All participants were shown and accepted an IRBapproved online informed consent document and were paid for their participation. When they accepted, they were provided a link to our website, where they first entered their platform ID, and answered a few basic demographic questions (age, gender, education level). Those who completed the entire session were then asked to rate the difficulty of the task, to provide a verbal description of their strategy, and then chose a radio button option that best described their strategy.

For the Experiment 1 initial sample, 400 human subjects were recruited, 151 completed the tutorial, 141 completed session, 109 passed the performance threshold. For the Experiment 1 replication, 239 human subjects were recruited, 199 completed the tutorial, 197 completed session, and 156 passed the performance threshold. For Experiment 2, 100 were recruited, 62 passed the tutorial, 62 completed session, and 53 passed the performance threshold. For the Experiment 3 initial sample, 100 were recruited, 61 completed the tutorial, 59 completed the session and 49 passed the performance threshold. For the Experiment 3 replication, 123 were recruited, 109 completed the tutorial, 109 completed the session and 104 passed the performance threshold.

Prior to initiating the tutorial, subjects were asked to self-report basic demographic information. Demographic information for subjects who passed the performance threshold is given in Figs. S2 and S7.

Human subject compensation Human subjects received $\$ 2$ for completing the tutorial (whether passing or not), $\$ 4$ for completing the task and $\$ 4$ for achieving a performance level above $60 \%$.

Tutorial For all tasks, subjects first participated in a tutorial that instructed them on associating actions $(A$ and $B)$ with different latent rules. Actions during the tutorial were probabilistically rewarded. The first latent state was rewarded $90 \%$ for action $A$ and $0 \%$ for action $B$. The second latent state's rule was reversed, such that $A$ was rewarded $90 \%$ and $B 0 \%$. The third latent state had a mixed reward contingency, such that action $A$ was rewarded $70 \%$ and $B 30 \%$. After moving through several screens that interactively and explicitly taught them the reward contingencies, they were tested for 10 trials where examples from the latent states were randomly interleaved. After each trial, they received feedback both about reward, and that trial's action-reward probabilities. To move onto the main task, they had to select on 8 out of 10 trials the option with the largest reward probability. If they failed to pass on the first go, they were allowed to try a second time. If they failed the second time, they were given a payment token and sent back to the recruitment platform (mTurk or Prolific). The tutorial was implemented with this structure for three reasons: 1) instruct subjects on task structure; and 2) to screen out bots; 3) train subjects to expect stochastic rewards, as well as a multi-way mapping between actions and states.

General task structure Each experiment was framed as subjects being "space pirates," who had to learn which actions activate alien artifacts. On each trial, they were presented an example and attempted to activate it through actions associated with different key presses. Action-key associations were randomized across subjects. There was no time limit on their response. After responding, they were shown an outcome screen. If they chose the correct action, they were shown a glowing green energy crystal along with the following text:

Rewarded! The power was growing...

If they chose the incorrect action, they were shown an empty energy crystal, along with the following text:

Nothing happens. Bummer.

Every ten trials, subjects were shown a screen displaying how much energy they had accumulated, as well as how much was possible. At the completion of the tasks, subjects were provided a randomly generated payment token to enter on the recruitment platform. 


\section{Experiment 1: novel example generalization}

Human subjects and model agents (henceforth both referred by "subjects") were trained on example-action contingencies using examples composed of the following dimensional combinations: colors were orange, magenta or blue; shapes were circle, square or star; and texture was dots or diagonal lines. All examples were size large. There were four available actions: $A, B, C$ or $D$.

Sessions were composed of two blocks: initial learning, then generalization. During the initial learning block, subjects learned that all orange examples were rewarded for action $A$, examples shaped as stars were rewarded for action $B$, and circles for action $C$. Action $D$ was never rewarded. Examples during initial learning shared a texture (dots), which was noninformative. In the generalization block, novel examples were introduced identical to those of the first block, but differing from the initially learned examples in their texture (diagonal lines; full description in Table 2).

To be included in analysis, subjects had to have successfully completed the tutorial, the full task session, and exited the initial learning block with a minimum performance threshold of $50 \%$ correct. These criteria ensured we included subjects who had formed at least some effective state-representation of the task.

Human subject task implementation To facilitate learning, orange stars (which fit the rules for both action $A$ and action $B$ ) were excluded. During the initial learning block, there were 16 cycles of example presentation, resulting in 128 trials. Prior to beginning the session, subjects were provided the following message (where "A" was replaced by the key on the keyboard, etc.):

You stumbled upon a treasure trove of alien artifacts! To activate the alien artifacts, you might Shake (press A), Slap (press B), Kick (press C) or Bite (press D). It could be that all the actions were useful, or it could be that just a few were useful. There also might be patterns. You have to figure it out by trial and error. Good luck!

During the generalization block, there were 5 cycles, resulting in 80 trials. The transition from the initial learning block to generalization occurred without notice to subjects. Of note, for the replication study we changed the described actions to "sing," "dance," "wave" or "bite."

Model task implementation For the computational models, examples were composed of vectors where sections of the vectors were one-hot for a particular feature. For example, the portion of the vector for color might be $[1,0,0]$ for orange, $[0,1,0]$ for magenta, or $[0,0,1]$ for blue. The portion of the vector for shape might be $[1,0,0]$ for circle, $[0,1,0]$ for square, or $[0,0,1]$ for star. Thus, the vector for an orange star would be $[0,1,0,0,0,1]$. The sum of an example vector was always always the same within an experiment. For simulations involving the artificial neural network, noise was randomly added to stimulus vectors, drawn from the distribution $\mathcal{N}(0,0.1)$. Examples were presented semi-randomly in cycles. During a cycle, each potential example was presented a single time. Within cycles, example order was randomized.

For simulations, models were trained on 222 cycles for the initial learning block (with 9 examples, 1998 total trials), and 111 for the generalization block (18 examples, 1998 trials). This was standardized across model implementations. In figures showing learning curves, total session length was truncated.

\section{Novel context generalization tasks}

Subjects were trained sequentially in two separate contexts on latent rules, with each context involving distinct actions (actions $A$ and $B$ in context 1, actions $C$ and $D$ in context 2). The context were composed of four unique examples, and each action was rewarded for two unique examples. During the generalization context, examples from both prior contexts were all present.

Human subjects who reached a performance level of $50 \%$ correct on the final 10 trials of contexts 1 and 2 were included in analysis. This ensured they formed at least some effective representation of the states they could then use during generalization.

Version 1 (Experiment 2) Version 1 was designed to determine if subjects were using a discriminative model of state definition, and if so the type of model. 
Context 1 contained two examples rewarded for action $A$ that shared dimensions color (magenta), shape (circles) and texture (dots), but unique in size (large or small). Action $B$ examples in context 1 also shared those dimensions (orange squares with dot texture), and were also unique in size (large or small). Thus, the dimensions informative for actions in context 1 were color (magenta versus orange) and shape (circle versus square).

Context 2 was composed of examples rewarded for actions $C$ or $D$. Action $C$ examples differed in color (magenta and blue), but shared the dimensions for shape (circle), size (large) and texture (diagonal lines). Action $D$ examples differed from action $C$ examples in their shape (square). Thus, the dimension informative for determining actions in context 2 was shape (see Table 5 for full description).

Version 2 (Experiment 3) Version 2 was designed both to identify if subjects were utilizing a discriminative model, and to determine the degree to which behavior was shaped by prototype covariance, while also testing for the presence of a discriminative model. To accomplish this, the arrangement of features across latent states was designed to maximally differentiate model predictions.

Context 1 contained two examples rewarded for action $A$ that shared features shape (circles) and texture (dots), but differed in size (large or small) and color (magenta or orange). Action $B$ examples in context 1 shared shape (square), texture (dots) and size (large), but differed in color (magenta or orange). Thus, the dimension informative for actions in context 1 was shape (circle versus square).

Context 2 contained two examples rewarded for action $C$ that shared features shape (circles), size (large) and texture (diagonal lines), but differed in color (magenta or blue). Action $D$ examples in context 2 shared shape (square) and texture (diagonal lines), but differed in color (magenta or blue) size (small or large). Thus, the dimension informative for actions in context 2 was also shape (circle versus square; see Table 9 for full description).

Human subject implementation Subjects experienced 12 randomized example cycles in context 1 (48 total trials), 12 cycles in context 2 (48 total trials), and then 10 cycles during the generalization context (80 total trials). Prior to beginning block 1 (context 1), subject received the following instructions:

Welcome to Planet Waz-up, home to the long-deceased Waz civilization. Here you will find artifacts that were activated with either a shake (press A) or a slap (press B). You'll have to figure out what works!

Prior to context 2, they received the instructions:

Your work on Planet Waz-up was complete! After hopping in your spaceship, you traveled to Planet Oh-Kay. Here once lived the proud species Oh. Their artifacts operate completely differently, and were activated with either a kick (press C) or a bite (press D). Forget what you learned on Planet Waz-up. Planet Oh-Kay's artifacts have their own rules!

Prior to the generalization context, they received this instruction:

Good work space pirate! You learned of planet Blabla, the only place in the galaxy where both the Waz and Oh once lived! Here you discover artifacts from both civilizations. That means you can shake (press A), slap (press B), kick (press C), or bite (press D). Go collect some energy!

As with the novel example generalization task, during replication we changed the description of actions to "sing," "dance," "wave" or "bite."

Model task implementation The ProDAtt and ExDAtt models experienced 200 cycles ( 800 total trials) of contexts 1 and 2, then 50 cycles of the generalization context (400 total). The discriminative ANN experienced 250 cycles of contexts 1 and 2 (1000 trials each block), then 125 cycles of the generalization context (1000 total cycles). For all models, we masked selection of actions not available in a context (e.g. actions $A$ and $B$ were unavailable in context 2). 


\section{Behavioral analyses}

We conducted the following analyses of behavior during the experiments.

Learning curves We computed learning curves for subject performance in each experiment. To do so, on every trial we determined if a subject selected the most-rewarded action. If so, a "1" was recorded for that trial, otherwise a "0" was recorded. This provided a per-trial vector for each subject. To obtain population measures, we computed the mean and standard deviation of these vectors on a per-trial basis. To apply smoothing, we used a forward window of 5 trials.

\section{Experiment 1 (novel example generalization), specific analyses}

All analyses focused on the generalization block, when the novel examples joined the initially learned examples in the presentation cycle.

First generalization appearance difference (FGA) We examined the first appearance of each example during the generalization block and whether a subject selected the most-rewarded action. For each subject, we then separately averaged the performance on initially learned versus novel examples. The mean performance on initially learned examples was subtracted from the mean performance on novel examples. It can be summarized with the equation,

$$
\begin{aligned}
F G A= & P(\text { Correct } \mid \text { Novel Example, First Generalization Appearance })- \\
& P(\text { Correct } \mid \text { Learned Example, First Generalization Appearance })
\end{aligned}
$$

A more negative number indicates a worse performance on novel examples relative to initially learned examples, while zero indicates no difference.

Paired examples generalization difference (PGD) If subjects failed to fully generalize from initially learned examples to novel generalization examples, we expected an increased error rate for novel generalization examples. To assess this, we paired each novel example with the initially learned example that differed only in the noninformative dimension (texture). For example, two large magenta circles where the novel example had a striped texture and the initially learned example had a dots texture. For each member of the pair, calculated the error rate as the proportion of trials during the generalization block where the largest action was not chosen. The difference was taken as a simple subtraction of the initially learned example's error rate minus the novel example's error rate. This is captured by the equation,

$$
P G D=P(\text { Error } \mid \text { Novel Example })-P(\text { Error } \mid \text { Learned Example })
$$

To obtain a single measure for each subject, we averaged this value for a subject across all example pairs. A value of zero indicates no difference.

Exploration error (EE) During the initial learning block the other dimensions (color and shape) each uniquely formed states associated with specific actions $(A$ and $B)$. To assess exploration errors with novel examples, during the initial learning block action $D$ was never rewarded. If subjects questioned whether the novel generalization examples (with different texture) were distinct from the initially learned states, this could manifest as increased exploration of action $D$. We identified the first trial an example appeared during the generalization block, and whether the subject choose action $D$. It is described by the equation,

$$
E E=P(\text { Choose } D \mid \text { Novel Example, First Encounter })
$$

The value was averaged for each subject, with chance at 0.25 . Values above 0.25 indicate significant exploration of action $D$. 
Subpopulations Inspection of the histogram of FGA values for initial sample subjects indicated the potential for two distributions. The discrete nature of the values precluded the used of formal tests for bimodality. We thus visually set a threshold at -0.3 . We did not include the threshold value in the preregistration, though we did preregister a hypothesis for the existence of two distributions along the FGA.

\section{Experiments 2 and 3 (novel context generalization), specific analyses}

All analyses focused on the generalization context.

Initial generalization context error (IE) We identified the first trial an example appeared during the generalization context, and whether the subject choose the most-rewarded action.

$$
I E=P(\text { Error } \mid \text { First Generalization Appearance }) .
$$

That value was averaged for each subject. Given there were four potential actions, chance was 0.25 . A value above 0.25 indicates a significant number of errors during the first generalization encounter.

Discriminative errors (DE) If subjects preferentially attend to discriminative features, they will exhibit a specific error type we call a "discriminative error." This error occurs when, due to the exclusion of previously non-discriminative feature dimensions, distance between two perceptually distinct examples is zero. This measure assesses for the probability of a discriminative type error, given that the subject has made an error and is described by the equation,

$$
D E=P(\text { Discriminative Type|Error, First Generalization Appearance })
$$

The chance level is $33 \%$. Values significantly greater than $33 \%$ indicate preferential attention to discriminative features.

Initial generalization context errors for each context states (IEC) This metric quantifies the difference in errors between the latent states learned in each context. It is specified by the equation,

$$
\begin{aligned}
I E C= & \mid P\left(\text { Error } \mid \text { First Generalization Appearance, } \text { Context }_{1}\right)- \\
& P\left(\text { Error } \mid \text { First Generalization Appearance, } \text { Context }_{2}\right) \mid .
\end{aligned}
$$

Given there are only four stimuli each, we consider significant only differences greater than 0.5. A difference great than that value indicates they are generalizing differently between the latent states learned in each context.

Error rates throughout novel generalization context (NCE) We also computed the total rate of errors across the entire novel generalization context. This is a general measure of generalization performance.

$$
N C E=P(\text { Error } \mid \text { Generalization Context })
$$

Confusion matrices We created confusion matrices of each subject's errors. Rows in the subject's confusion matrix indicate the presented example, and columns indicate selected actions on error trials. When calculating the confusion matrix for individual subjects, this involved simply filling the matrix based on error actions. 
Feature bias (for novel context generalization task version 2) To calculate the feature bias, we exploited the fact the prototype feature distance of examples from the same initial learning context to another state in the other initial learning context will be equal, while the distance using all features will be unequal. Thus, a prototype model predicts that the proportion of errors will be the same for that action, while the a model where each example is memorized individually predicts that they will be significantly different.

To compute this, we first normalized the rows of each subject's confusion matrix by the total number of errors in that row. We then computed the difference in errors on the relevant action for each latent state's examples. For the examples of context 1 latent state action $A$, that meant the difference in the proportion of errors where they selected action $D$ (and vice versa). For the examples of context 1 latent state action $B$, that meant proportion of errors where they selected action $C$ (and vice versa). This difference was directional, such that the proportion of errors for the example with the further feature distance was subtracted from the proportion of errors for the example with the closer exemplar feature distance. Therefore, a positive value indicates a bias towards forming states for each example individually. The difference was then averaged for each subject across the four latent states. That produced a distribution of feature biases.

Subpopulations We separated subjects based on thresholds of the DE and IE. These thresholds were identified through visual inspection. In the novel context generalization task version 1 sample we used thresholds of $\mathrm{DE}=0.6$ and $\mathrm{IE}=0.4$. We identified the thresholds for the novel context generalization task version 2 using the initial sample subjects, and set them at $\mathrm{DE}=0.5$ and $\mathrm{IE}=0.4$. The version 2 thresholds were included in preregistration.

\section{Idealized attention models}

We formulated idealized models as to how subjects attend to features of an example, given they are using a specific model. Predictions were based on attention going into the generalization context, which is after all states had been learned, but before they were all encountered together. Attention was formulated differently for the discriminative models, the prototype covariance model, and the all-feature attention model. Using the attention predictions, we predicted the distances between examples presented on the screen, and the internal representations of examples (Fig. S9A and Fig. S16A). The distances is, by its nature, directional. We indicate this such that "presented example" refers to the example on the screen and "perceived example" refers to examples as they were represented perceptually by the subject.

Discriminative idealized attention models For the novel context generalization task version 1's two idealized discriminative attention models (first discriminative model and second discriminative model), we determined feature distance by applying the learned decision boundaries. The idealized discriminative attention model that learned only shape (first discriminative model) provided a relatively simple process. If examples were of different shape, their feature distance was 1 , otherwise it was 0 . Dimensions such as color, size or texture were irrelevant. The process was a bit more complicated for the idealized discriminative attention model that learned both color and shape in initial learning context 1, then only shape in initial learning context 2 (second discriminative model). To compute feature distance for this model, we used the decision boundaries that were formed during the initial learning context under question. For example, when the model considers the state membership of example 6 (large blue circle with diagonal texture), it considers the decision boundaries of the learning context under question (learning context 1). As the learning context 1 boundary involves two dimensions (color and shape), example 6 is a distance of 1 from action $A$, and two from action $B$. By applying these rules, we produced matrices of feature distance for both discriminative attention models. The feature distance matrix for the first discriminative model was symmetric, while the second discriminative model produces an asymmetric feature distance matrix.

Determining the discriminative idealized attention model for novel context generalization task version 2 was identical to the method use in determining the first discriminative model.

Prototype covariance idealized attention model The prototype covariance idealized attention model compares a presented example with an internal bank of prototypes. Thus, the feature distance depends on both the example presented, and the prototype to which it was being compared. Examples forming the state rewarded for action $A$ share color (magenta), shape (circle) and texture (dots), but differ in size (large and 
small). Thus, prototype precision weights (the inverse of prototype covariance) are high for color, shape and texture, but low for shape. Because of that, two examples have the same color (orange), shape (square) and texture (dots) but differ in shape (large and small) will have the same feature distance from the "action $A$ state." Working through each comparison of an incoming example and an example filtered by prototype covariance produced a feature distance matrix for the prototype covariance attention model. Due to the asymmetries between states in their covariance, the distance matrix was asymmetric.

All-feature idealized attention model Attention to all features means that every feature is used to compute the distance between presented and perceived examples. If two examples differ only in shape the distance will be 1 , while if they differ in both shape and color, the distance will be 2 . Given there is no filtering through reduced attention, the matrix all features idealized attention model feature distance matrix is symmetric.

Idealized attention model confusion matrices The predicted confusion matrices required two simplifying assumptions. First, as actions were associated with two examples, any error could reflect confusion with one of two examples whose feature distances from the presented example might be unequal. We therefore assumed that on error trials subjects confused the presented example based on the minimum feature distance to the chosen action. Second, we assumed that all errors go towards the state that is most similar to the true state.

\section{Bayesian regression}

We used Bayesian linear regression to fit the idealized model confusion matrices to observed confusion matrices. Each matrix was first flattened into a vector, and each predictor was assigned half-normal coefficient prior (Fig S10). There was also a half normal prior over the variance, and a full normal prior over the intercept. We used the Python package Bambi ${ }^{53}$ to fit the models through Markov chain Monte Carlo sampling. We assessed the quality of fits through each distribution's trace and $\hat{r}$ (Tables 7, 12 and 14), as well as by inspecting the posterior predictive distribution (Figs S11, S19, S20 and S29).

Model comparison To examine the impact of each idealized attention predictor, we dropped that predictor from the regression and assessed the quality of fit. We also fit a null model that included only the intercept and $\sigma$ distributions. Quality of fit was determined through use of Pareto smoothed importance sampling leave-one-out cross-validation ${ }^{30}$, as implemented by the Arviz Python package ${ }^{31}$.

Bayesian partial correlation coefficients We computed the partial correlation coefficients for each novel context generalization task version 1 predictor by multiplying the regression coefficient posterior distribution by its slope constant and then squaring that value. The full method is described in Appendix A.1 of Capretto et al. ${ }^{53}$.

Bayesian model recovery To assess the ability to recover idealized attention model predictions, we took each possible combination of models (e.g. prototype covariance alone, prototype covariance combined with random). We then fit the Bayesian model for 10 cycles, each time adding noise drawn from $\mathcal{N}(0,0.04)$. Recovering the novel context generalization task version 1, we assessed recovery through inspecting the coefficient distributions, model comparison and partial correlation coefficients (Fig. S12). Recovering the novel context generalization task version 2, we assessed recovery through inspecting the coefficient distributions and model comparison (Fig. S17).

Inferential statistics For all statistical tests, we used both frequentist and Bayesian statistics. Frequentist statistics were performed using the scipy package ${ }^{54}$. To compute Bayes factor, we used a combination of rpy2, and the R package BayesFactor ${ }^{55}$. 
Replication and preregistration After developing the analyses methods described, we conducted a preregistered replication of the results. That preregistration was done on June 16th, 2022, and can be found at OSF (osf.io/zc7hu) using the citation, "Pettine, W. W. (2021, May 18). Human latent state generalization through generative model learning with discriminative attention."

\section{ANN layer representations}

We analyzed representations in the hidden and action layers of the ANN during the period immediately prior to entering the novel context (block 3) of the novel context generalization tasks. To obtain the representations, we presented each example to the network and then stored the activation vectors from the hidden and action layers. For each layer, we then computed the Euclidean distance between representations using equation 23 .

Predicted representational dissimilarity matrices The predicted RDMs were computed by first creating states that would be learned by each idealized model (discriminative, prototype or exemplar) during the task. Thus, the states of a discriminative model would contain only to discriminative features, a prototype model would depend on the covariance of each state, and an exemplar model by the individual examples composing that state. To obtain the representations of each example, we then passed the examples through the states formed through each idealized model. For each of these idealized models, we then computed the euclidean distance between representations using equation 23 .

Fitting of representational dissimilarity matrices We first flattened the predicted and observed RDMs into vectors. We assigned beta weights to each prediction and then used ordinary least squares regression.

\section{Data and code availability}

- The simulation code is available at the repository: https://github.com/murraylab/instrumentalLatentStateLearning.

- The Jupyter notebook designed to aid in model intuition is available both through simulation repository, and hosted online through a link from its own repository:

https://github.com/murraylab/ProDAttExDAttModelIntuition.

- Task code is available at the repository: https://github.com/murraylab/alienArtifactsLearningTask.

- Upon publication, behavioral datasets will be released via a public dataset repository, and analysis code will be made available at https://github.com/murraylab/. 


\section{References}

[1] Gershman, S. J., Blei, D. M. \& Niv, Y. Context, learning, and extinction. Psychological Review 117, 197-209 (2010).

[2] Redish, A. D., Jensen, S., Johnson, A. \& Kurth-Nelson, Z. Reconciling reinforcement learning models with behavioral extinction and renewal: Implications for addiction, relapse, and problem gambling. Psychological Review 114, 784-805 (2007).

[3] Gershman, S. J. \& Niv, Y. Exploring a latent cause theory of classical conditioning. Learning $\&$ Behavior 40, 255-268 (2012).

[4] Collins, A. \& Koechlin, E. Reasoning, Learning, and Creativity: Frontal Lobe Function and Human Decision-Making. PLOS Biology 10, e1001293 (2012).

[5] Gershman, S. J., Monfils, M.-H., Norman, K. A. \& Niv, Y. The computational nature of memory modification. eLife 6, e23763 (2017).

[6] Cochran, A. L. \& Cisler, J. M. A flexible and generalizable model of online latent-state learning. PLOS Computational Biology 15, e1007331 (2019).

[7] Ashby, F. G. \& Maddox, W. T. Human Category Learning. Annual Review of Psychology 56, 149-178 (2005).

[8] Minda, J. P. \& David, S., J. Prototypes in category learning: The effects of category size, category structure, and stimulus complexity. Journal of Experimental Psychology: Learning, Memory, and Cognition 27, 775 (2001).

[9] Smith, D. J. \& Minda, J. P. Thirty categorization results in search of a model. Journal of Experimental Psychology: Learning, Memory, and Cognition 26, 3-27 (2000).

[10] Medin, D. L. \& Schaffer, M. M. Context theory of classification learning. Psychological Review 85, 207-238 (1978).

[11] Estes, W. K. Array models for category learning. Cognitive Psychology 18, 500-549 (1986).

[12] Nosofsky, R. M. Choice, similarity, and the context theory of classification. Journal of Experimental Psychology: Learning, Memory, and Cognition 10, 104-114 (1984).

[13] Nosofsky, R. M. Attention, similarity, and the identification-categorization relationship. Journal of experimental psychology: General 115, 39 (1986).

[14] Ashby, F. G. Multidimensional models of categorization. In Multidimensional models of perception and cognition, Scientific psychology series, 449-483 (Lawrence Erlbaum Associates, Inc, Hillsdale, NJ, US, 1992).

[15] Maddox, W. T. \& Ashby, F. G. Comparing decision bound and exemplar models of categorization. Perception \&3 Psychophysics 53, 49-70 (1993).

[16] Niv, Y. et al. Reinforcement Learning in Multidimensional Environments Relies on Attention Mechanisms. Journal of Neuroscience 35, 8145-8157 (2015).

[17] Love, B. C., Medin, D. L. \& Gureckis, T. M. SUSTAIN: a network model of category learning. Psychological Review 111, 309-332 (2004).

[18] French, R. M. Catastrophic forgetting in connectionist networks. Trends in Cognitive Sciences 3, 128-135 (1999).

[19] Kirkpatrick, J. et al. Overcoming catastrophic forgetting in neural networks. Proceedings of the National Academy of Sciences 114, 3521-3526 (2017). 
[20] Flesch, T., Balaguer, J., Dekker, R., Nili, H. \& Summerfield, C. Comparing continual task learning in minds and machines. Proceedings of the National Academy of Sciences 115, E10313-E10322 (2018).

[21] Parisi, G. I., Kemker, R., Part, J. L., Kanan, C. \& Wermter, S. Continual lifelong learning with neural networks: A review. Neural Networks 113, 54-71 (2019).

[22] Corbetta, M. \& Shulman, G. L. Control of goal-directed and stimulus-driven attention in the brain. Nature Reviews Neuroscience 3, 201-215 (2002).

[23] Noudoost, B., Chang, M. H., Steinmetz, N. A. \& Moore, T. Top-down control of visual attention. Current Opinion in Neurobiology 20, 183-190 (2010).

[24] Baluch, F. \& Itti, L. Mechanisms of top-down attention. Trends in Neurosciences 34, 210-224 (2011).

[25] Bhatnagar, S., Sutton, R., Ghavamzadeh, M. \& Lee, M. Natural Actor-Critic Algorithms. Automatica 45 (2009).

[26] Goodman, J. K., Cryder, C. E. \& Cheema, A. Data Collection in a Flat World: The Strengths and Weaknesses of Mechanical Turk Samples. Journal of Behavioral Decision Making 26, 213-224 (2013).

[27] Casler, K., Bickel, L. \& Hackett, E. Separate but equal? A comparison of participants and data gathered via Amazon's MTurk, social media, and face-to-face behavioral testing. Computers in Human Behavior 29, 2156-2160 (2013).

[28] Peer, E., Brandimarte, L., Samat, S. \& Acquisti, A. Beyond the Turk: Alternative platforms for crowdsourcing behavioral research. Journal of Experimental Social Psychology 70, 153-163 (2017).

[29] From lab-based to web-based behavioural research: Who you test is more important than how you test (2022).

[30] Vehtari, A., Gelman, A. \& Gabry, J. Practical Bayesian model evaluation using leave-one-out crossvalidation and WAIC. Statistics and Computing 27, 1413-1432 (2017).

[31] Kumar, R., Carroll, C., Hartikainen, A. \& Martin, O. ArviZ a unified library for exploratory analysis of Bayesian models in Python. Journal of Open Source Software 4, 1143 (2019).

[32] Mack, M. L., Love, B. C. \& Preston, A. R. Dynamic updating of hippocampal object representations reflects new conceptual knowledge. Proceedings of the National Academy of Sciences 113, 13203-13208 (2016).

[33] Mack, M. L., Preston, A. R. \& Love, B. C. Ventromedial prefrontal cortex compression during concept learning. Nature Communications 11, 46 (2020).

[34] Flesch, T., Nagy, D. G., Saxe, A. \& Summerfield, C. Modelling continual learning in humans with Hebbian context gating and exponentially decaying task signals. arXiv:2203.11560 [cs, q-bio] (2022).

[35] Heald, J. B., Lengyel, M. \& Wolpert, D. M. Contextual inference underlies the learning of sensorimotor repertoires. Nature 1-5 (2021).

[36] Miller, H. L., Ragozzino, M. E., Cook, E. H., Sweeney, J. A. \& Mosconi, M. W. Cognitive Set Shifting Deficits and Their Relationship to Repetitive Behaviors in Autism Spectrum Disorder. Journal of Autism and Developmental Disorders 45, 805-815 (2015).

[37] Lawson, R. P., Mathys, C. \& Rees, G. Adults with autism overestimate the volatility of the sensory environment. Nature Neuroscience 20, 1293-1299 (2017).

[38] Sevgi, M., Diaconescu, A. O., Henco, L., Tittgemeyer, M. \& Schilbach, L. Social Bayes: Using Bayesian Modeling to Study Autistic Trait-Related Differences in Social Cognition. Biological Psychiatry 87, 185-193 (2020). 
[39] Friedman-Hill, S. R. et al. What does distractibility in ADHD reveal about mechanisms for top-down attentional control? Cognition 115, 93-103 (2010).

[40] Kofler, M. J. et al. Working Memory Deficits and Social Problems in Children with ADHD. Journal of Abnormal Child Psychology 39, 805-817 (2011).

[41] Ramos, A. A., Hamdan, A. C. \& Machado, L. A meta-analysis on verbal working memory in children and adolescents with ADHD. The Clinical Neuropsychologist 34, 873-898 (2020).

[42] Taurines, R. et al. ADHD and autism: differential diagnosis or overlapping traits? A selective review. ADHD Attention Deficit and Hyperactivity Disorders 4, 115-139 (2012).

[43] Kern, J. K., Geier, D. A., Sykes, L. K., Geier, M. R. \& Deth, R. C. Are ASD and ADHD a Continuum? A Comparison of Pathophysiological Similarities Between the Disorders. Journal of Attention Disorders 19, 805-827 (2015).

[44] Antshel, K. M., Zhang-James, Y., Wagner, K. E., Ledesma, A. \& Faraone, S. V. An update on the comorbidity of ADHD and ASD: a focus on clinical management. Expert Review of Neurotherapeutics 16, 279-293 (2016).

[45] Collins, A. G. E. \& Frank, M. J. How much of reinforcement learning is working memory, not reinforcement learning? A behavioral, computational, and neurogenetic analysis. European Journal of Neuroscience 35, 1024-1035 (2012).

[46] Collins, A. G. E., Brown, J. K., Gold, J. M., Waltz, J. A. \& Frank, M. J. Working Memory Contributions to Reinforcement Learning Impairments in Schizophrenia. Journal of Neuroscience 34, 13747-13756 (2014).

[47] Collins, A. G. E., Ciullo, B., Frank, M. J. \& Badre, D. Working Memory Load Strengthens Reward Prediction Errors. Journal of Neuroscience 37, 4332-4342 (2017).

[48] Yoo, A. \& Collins, A. How Working Memory and Reinforcement Learning Are Intertwined: A Cognitive, Neural, and Computational Perspective. Journal of Cognitive Neuroscience 1-17 (2021).

[49] Friston, K. The free-energy principle: a unified brain theory? Nature Reviews Neuroscience 11, 127-138 (2010).

[50] Myung, I. J. Maximum Entropy Interpretation of Decision Bound and Context Models of Categorization. Journal of Mathematical Psychology 38, 335-365 (1994).

[51] Abadi, M. et al. TensorFlow: Large-Scale Machine Learning on Heterogeneous Distributed Systems. arXiv:1603.04467 [cs] (2016).

[52] Flesch, T., Juechems, K., Dumbalska, T., Saxe, A. \& Summerfield, C. Orthogonal representations for robust context-dependent task performance in brains and neural networks. Neuron (2022).

[53] Bambi: A simple interface for fitting Bayesian linear models in Python (2022).

[54] Virtanen, P. et al. SciPy 1.0: fundamental algorithms for scientific computing in Python. Nature Methods 17, 261-272 (2020). Number: 3 Publisher: Nature Publishing Group.

[55] Morey, R. D., Rouder, J. N., Jamil, T. \& Morey, M. R. D. Package 'bayesfactor'. URLh http://cran/rprojectorg/web/packages/BayesFactor/BayesFactor pdf i (accessed 1006 15) (2015).

[56] Barak, O., Rigotti, M. \& Fusi, S. The Sparseness of Mixed Selectivity Neurons Controls the Generalization-Discrimination Trade-Off. Journal of Neuroscience 33, 3844-3856 (2013).

[57] Bernardi, S. et al. The Geometry of Abstraction in the Hippocampus and Prefrontal Cortex. Cell 183, 954-967.e21 (2020).

[58] Multi-task representations in human cortex transform along a sensory-to-motor hierarchy (2021). 
[59] Ashby, F. G. \& Maddox, W. T. Relations between Prototype, Exemplar, and Decision Bound Models of Categorization. Journal of Mathematical Psychology 37, 372-400 (1993).

[60] Ashby, F. G. \& Gott, R. E. Decision rules in the perception and categorization of multidimensional stimuli. Journal of Experimental Psychology: Learning, Memory, and Cognition 14, 33-53 (1988).

[61] Reed, S. K. Pattern recognition and categorization. Cognitive Psychology 3, 382-407 (1972).

[62] Reed, S. K. \& Friedman, M. P. Perceptual vs conceptual categorization. Memory \& Cognition 1, 157-163 (1973).

[63] Nosofsky, R. M. Tests of an exemplar model for relating perceptual classification and recognition memory. Journal of experimental psychology: human perception and performance 17, 3-27 (1991).

[64] Smith, J. D., Murray, Morgan J. \& Minda, John Paul. Straight talk about linear separability. Journal of Experimental Psychology: Learning, Memory, and Cognition 23, 659 (1997).

[65] Smith, J. D. Prototypes in the mist: The early epochs of category learning. Journal of Experimental Psychology: Learning, Memory, and Cognition 24, 1411 (1998).

[66] Kruschke, J. K. ALCOVE: an exemplar-based connectionist model of category learning. Psychological Review 99, 22-44 (1992).

[67] Kruschke, J. K. \& Erickson, M. A. Learning of rules that have high-frequency exceptions: New empirical data and a hybrid connectionist model. In Proceedings of the Sixteenth Annual Conference of the Cognitive Science Society (Routledge, 1994).

[68] Kruschke, J. K. Base rates in category learning. Journal of Experimental Psychology: Learning, Memory, and Cognition 22, 3-26 (1996).

[69] Kruschke, J. K. Toward a Unified Model of Attention in Associative Learning. Journal of Mathematical Psychology 45, 812-863 (2001).

[70] Kruschke, J. K. \& Johansen, M. K. A model of probabilistic category learning. Journal of Experimental Psychology. Learning, Memory, and Cognition 25, 1083-1119 (1999).

[71] Nosofsky, R. M., Palmeri, T. J. \& McKinley, S. C. Rule-plus-exception model of classification learning. Psychological Review 101, 53-79 (1994). 


\section{Main Figures}

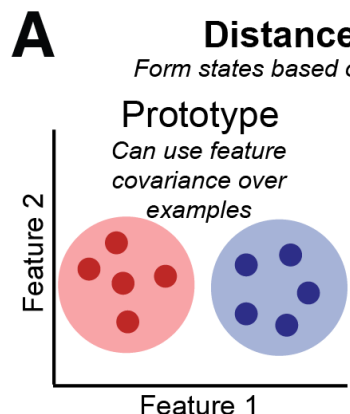

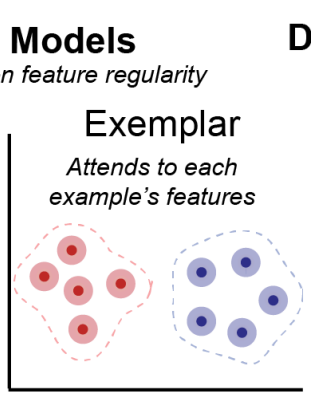

Feature 1
Discriminative Model B

Forms States Based On

Differing Features

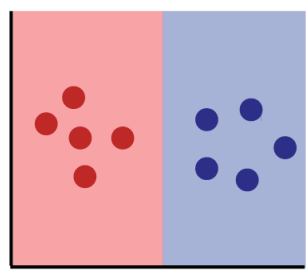

C

Distance Models Failure (Novel Example Generalization)

Initial Learning
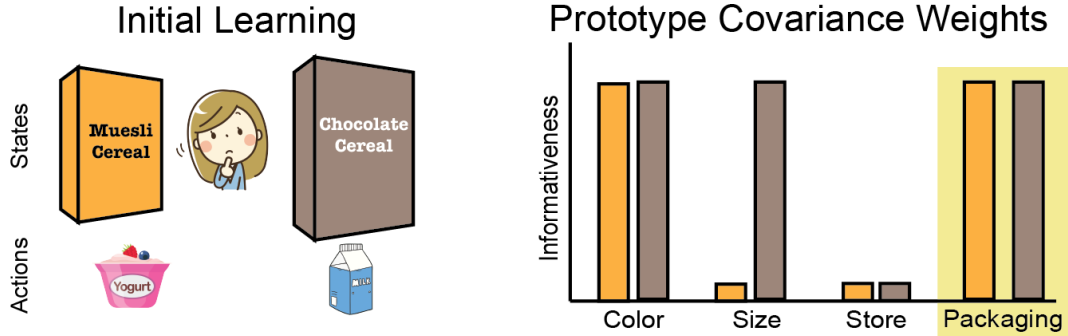

Generalization Failure

D

\section{Discriminativ}
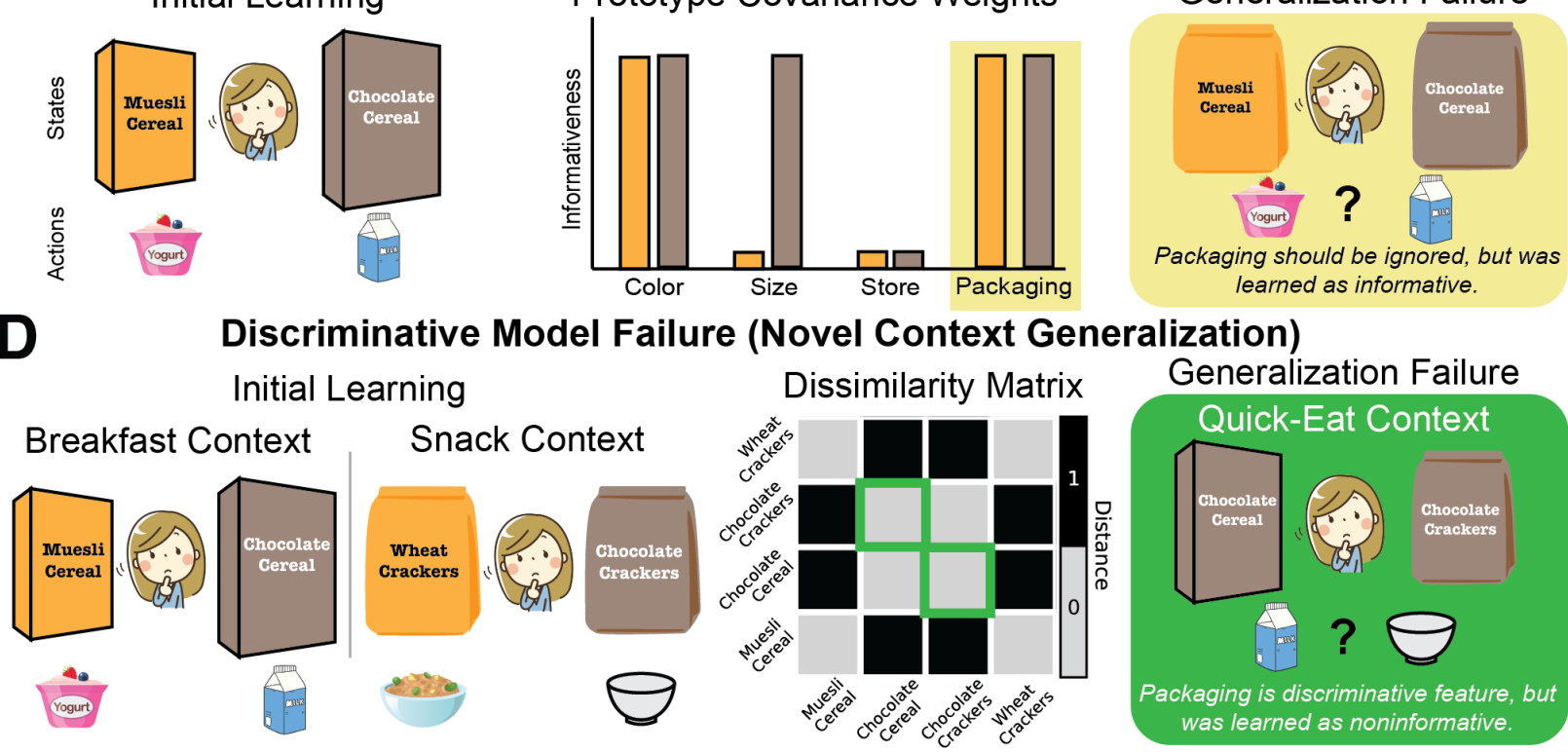

Figure 1: Distance and discriminative models of latent-state learning produce distinct generalization failures. (A) Prototype and exemplar models rely on a distance metric to determine state membership. This produces states whose weights on individual feature dimensions (shape of the cluster) do not depend on their informativeness for comparative decisions. A discriminative model (right) defines states based on feature dimensions that are informative for separating one state from another. This produces state boundaries defined by those dimensions most informative for discrimination. (B) Common grocery store items can be considered latent states of which one encounters only variable examples. Each item is associated with sets of actions. (C) A distance model can fail to generalize when a highly regular, yet noninformative feature changes in novel examples. During initial learning, the packaging is highly regular across examples (left), and thus it is considered informative (middle). When this feature is altered in novel examples, a distance model struggles to generalize the rewarded action (right). (D) A purely discriminative model can fail to generalize when previously noninformative features become informative in a novel context. During learning in the initial contexts, packaging was non-discriminative (left). Thus, the distance is zero between otherwise-identical alternatives learned in separate contexts (middle). This leads to generalization failure when these alternatives are brought together in a novel context (right). 


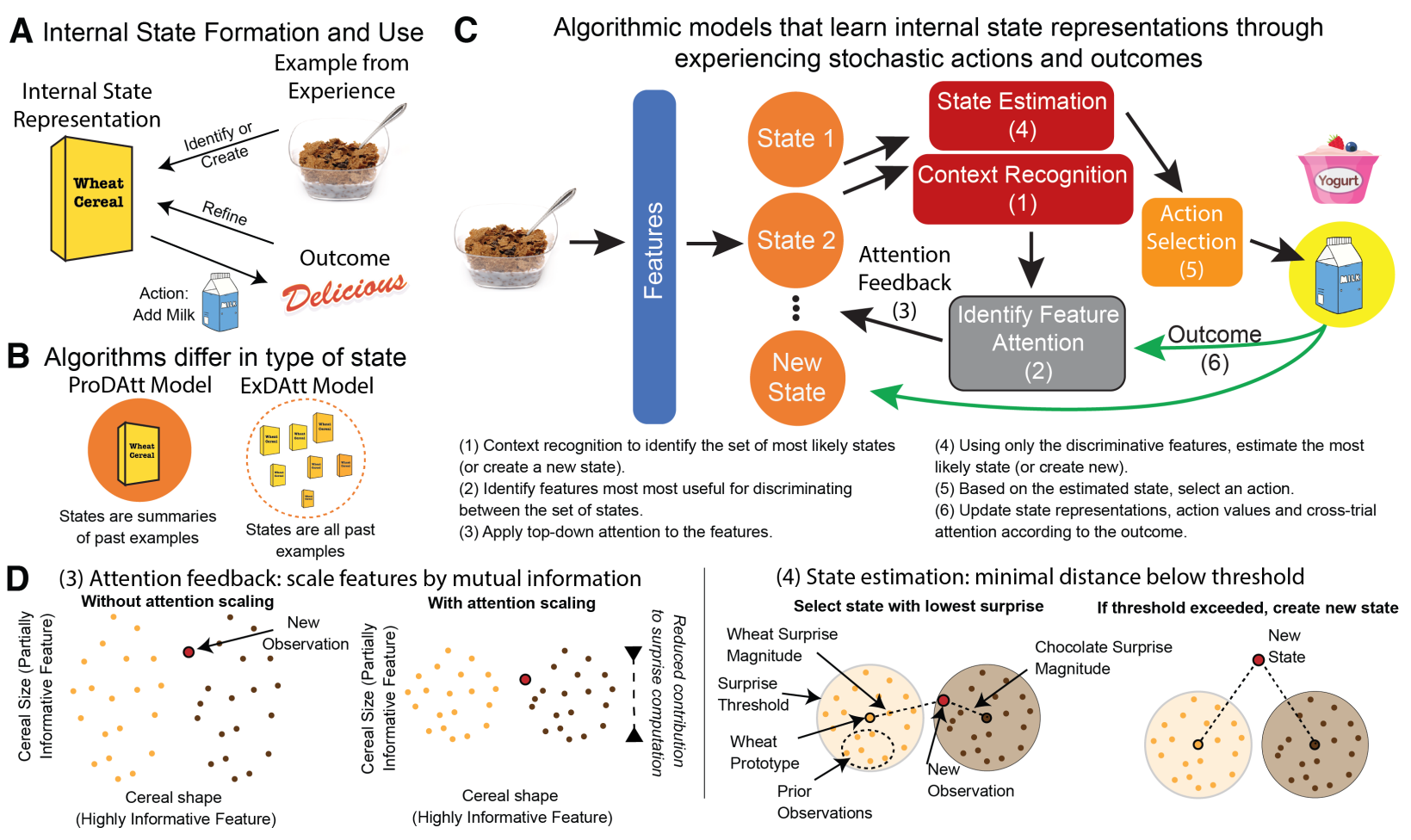

Figure 2: Algorithmic models of latent-state instrumental reinforcement learning using prototype or exemplar with discriminative attention. (A) Depending on feedback, novel examples either refine the representation of existing states, or drive the creation of new states. (B) In the "prototype states with discriminative attention" (ProDAtt) model, states are defined by the mean and covariance of past examples, while in the "exemplar states with discriminative attention" (ExDAtt) model, every past state examplar is used. (C) On a given trial, a ProDAtt or ExDAtt model agent encounters an example vector of features that it maps to the known bank of states. 1) The agent then uses a threshold to recognize its current context. The threshold is based on a Bayesian surprise metric that determines the distance from the example vector to each known state (less-surprising means closer). We use surprise, as it is a well-described transformation of the posterior probability in psychology and neuroscience setting $^{49}$. If no state is less-surprising than a threshold, the agent identifies the stimulus as a new state. If more than one state is below the threshold, the agent includes those states in the context. 2) The agent then calculates the mutual information of each feature for its usefulness in differentiating between states. By comparing the entropy of a feature within each state to its entropy across states, the mutual information identifies feature dimensions that maximally discriminate between states in a context. For each feature, that mutual information value determines its discriminative attention weights. The attention weights are modulated by the integrated reward history, such that heightened reward volatility increases overall attention. 3) These attention weights provide feedback to scale the feature values of each state. 4) The agent then uses these attention-scaled states in state estimation, and recalculates the surprise metric. If multiple states are below a second surprise threshold, the agent selects the least surprising (i.e. closest). If no state is below the second surprise threshold, the agent creates a new state. Each state learns a set of values for the available actions in the task. 5) Once a state has been selected, that state's values are probabilistically used to choose an action. 6) The agent uses that outcome to update the state representation and the action value. It also tracks the reward history. (D) The attention feedback step uses mutual information to scale feature dimensions by their discriminative informativeness (left). Either the most proximal state is selected, or if the distance is greater than threshold the agent creates a new state (right, ProDAtt model example). 


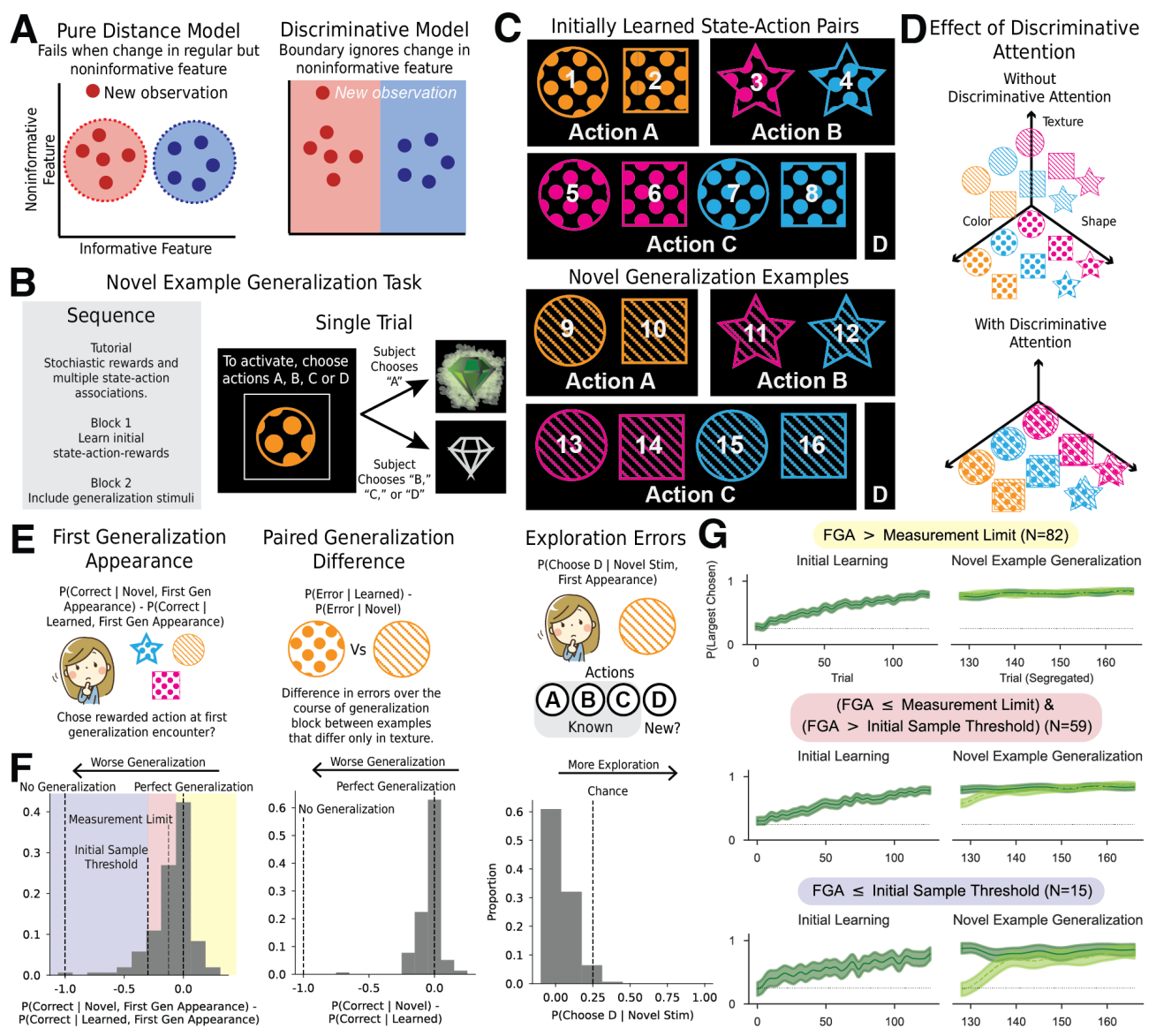

Figure 3: Experiment 1 - Human subjects use discriminative attention when generalizing to novel examples. (A) A model using distance to define state membership can fail to generalize if a new observation differs in a highly regular, but noninformative feature (left). However, a model using discriminative boundaries easily generalizes in this scenario (right). (B) Subjects learn the latent states defining action-reward associations. During the tutorial humans are instructed on stochastic rewards, that a single action can garnish rewards for multiple states, and that a single state can be rewarded for multiple actions. On a given trial, they encounter an "alien artifact" activated with one of four actions. The main task is composed of two blocks. During block 1, they learn the latent states with an initial set of examples. The transition to the second block occurs without notice to subject. During block 2, new examples are introduced that differ in a previously non-discriminative feature (left). After selecting an action, they are provided feedback with the outcome. (C) Latent-state exemplars, grouped by rewarded action. Initially learned examples are on the top (present block 1 and 2), and the novel generalization examples on the bottom (introduced in block 2). Action $D$ is never rewarded. (D) The feature space for novel examples is reshaped by discriminative attention. Without discriminative attention (top), novel examples are separated from the initially learned examples by their texture. With attention scaling (bottom) novel examples are projected into the same feature space as the initially learned examples. (E) During the generalization block, differences in performance on the first appearance of initially learned versus novel examples can measure generalization (left). The difference in error rates over the course of a session for discriminatively-identical paired examples can also quantify generalization (middle). When first encountering a novel example, the choice of action $D$ for novel example indicates a weak prior over state membership (right). (F) The first generalization appearance metric reveals that most subjects generalize to new examples, while a tail in the distribution shows individual variation (left). Paired generalization difference shows similar results (middle). Exploration errors are below chance for the majority of subjects (right). (G) Learning curves during initial state formation are similar across subjects with different first generalization appearance scores, but diverge during generalization. Those with a first generalization appearance score within the measurement error of zero show no difference in generalization learning curves between initially and novel examples (top). Those who are between the measurement error and the threshold found in initial sample subjects show moderate divergence during generalization (middle). Those who below the initial sample threshold show larger divergence (bottom). The shaded areas represent the SEM. 

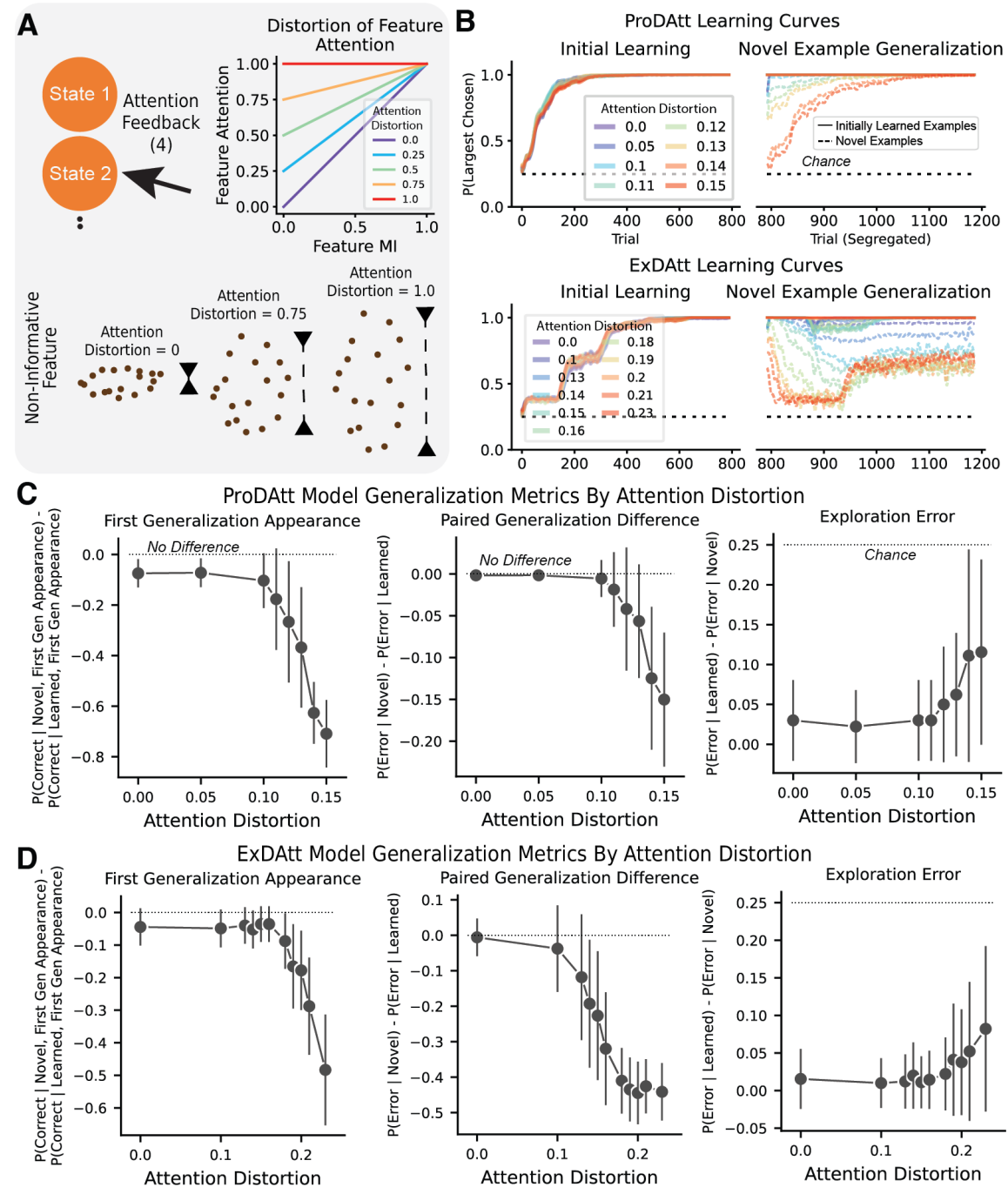

Figure 4: Experiment 1 - Varying algorithmic model use of top-down discriminative attention recapitulates the spectrum of human behavior. (A) In the ProDAtt and ExDAtt models, increasing the attention distortion parameter reduce sensitivity to attention feedback. This, in turn, increases the impact of variance in nondiscriminative features. (B) Learning curves from the ProDAtt model (top) and ExDAtt model (bottom) reveal that increasing attention distortion has no effect on initial learning, but separates the trajectory of initially learned and novel examples during generalization. For visualization, only the mean values are shown. (C) Distorting attention in the ProDAtt model causes the magnitude of the first generalization appearance difference to increase (left), along with that of the paired generalization difference (middle). The proportion of exploration errors, however, remain below chance (right). (D) The ExDAtt model shows the same trends. Error bars represent the standard deviation. 

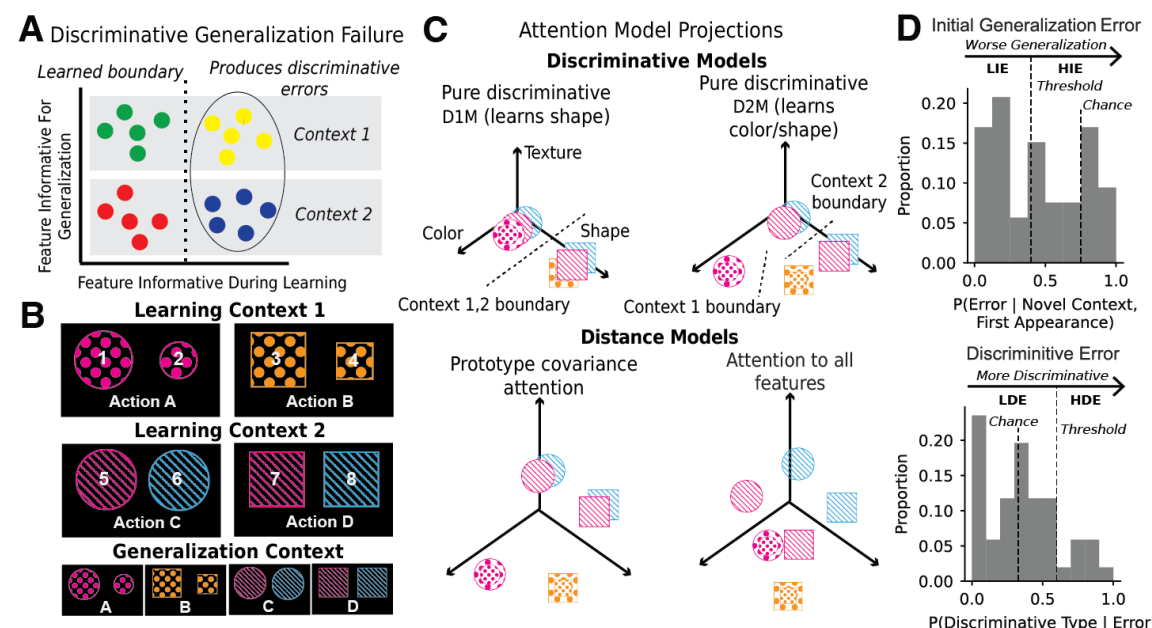
P(Error | Novel Context,
First Appearance) Discriminitive Error

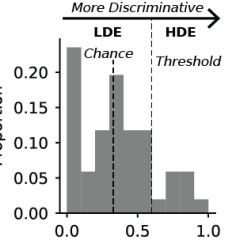

E

Idealized Attention Model Confusion Matrices F Subject Confusion Matrices
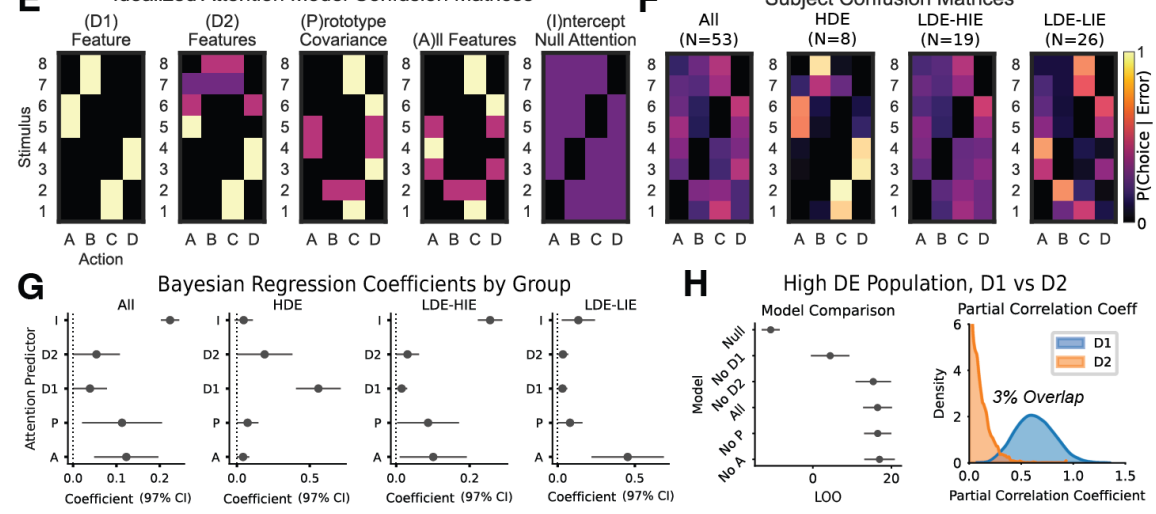

Bayesian Regression Coefficients by Group

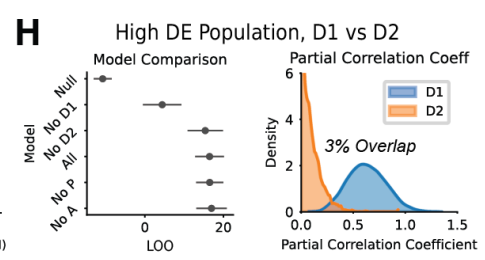

Figure 5: Experiment 2- Majority of subjects use previously non-discriminative feature to generalize in novel context. A subpopulation attends to one discriminative feature. Discriminative model with one feature (D1M), discriminative model with two features (D2M), intercept (I), prototype covariance (P), all-feature attention (A), all subjects (All), high discriminative error (HDE), low discriminative error (LDE), high initial error (HIE), low initial error (LIE), (A) When a purely discriminative model learns states, it defines states according to features that are maximally informative for decisions. It can fail to generalize when a feature non-discriminative during initial learning becomes the key discriminative feature for later decisions. This produces a distinct "discriminative error," where the key feature dimension separating states in earlier contexts was initially learned as noninformative. (B) The novel context generalization task version $1\left(\mathrm{CG}_{1}\right)$, where subjects learn distinct state-action contingencies sequentially in two explicitly signaled contexts before entering a third explicitly signaled generalization context where all latent states are active. Examples vary along the dimensions color, shape, texture and size. During a tutorial, subjects are taught to expect stochastic rewards, as well as a multi-way mapping between states and actions. (C) A pure discriminative model that learns only shape (D1M) will form a single decision boundary that fails to distinguish states falling on the same side of the decision boundary (top left). A pure discriminative model that learns the informativeness of color and shape in the first block will form a decision boundary for initial context 1 states involving color and shape, and a separate decision boundary for initial context 2 states involving only shape (D2M, top right). A model that uses the prototype covariance regularity of individual state features, resulting in distinct states (bottom left). If all features are attended equally, they will each occupy an independent location in feature space (bottom right). For visualization purposes, the size dimension is not shown and stimuli are slightly offset. (D) Human subjects vary in the probability of initial generalization errors (top) and discriminative errors (bottom). These metrics were used as thresholds for subpopulations. Initial generalization errors quantify the probability that subjects select the rewarded action the first time they encounter each example in the generalization context. Discriminative errors quantify the proportion of errors over a trial where subjects chose an action from the other context that differed along the non-discriminative feature (see panel A). (E) The idealized attention models produce predictions for distributions of errors visualized as confusion matrices. (F) The subpopulations of subjects produce distinct confusion matrices. (G) Fitting subjects' observed confusion matrices by the idealized attention models reveals distinct patterns in the coefficient weights. Shown are the mean and the $97 \%$ CI. (H) Both the model comparison (left) and partial correlation coefficients (right) reveal that subjects above the discriminative error threshold are better fit by the D1M relative to the D2M. The model comparison shows the mean and SE for Pareto smoothed importance sampling leave-one-out cross-validation. 


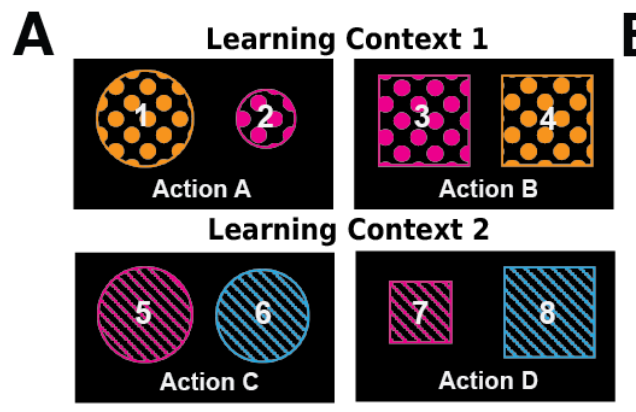

Generalization Context

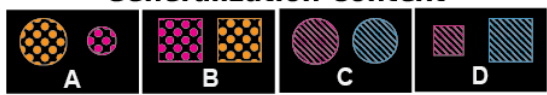

C Idealized Attention Model Confusion Matrices

(D)iscriminative (P)rototype

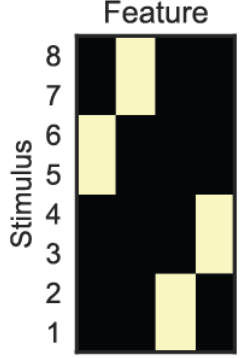

A B C D

Action

D

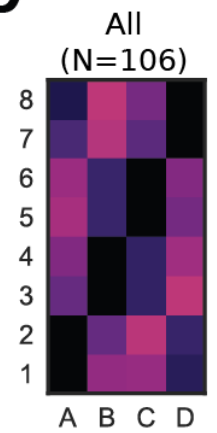

Covariance

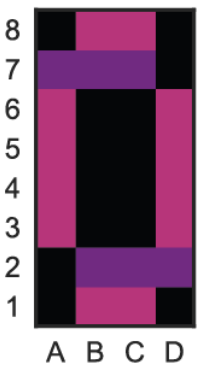

A B C D

Subject Confusion Matrices

HDE

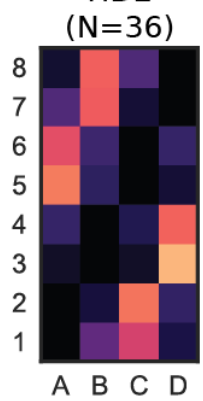

(A)II Features

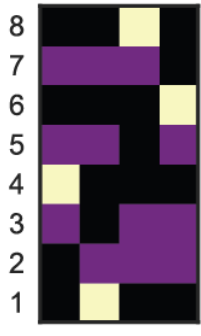

A B C D

LDE-HIE

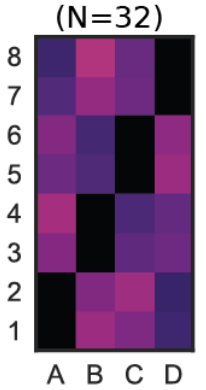

(I)ntercept Null Attention

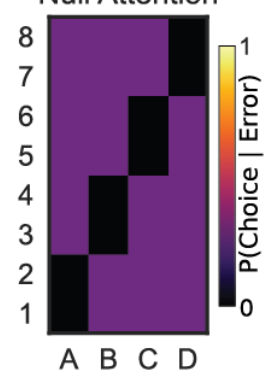

A B C D

\section{LDE-LIE}

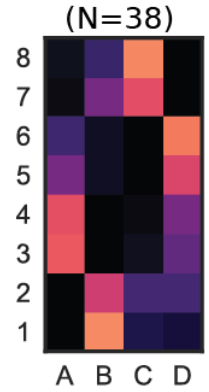

Discriminitive Error
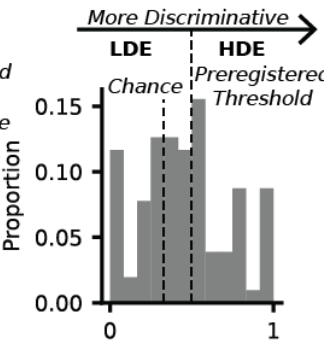

P(Discriminative Type |

Error)

E Bayesian Regression Coefficient Distributions

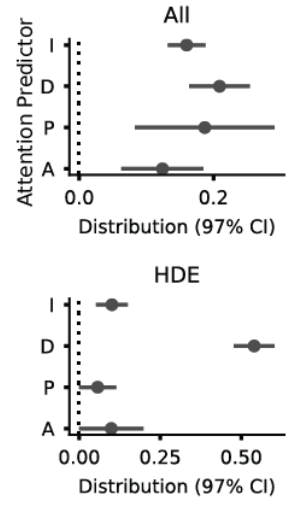

LDE-HIE

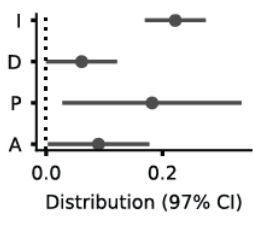

LDE-LIE

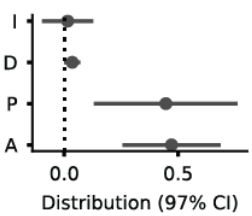

Figure 6: Experiment 3 - Majority of subjects attend to common state features. (A) The novel context generalization task version $2\left(\mathrm{CG}_{2}\right)$ produces greater separation between the idealize prototype covariance attention model and the idealized attention to all features model. It still detects discriminative errors. (B) Preregistered replication subjects on the $\mathrm{CG}_{2}$ task also vary in their generalization metrics, are divided into subpopulations using preregistered thresholds. (C) Each idealized attention model makes distinct predictions for $\mathrm{CG}_{2}$ error patterns. (D) Subpopulations of replication subjects on the $\mathrm{CG}_{2}$ task produce confusion matrices with distinct patterns. Use of the prototype covariance attention model indicates the formation of states incorporating multiple examples, while exclusively using the all-feature attention model indicates separate states for each example. (E) All subjects pooled together are ambiguous in their attention model (top). Subjects above the discriminative error threshold (HDE) have the highest regression coefficient for the discriminative attention model (mid-top). Subjects below the discriminative error threshold (LDE) have high weights on prototype covariance (mid-bottom and bottom). LDE subjects above the initial generalization error threshold (LDE-HIE) highly random errors (mid-bottom). Subjects below both thresholds (LDE-LIE) have high weight on the prototype covariance and all-feature attention models (bottom). Shown are the mean and SEM. This confirmed preregistered hypotheses. 

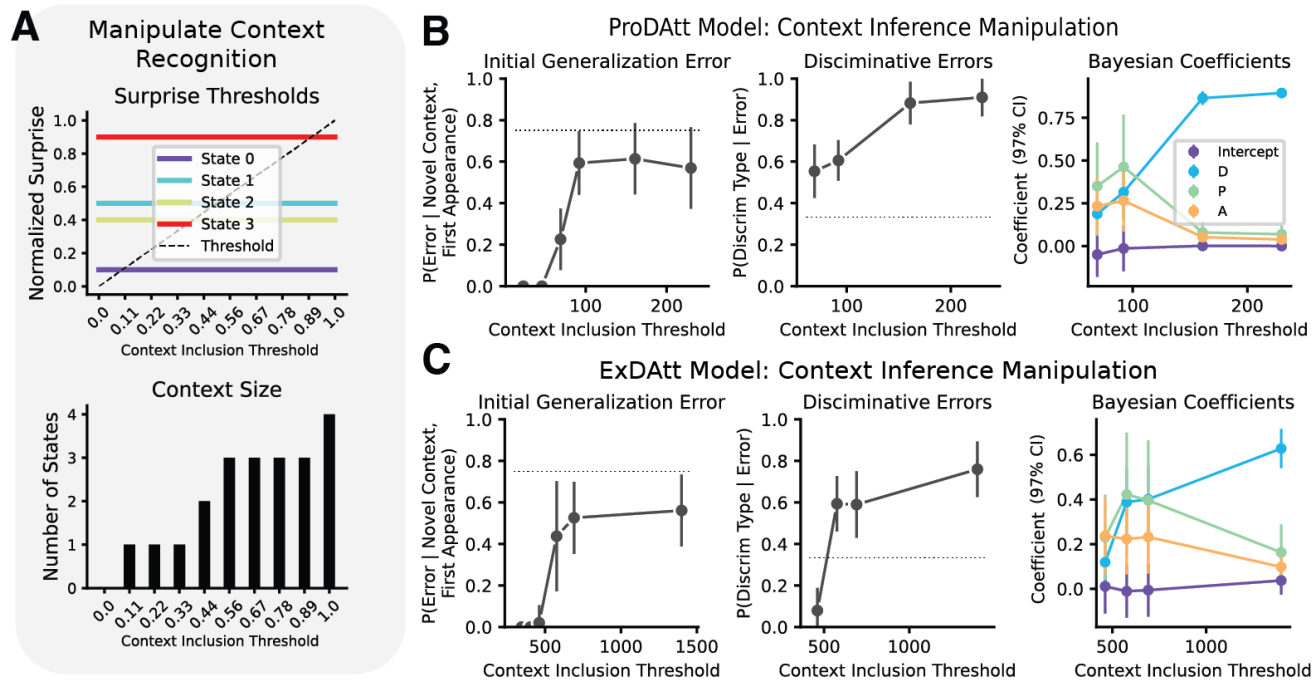

Figure 7: Reproducing discriminative feature attention subpopulation through alteration of algorithmic model context recognition, or artificial neural network hidden layer weights. (A) Increasing the context recognition surprise threshold (top) includes more states in the context (bottom). (B) In the ProDAtt model, A higher surprise threshold increases the initial generalization errors (left) as well as discriminative errors (middle), and shifts the coefficient values toward a discriminative model (right). (C) The same occurs with the ExDAtt model. Error bars for the error types indicate the standard deviation. Error bars for the Bayesian coefficients represent their $97 \%$ CI. 

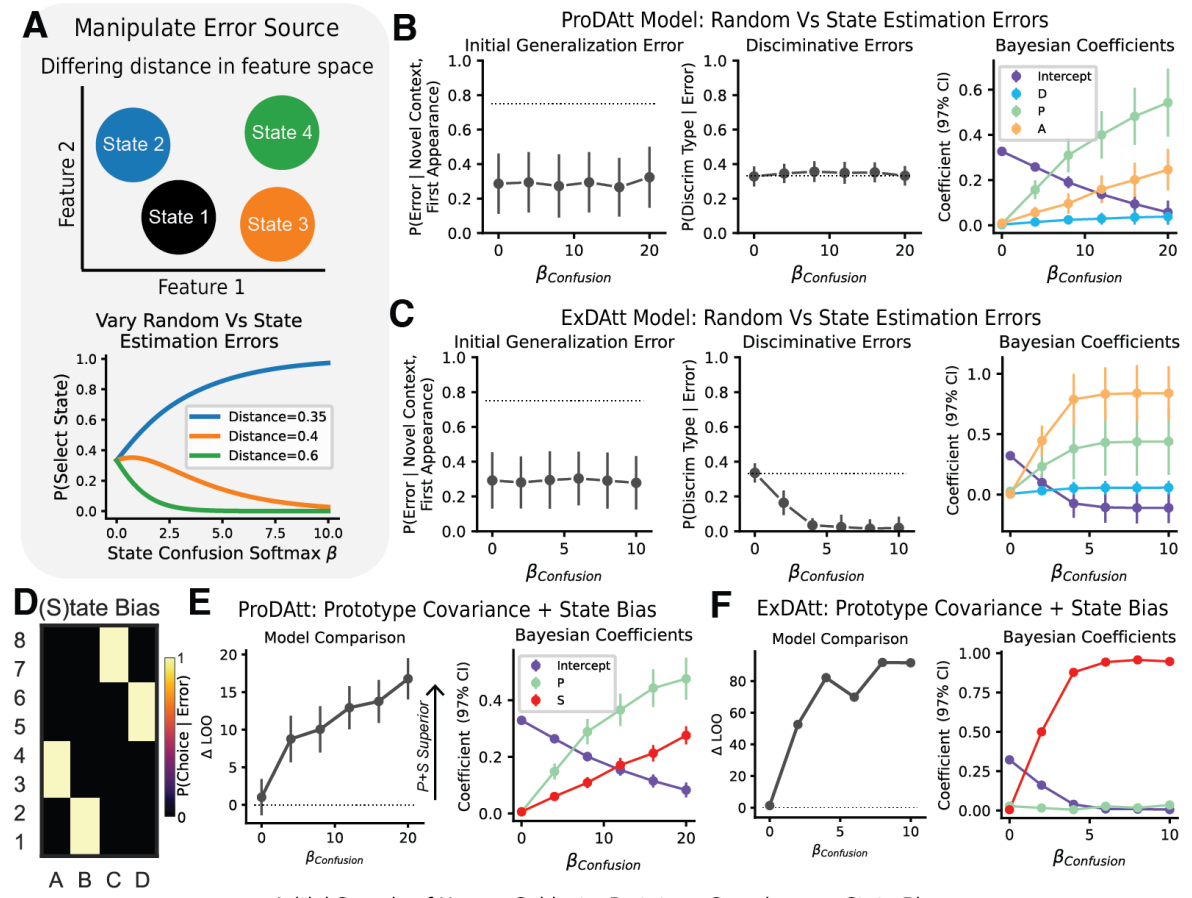

F ExDAtt: Prototype Covariance + State Bias
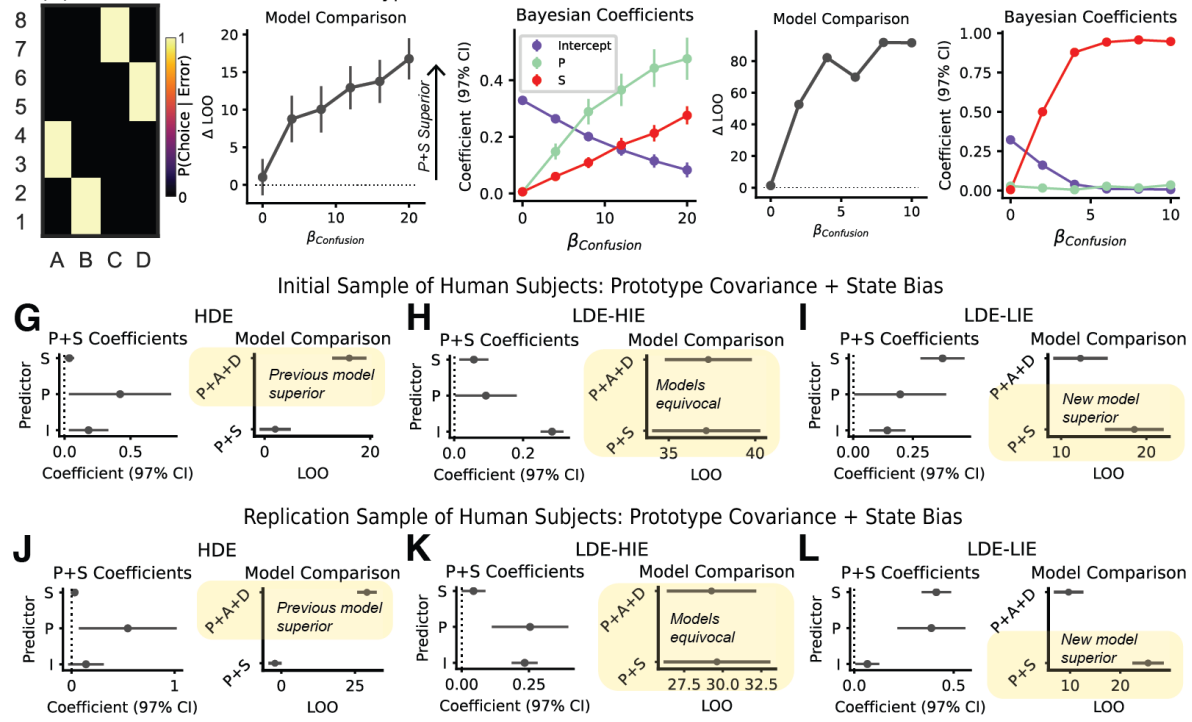

State Bias
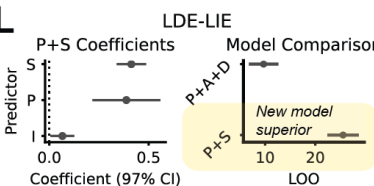

Figure 8: The majority of human subjects and the ProDAtt model attend to prototype covariance. (A) The manipulation of errors from random to the most proximal state in the algorithmic models. Low values of the $\beta$ parameter for state confusion errors resulted in random errors, while high values biased the errors towards states that were closer in distance. (B) In the ProDAtt model, increasing the state confusion softmax $\beta$ has no effect on initial generalization (left) or discriminative errors (middle), but increases the mean of the prototype covariance distribution (right). (C) In the ExDAtt model, the state confusion softmax $\beta$ has no effect on initial generalization errors (left), but does affect discriminative errors (middle). It also causes the coefficient values to favor the model that attends to all features (right). (D) The confusion matrix for an idealized attention model where errors are biased by other state exemplars (either the prototype mean or pooled exemplar distances). (E) As state confusion softmax $\beta$ increases, fitting the ProDAtt model confusion matrix with the prototype covariance and state bias attention models results in a better cross validation (positive $\delta \mathrm{LOO}$ ) for the $\mathrm{P}+\mathrm{S}$ regression model relative to the $\mathrm{P}+\mathrm{A}+\mathrm{D}$ regression model (left). Increasing state confusion softmax $\beta$ results in an increase for the prototype covariance and state bias coefficients, as well as a decrease of the intercept (right). (F) The ExDAtt model the $\mathrm{P}+\mathrm{S}$ regression also improves relative to the $\mathrm{P}+\mathrm{A}+\mathrm{D}$ regression (left). ExDAtt model regression coefficient increases only for the state bias idealized attention model, while the prototype covariance remains at zero, and the intercept decreases (right). (G/J) Initial sample and replication subjects above the discriminative error threshold are best fit by the model that includes the idealized discriminative attention model. (H/K) Initial sample and replication subjects below the discriminative error threshold and above the initial error threshold have significant prototype covariance coefficients, but are ambiguous in their fit by either regression model. (I/L) Initial sample and replication subjects below the discriminative error threshold and below the initial error threshold have a high coefficient for prototype covariance and are best fit by the $\mathrm{P}+\mathrm{S}$ model. Error bars for the error types indicate the standard deviation. Error bars for the Bayesian coefficients represent their $97 \%$ CI. The LOO is shown for its mean value and SE. 


\section{Appendix A: Additional preregistered analyses}

We preregistered several additional analyses that, for completeness, are reported here.

\section{Novel context generalization task version 1: early generalization versus late}

In our initial sample, there were indications that subjects errors arose from different forms of attention over the course of the generalization block. However, the subject sample number was too low for us to draw any conclusions. We therefore preregistered a hypothesis that subjects below the discriminative error threshold, there would be a shift in attention models over the course of generalization. That is, we hypothesized that errors in the second half of the generalization block would show more attention to prototype covariance. We found instead that, in both subpopulations, there was an increase in the coefficient for all-feature attention (Fig. S30, 18).

\section{Novel context generalization tasks: errors over the course of generalization}

For each of the four groupings by performance metric thresholds, we quantified proportion of error trials during the generalization block $\left(\mathrm{CG}_{1}\right.$ in Fig. $\mathrm{S} 31 \mathrm{~A}$, initial $\mathrm{CG}_{1}$ sample in Fig. S32A, replication $\mathrm{CG}_{1}$ sample in Fig. S32C). We found all groups produced errors at a proportion significantly greater than zero. Full statistical results are summarized in Table 19).

\section{Novel context generalization tasks: difference in performance during generaliza- tion between states.}

We considered whether there could be a performance difference during generalization between examples encountered during learning context 1 versus examples encountered during learning context 2. For each subject in each of the four groupings, we averaged first-encounter performance among examples learned in the same context. We then subtract the mean performance of learning context 2 from those of learning context 1 , and pool across all subjects within a group $\left(\mathrm{CG}_{1}\right.$ in Fig. $\mathrm{S} 31 \mathrm{~B}$, initial $\mathrm{CG}_{1}$ sample in Fig. $\mathrm{S} 32 \mathrm{~B}$, replication $\mathrm{CG}_{1}$ sample in Fig. S32C). In no group was there a significant difference in generalization performance between examples for the different learning contexts (Table 20). 


\section{Appendix B: Algorithmic notation}

Here we include formal algorithmic notation for the ProDAtt and ExDAtt models.

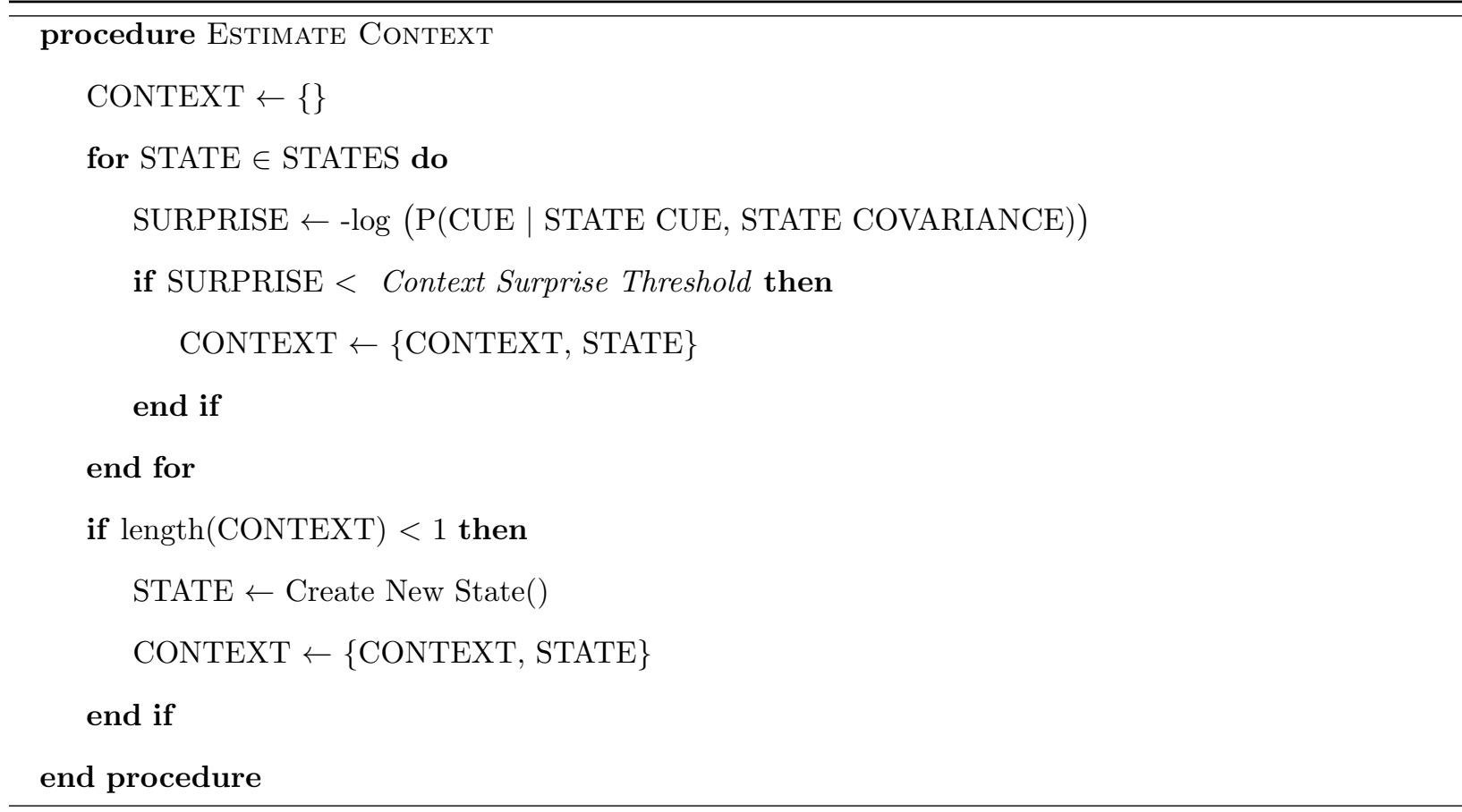

procedure Compute Feature Attention (Mutual Information)

CUE WEIGHTS $\leftarrow\{\}$

for FEATURE in CUE do

FEATURE STATE ENTROPIES $\leftarrow\{\}$

for STATE $\in$ CONTEXT do

FEATURE STATE ENTROPY $\leftarrow$ entropy (STATE FEATURE HISTORY $) \times \mathrm{P}($ STATE $)$

FEATURE STATE ENTROPIES $\leftarrow$ FFEATURE STATE ENTROPIES, FEATURE STATE ENTROPY \}

end for

FEATURE CONTEXT ENTROPY $\leftarrow$ entropy(CONTEXT FEATURE HISTORY)

FEATURE MUTUAL INFORMATION $\leftarrow$ FEATURE CONTEXT ENTROPY - sum(FEATURE STATE ENTROPIES)

CUE MUTUAL INFORMATION $\leftarrow$ \{CUE MUTUAL INFORMATION, FEATURE MUTUAL INFORMATION\}

end for

end procedure 
procedure Modulate Cue Weights

CUE WEIGHTS $\leftarrow f$ (CUE MUTUAL INFORMATION, REWARD HISTORY, Attention Distortion Parameter)

end procedure

procedure Estimate Prototype State

SURPRISE LIST $\leftarrow\{\}$

for STATE $\in$ CONTEXT do

STATE MU $\leftarrow$ STATE MU $\times$ CUE WEIGHTS

SURPRISE $\leftarrow-\log (\mathrm{P}(\mathrm{CUE} \mid \mathrm{STATE}$ CUE, STATE COVARIANCE $))$

SURPRISE LIST $\leftarrow$ SSURPRISE LIST, SURPRISE $\}$

end for

if $\min ($ SURPRISE LIST) $>$ State Estimation Surprise Threshold then

STATE $\leftarrow$ Create New State ()

else

STATE PROBABILITIES $\leftarrow \operatorname{softmax}(-\log ($ SURPRISE LIST), State Estimation $\beta)$

STATE $\leftarrow$ CONTEXT[choose(STATE PROBABILITIES)]

end if

end procedure 


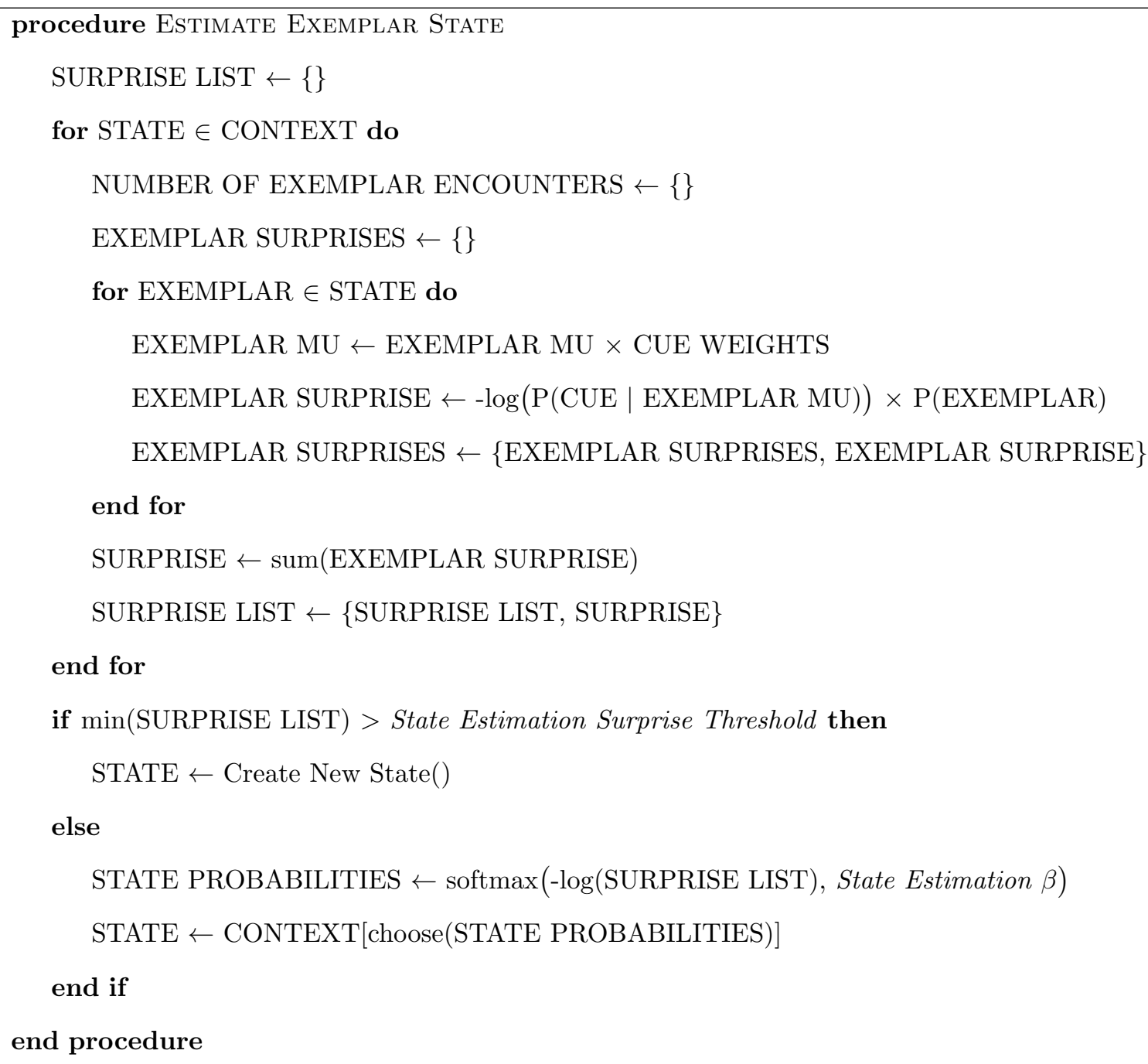




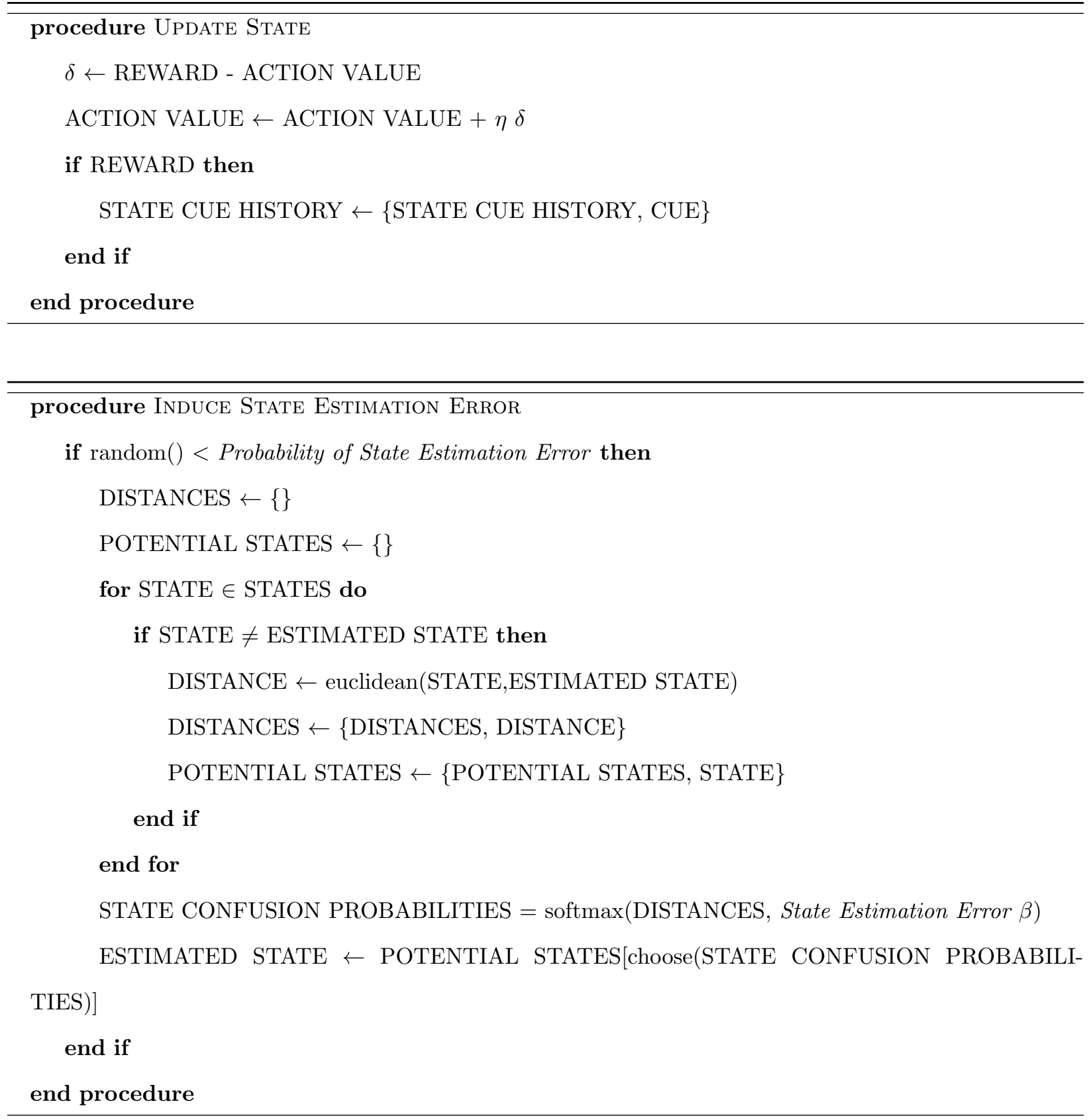




\section{Appendix C: A policy learning artificial neural network produces discriminative errors by failing to recognize context}

While alterations in context recognition by the ProDAtt and ExDAtt models can reproduce behavior of the subpopulation with high discriminative errors, both models do so while still creating states defined by distance. We considered the possibility that the full range of subject behavior can be instead reproduced by a model that simply learns decision boundaries. To simulate a decision boundary model, we built an actor-critic reinforcement learning model, with a three-layer artificial neural network constituting the actor and critic $^{25}$. This ANN is capable of learning, through simple action-reward feedback, nonlinear mappings between inputs and actions. As the dimensionality of the intermediate layer is an order of magnitude higher than the dimensionality of the inputs, it is possible the network can learn to represent states (or classes) as linearly separable in novel contexts ${ }^{56,57}$. Furthermore, task representations can be shaped by manipulating the variance of initialization weights in hidden layers ${ }^{52,58}$. Thus, it is possible that manipulation of hiddenlayer representation in the actor-critic network results in behavioral differences (Fig. S25).

We trained the $\mathrm{ANN}$ on both the $\mathrm{CG}_{1}$ and $\mathrm{CG}_{2}$ tasks. Consistent with prior literature, networks learned faster as the hidden-layer weight initialization variance shifted from a low-variance rich regime to a high-variance lazy regime ${ }^{52}$ (Fig. S26A and B, Fig. S27A and B). However, manipulation of the weight initialization variance had little effect on the initial generalization error, the proportion of discriminative errors remained near $100 \%$, and coefficient value for the discriminative model (D1M in the $\mathrm{CG}_{1}$ ) remained overwhelmingly high (Fig. S26C and D, Fig. S27D). Thus, the ANN recapitulated only behavior of the subpopulation with high discriminative errors.

When examining the activations of hidden layers, we surprisingly found that while the rich regime learned a discriminative representation, the lazy regime formed an exemplar representation. As exemplar representations maintain non-discriminative features, one might expect the lazy regime to produce generalization behavior similar to the ExDAtt model. However, we found that the action layer learned a discriminative representation regardless the variance of initialization weights (Fig. S26F-G, Fig. S27E-G). This meant that

action selection was dictated by discriminative decision boundaries, and thus produces discriminative errors regardless the hidden layer representation. In effect, the lazy regime ANN is qualitatively equivalent to an ExDAtt model lacking context recognition. 


\section{Supplemental Figures}
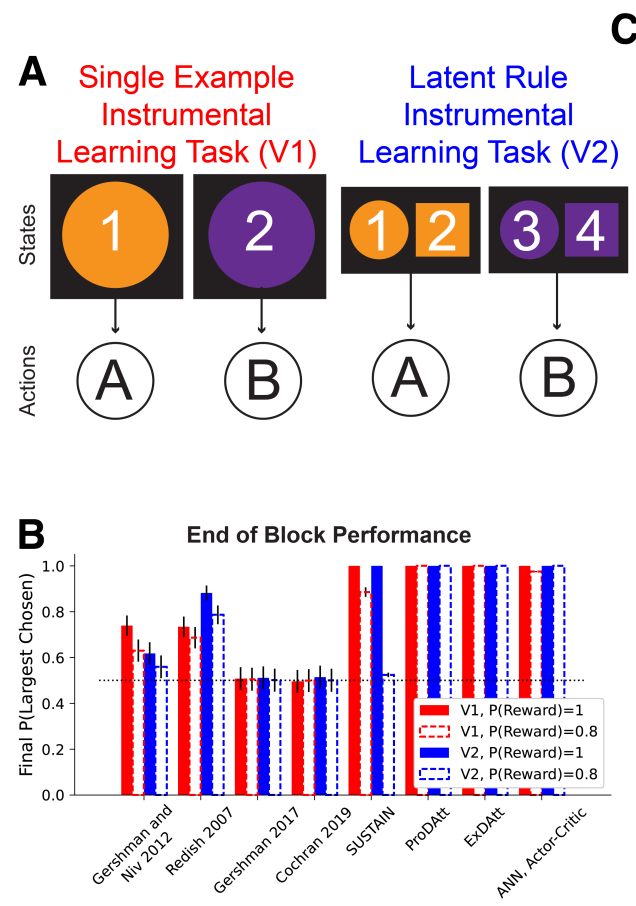
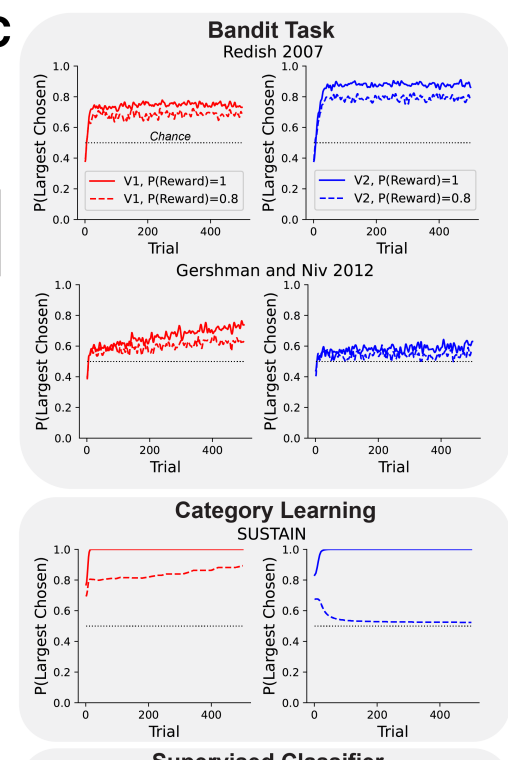

Supervised Classifier

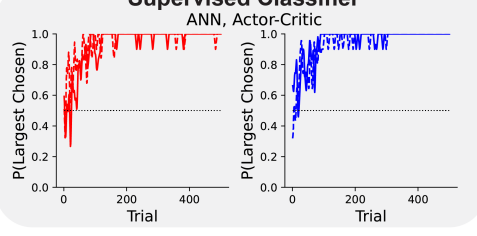

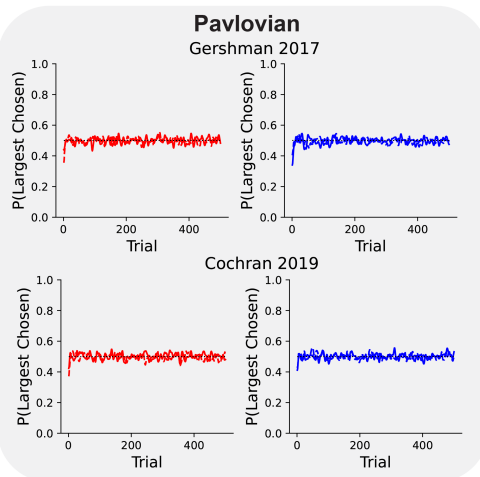

Instrumental Latent Rules

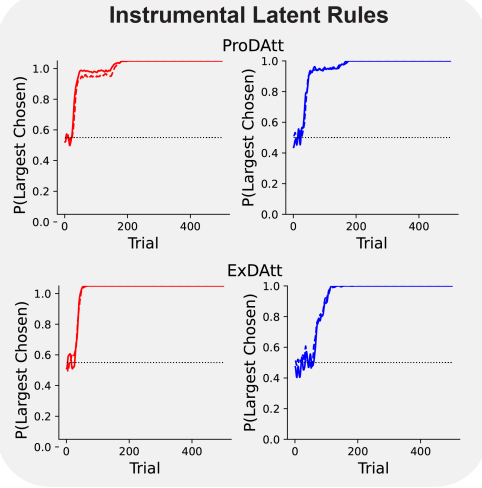

Figure S1: Previous models achieve varying success when learning latent rules with stochastic rewards. Supervised and Unsupervised STratified Adaptive Incremental Network, "SUSTAIN," the "prototype states with discriminative attention" (ProDAtt) model, the "exemplar states with discriminative attention" (ExDAtt) model, artificial neural network (ANN). (A) We implemented two simple multi-state instrumental learning tasks. In the first task, there were two examples and two actions, with each state deterministically rewarded for a unique action. The second task had the same action-reward contingencies, but involved two examples for each state, with color as the latent rule determining state-membership. Each task had a version where rewards were deterministic, and another where rewards were stochastic with an $80 \%$ probability. While a basic Rescorla-Wagner reinforcement learning algorithm could trivially learn these action-reward contingencies, it relies on a pre-specified states. The models tested on these tasks had to learn states to organize action selection, in addition to learning the action-reward contingencies. (B) Performance at the end of 500 trials. Previous models designed to learn the latent structure of bandit rewards (Redish 2007, Gershman and Niv 2012) performed just above chance. Those designed to learn the latent states underlying Pavlovian conditioning (Gershman 2017, Cochran 2019) performed at chance. The SUSTAIN model of category learning quickly learned the deterministic version of each task, but struggled with the stochastic single example task, and fell to chance with the stochastic latent rule task. The artificial neural network (ANN) implementation of actor-critic RL, the ProDAtt and the ExDAtt performed perfectly on all version of the tasks. This demonstrates previous latent-state and category learning models are insufficient for instrumental learning tasks which the ANN, the ProDAtt and the ExDAtt models quickly master. (C) Individual learning curves for each model demonstrates the learning over trials. 
Initial Subject Sample ( $n=109)$, Amazon Mechanical Turk
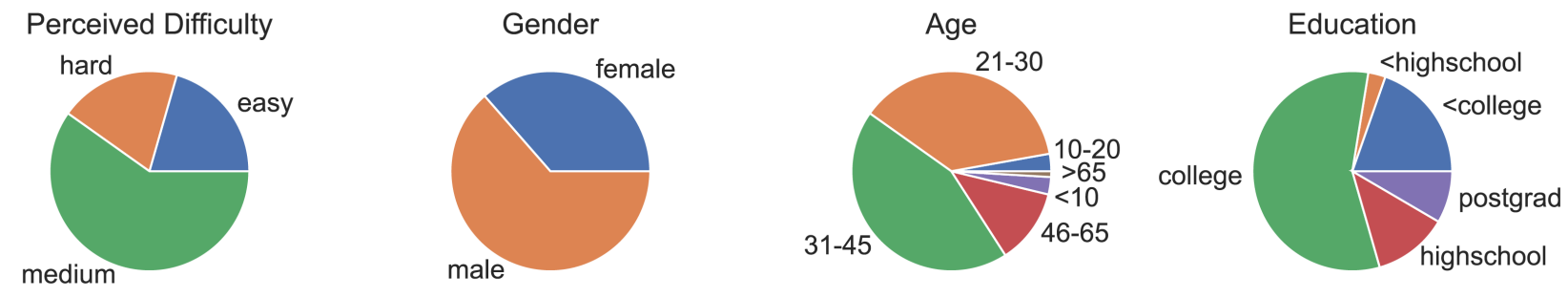

\section{Replication Subject Sample ( $\mathrm{n}=156)$, Prolific}
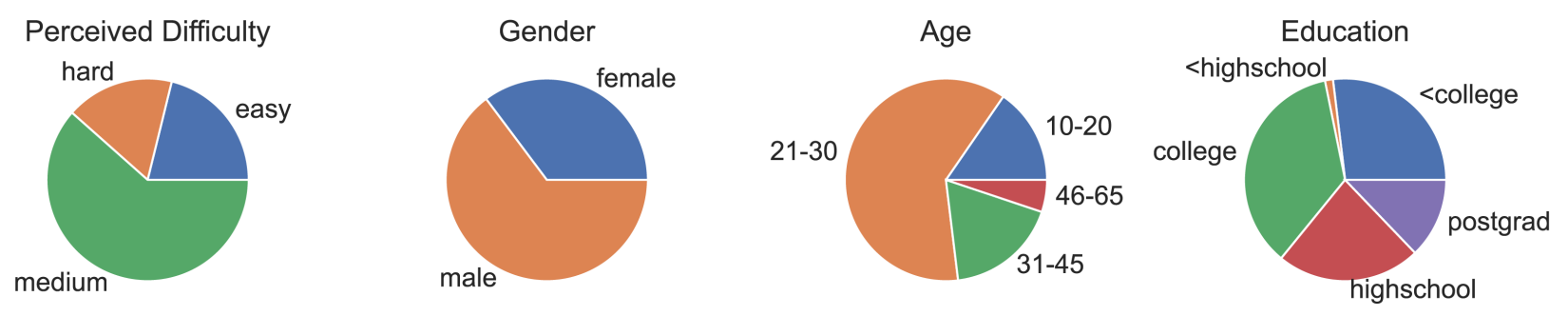

Figure S2: Subject demographics for Experiment 1. Demographics were similar across platforms for the initial population on mTurk (top) and replication population on Prolific (bottom). 

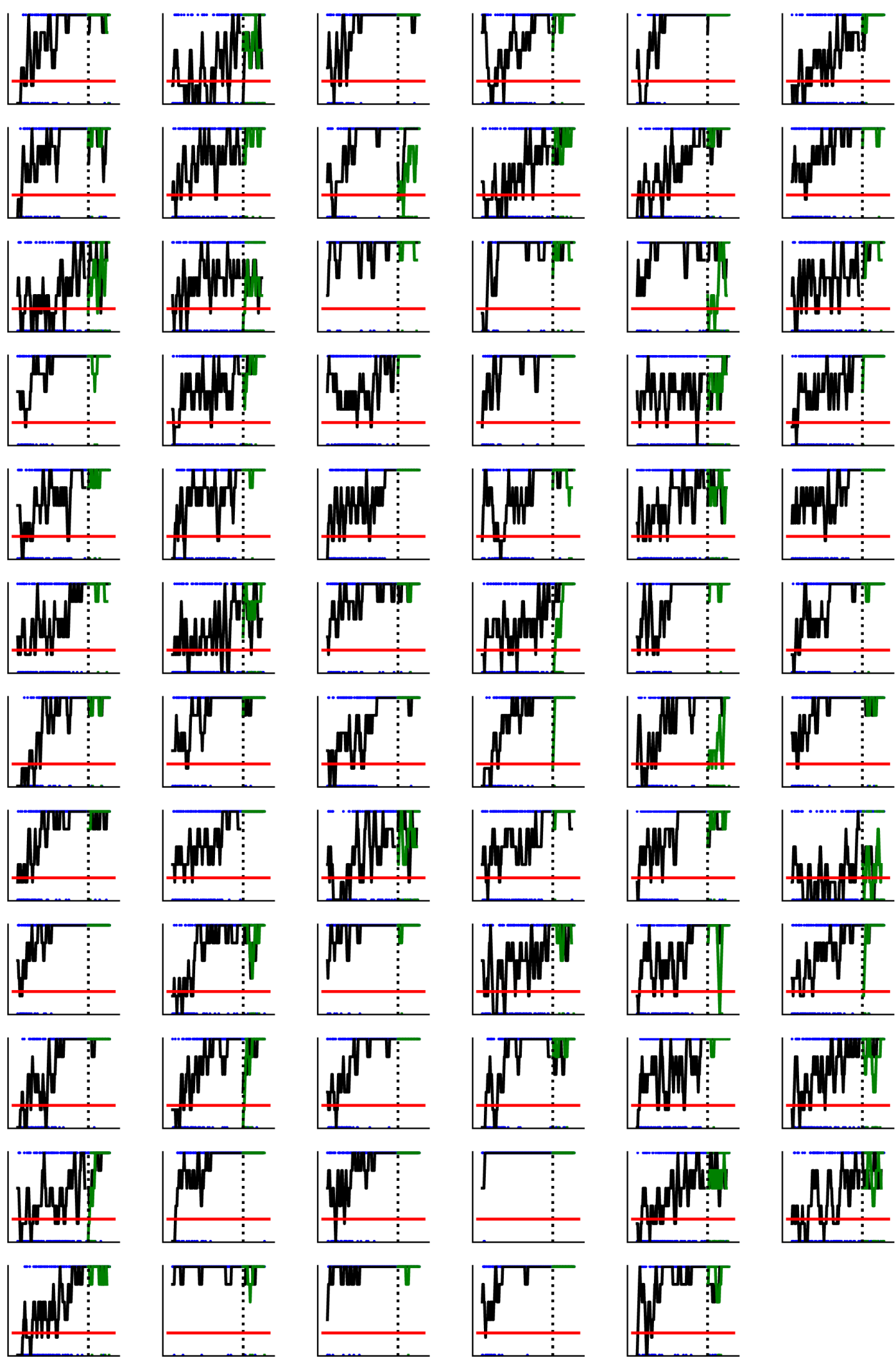

Figure S3: Individual learning curves for the initial sample of Experiment 1. Trial-by-trial correct/incorrect (blue points), smoothed performance on initial examples (black), smoothed performance on novel examples (green) block transition (vertical dotted), chance (red horizontal). 
|

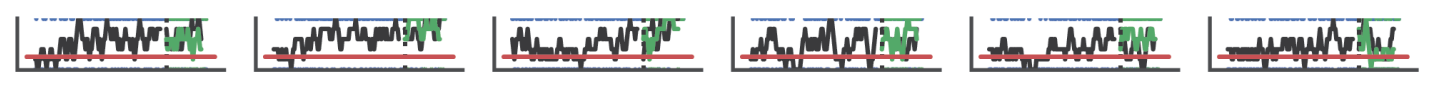

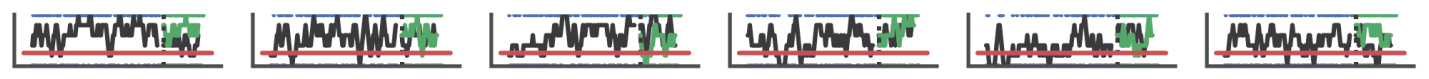

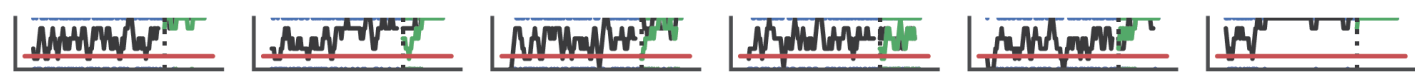
15 |

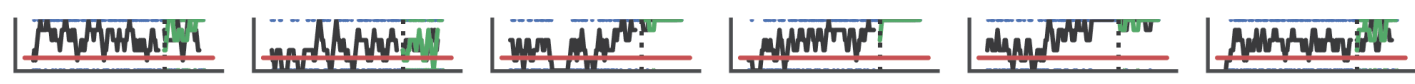

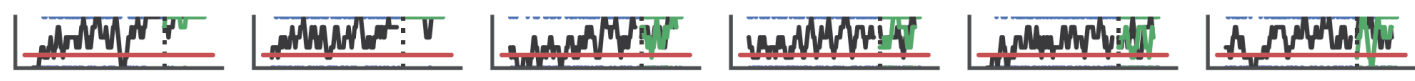

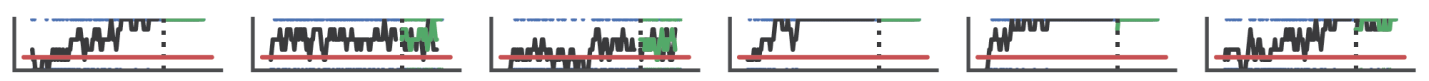
1) NDV:-

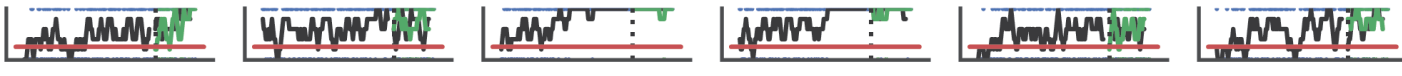

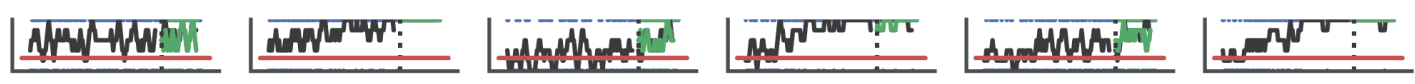

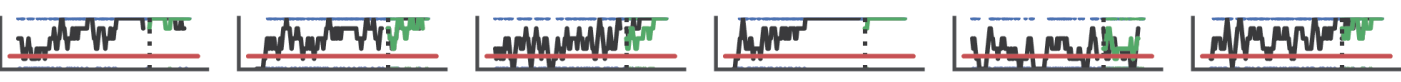
Li: :

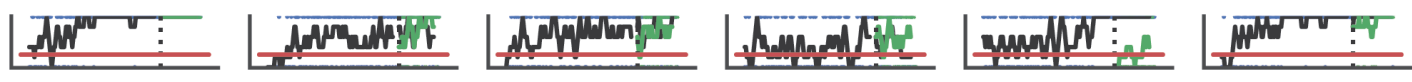

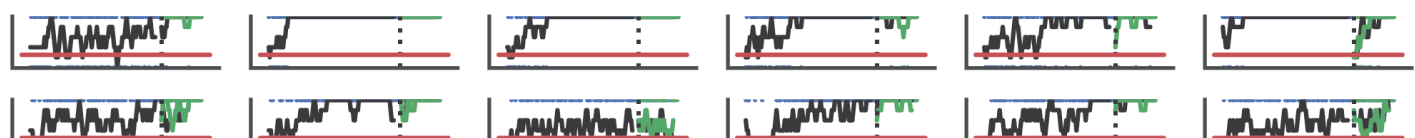

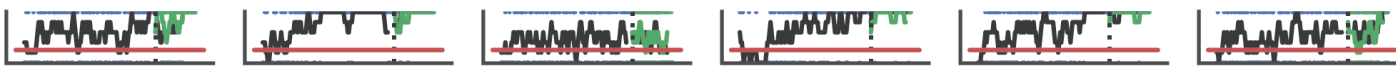

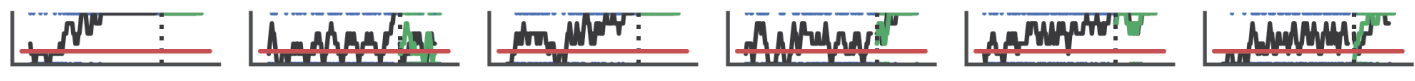
|

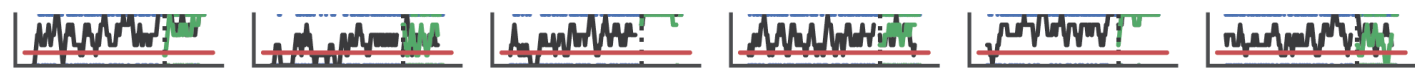
| L

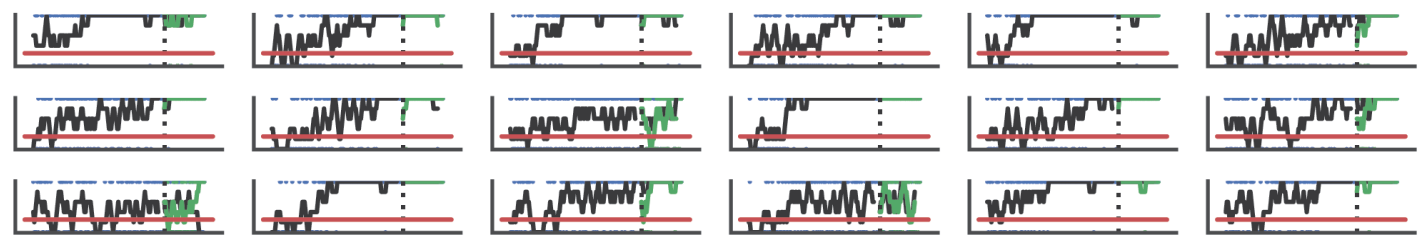

Figure S4: Individual learning curves for the replication sample of Experiment 1. 


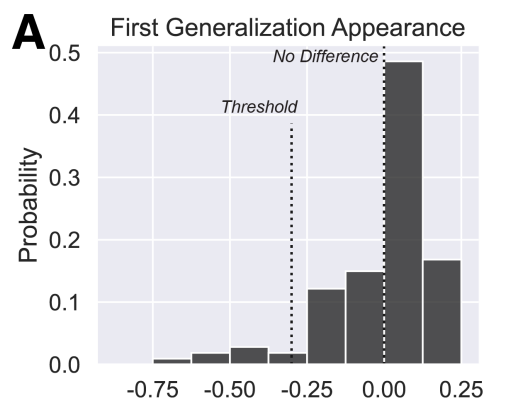

$\mathrm{P}$ (Correct | Novel, First Gen Appearance) $\mathrm{P}$ (Correct | Learned, First Gen Appearance)

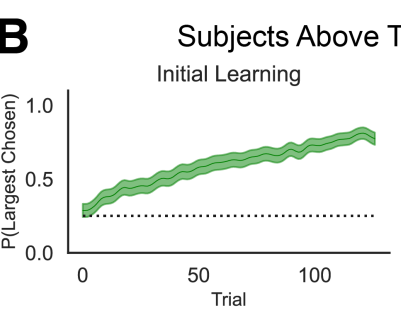

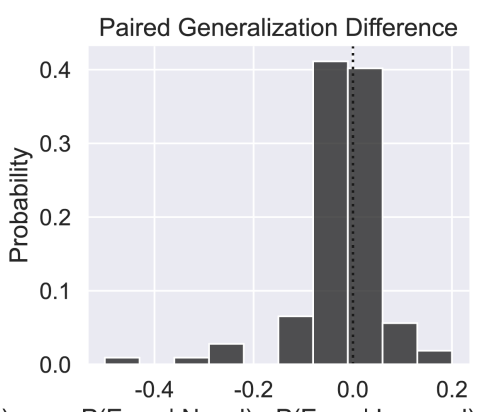

$\mathrm{P}$ (Error | Novel) - P(Error | Learned)

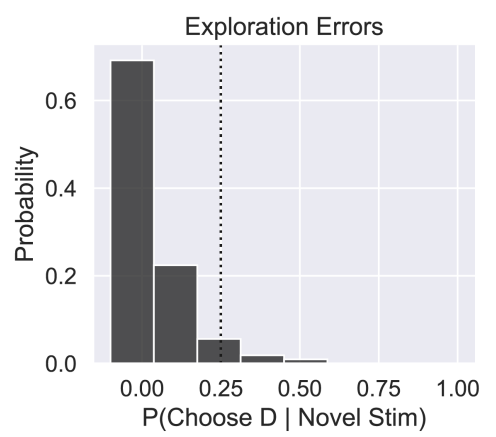

Subjects BelowThreshold $(\mathrm{N}=8)$

Novel Example Generalization
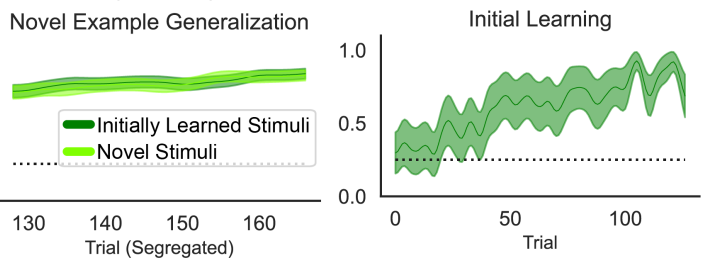

Novel Example Generalization

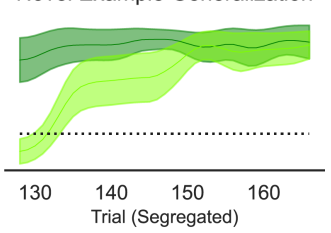

Figure S5: Summary statistics and subpopulations for the initial sample of Experiment 1. (A) Histograms of generalization metrics. (B) Learning curves for subjects above (left) and below (right) the first generalization appearance threshold.
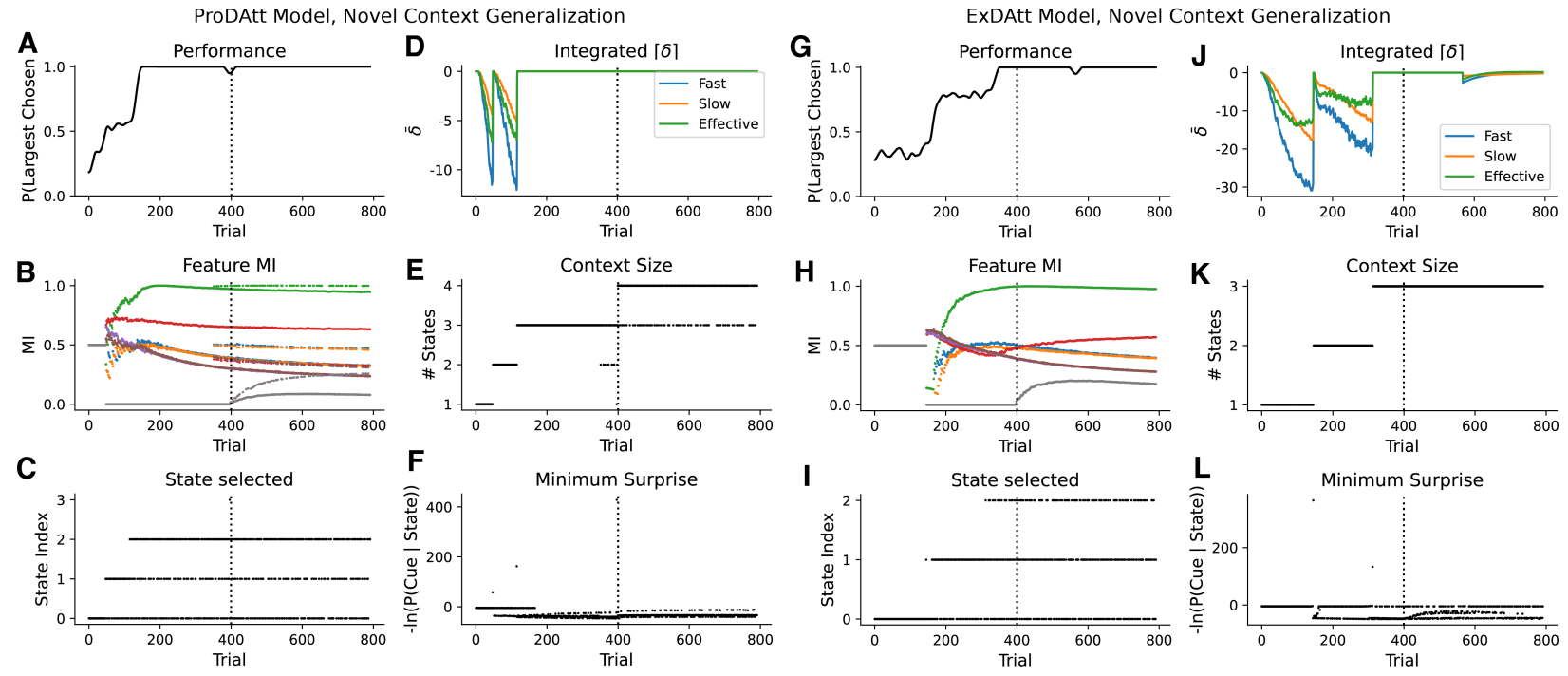

Figure S6: Single agents learn novel example generalization in Experiment 1. Points are individual trials. (A) A ProDAtt model agent learns the initial representations then transitions to generalization stimuli. (B) The feature mutual information stabilizes as states are created, and adjusts as new examples appear. (C) Four states are learned, of which three are repeatedly used through the transition. (D) The integrated RPE spikes repeatedly until the agent learns a stable set of state representations. (E) The context size fluctuates between trials. (F) The minimum surprise level spikes prior to the creation of new states. (G-L) Same for the ExDAtt model. 

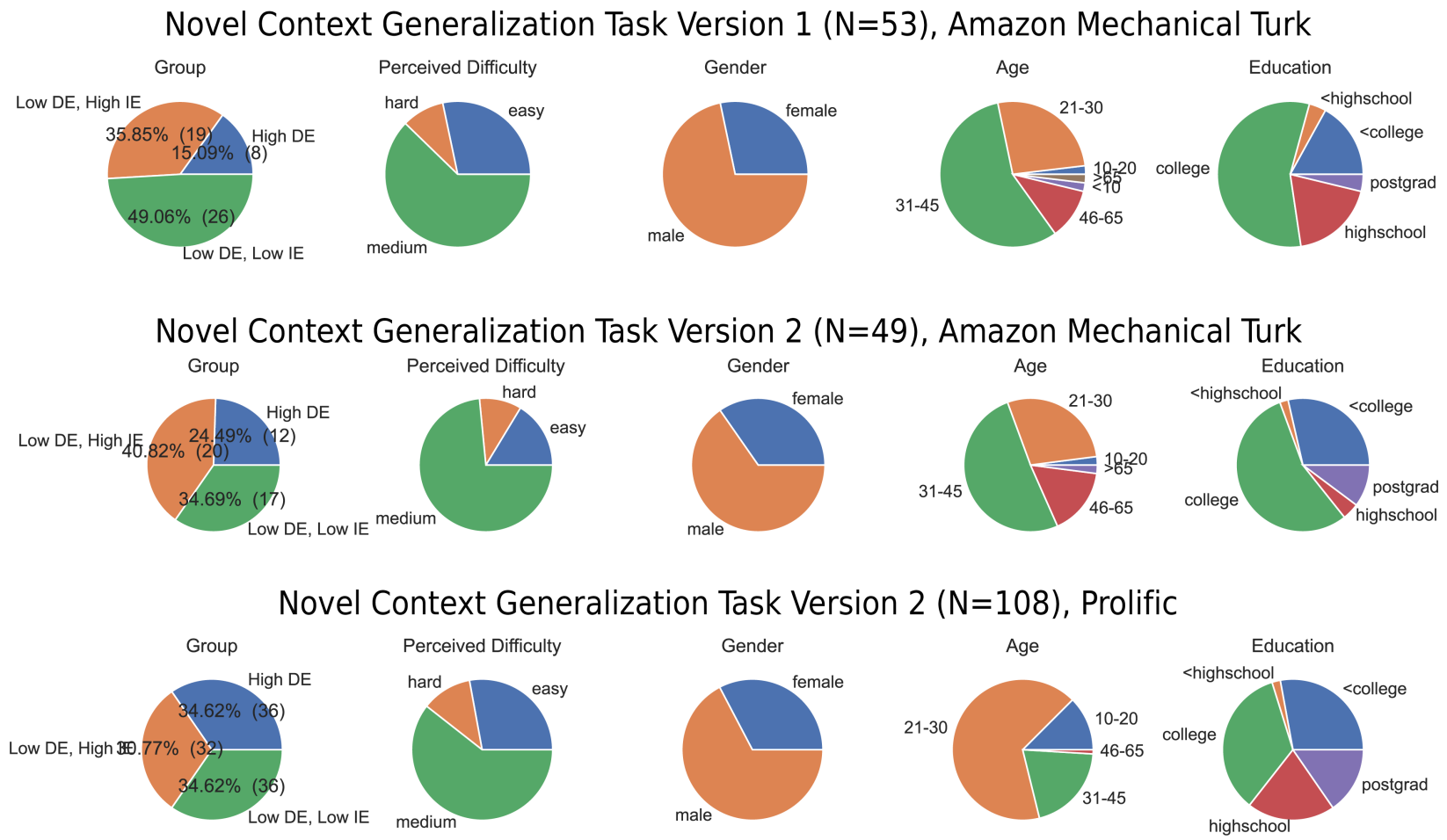

Figure S7: Subject demographics for Experiments 2 and 3. Demographics for the novel context generalization task were similar across platforms for version 1 on mTurk (top), version 2 on mTurk (middle) and the replication of version 2 on Prolific (bottom). 

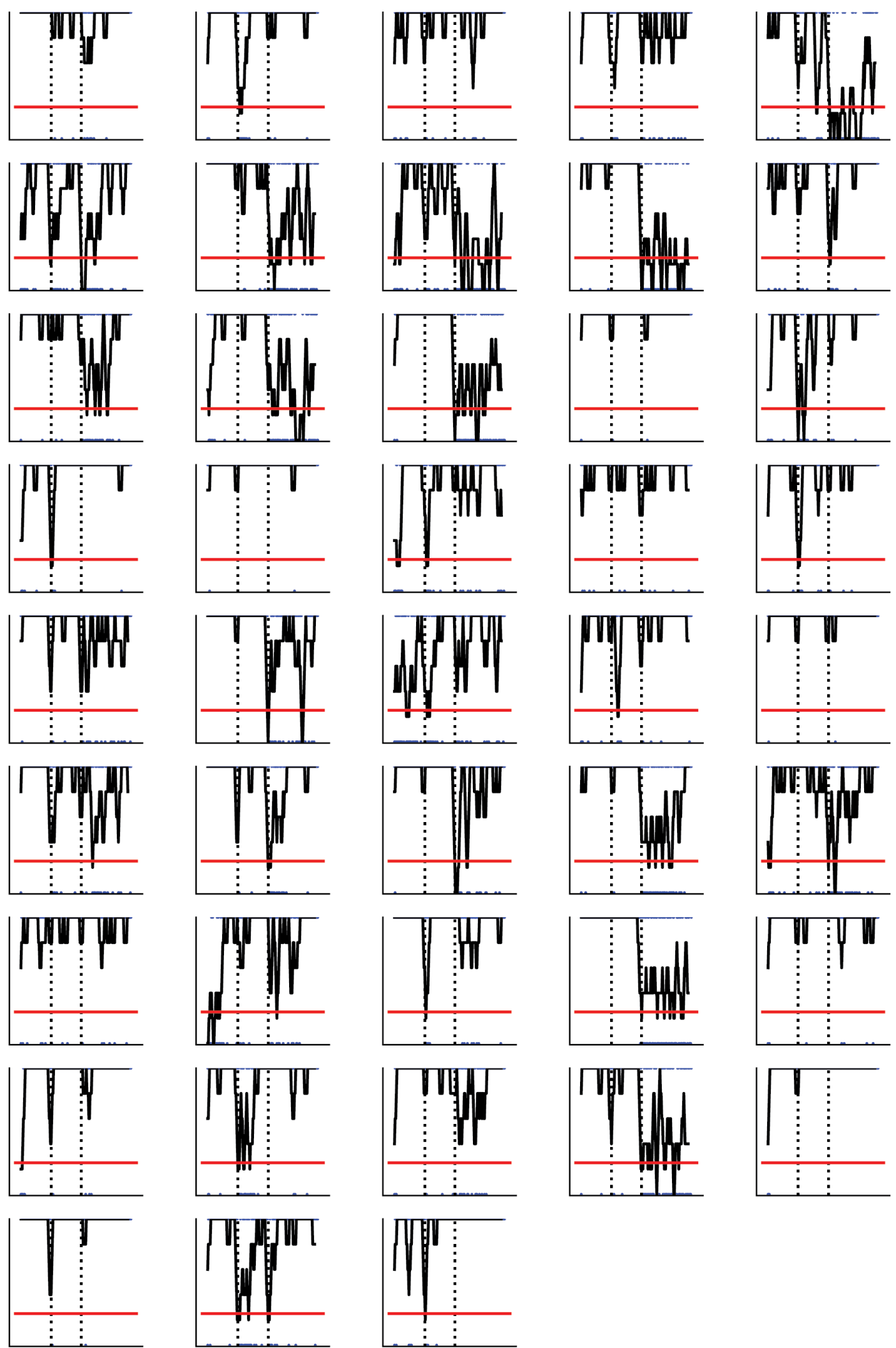

Figure S8: Individual learning curves for the novel context generalization task version 1 subjects. 
A

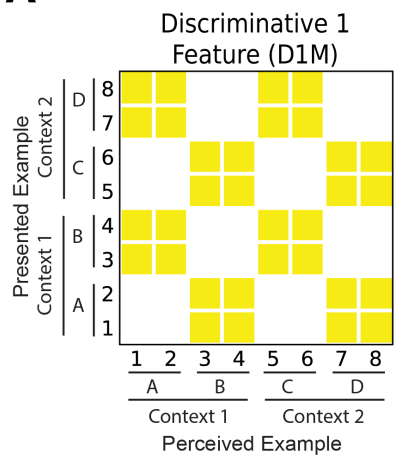

Idealized Attention Model Feature Distances

Discriminative 2

Features (D2M)

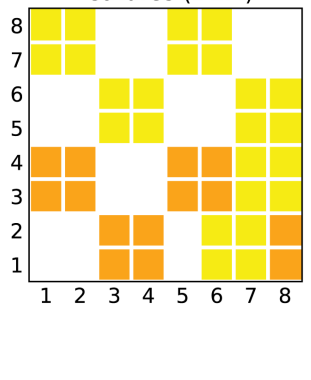

(P)rototype

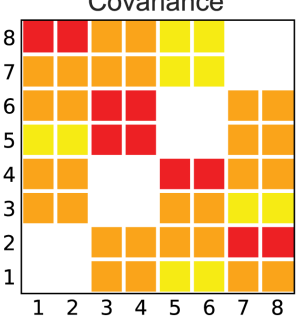

(A)II Features

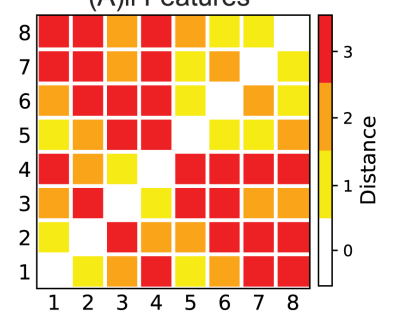

B

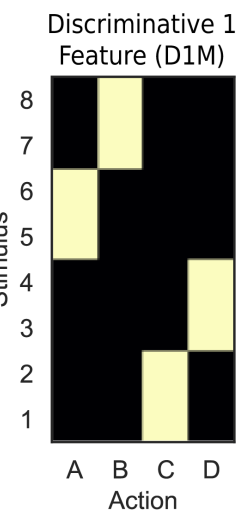

Idealized Attention Model Confusion Matrices

Discriminative 2

Features (D2M)
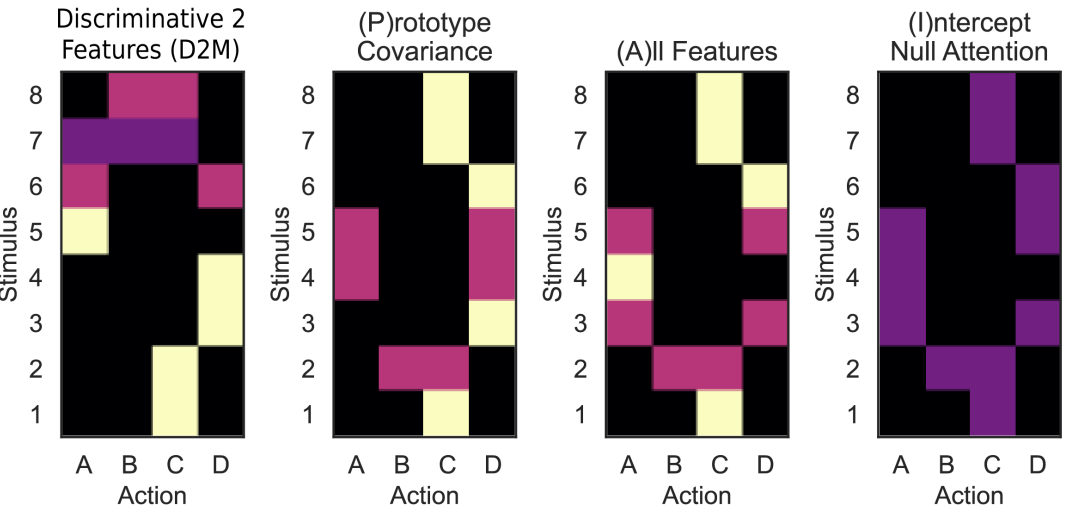

C
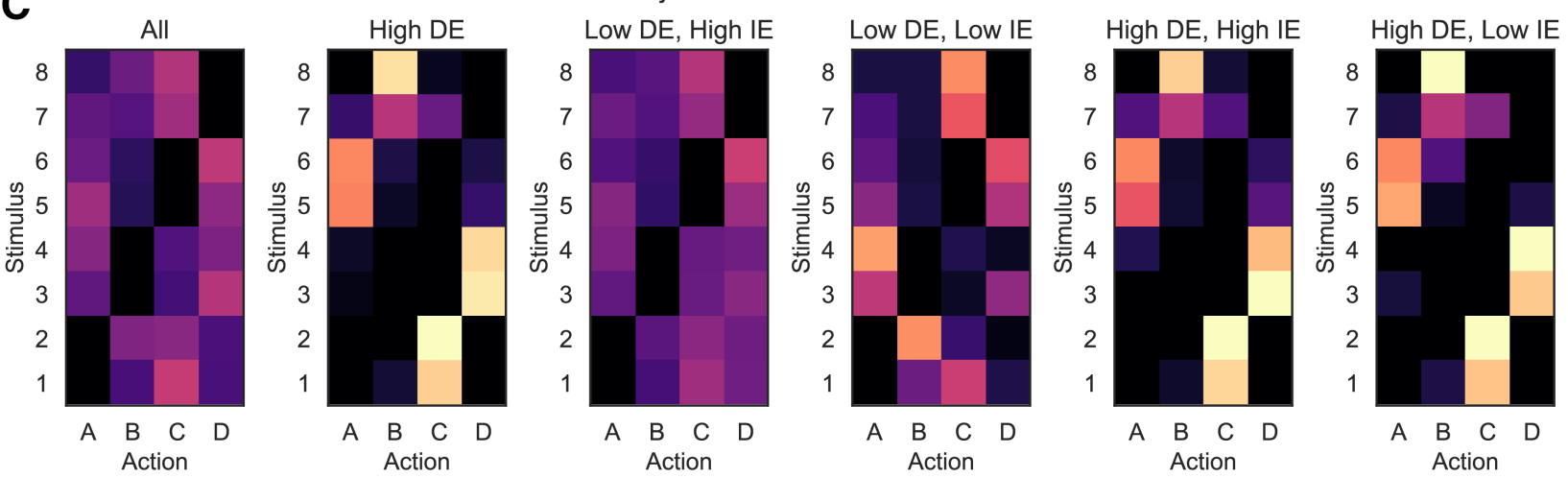

Figure S9: Novel context generalization task version 1 confusion matrices. (A) Idealized attention model feature distances. (B) Idealized attention model matrices used to fit behavior. (C) The observed confusion matrices for each group. 


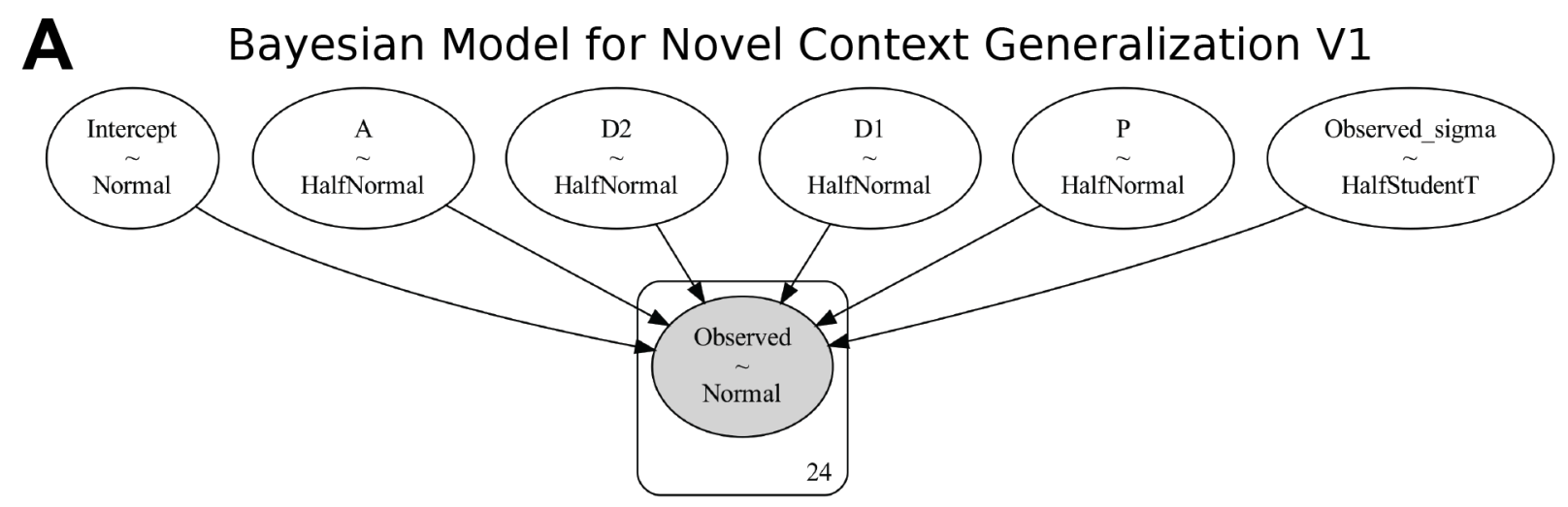

B

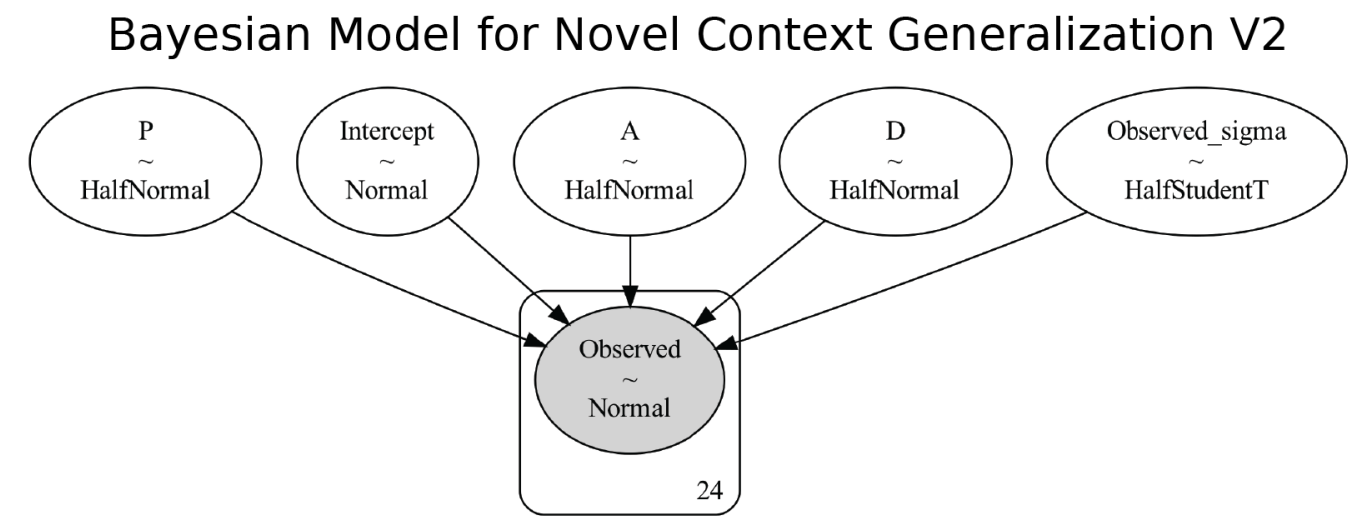

C Alternative Bayesian Model for Novel Context Generalization V2

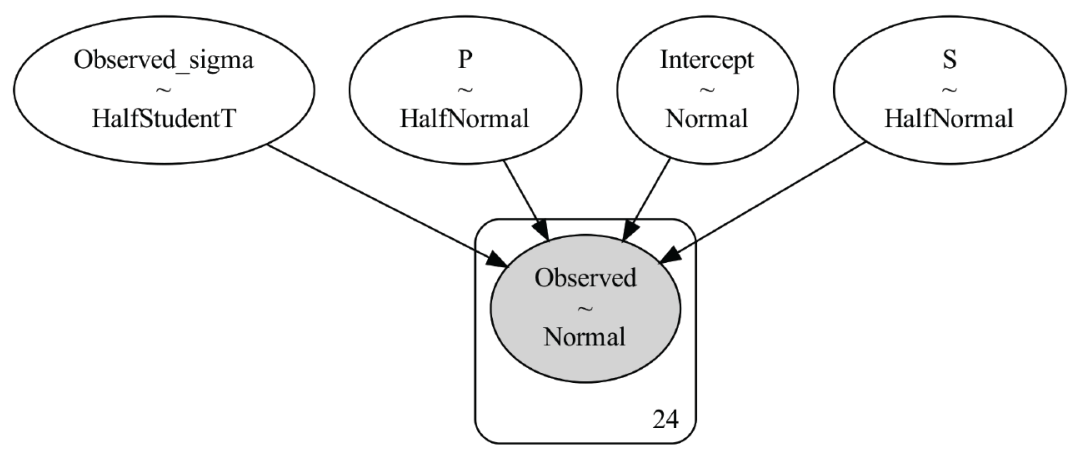

Figure S10: Structure of Bayesian models used to fit the idealized confusion matrices to behavioral data. All-feature attention, "A," second discriminative model (D2M), first discriminative model (D1M), prototype covariance, "P," state bias "S." (A) Bayesian model for novel context generalization task version 1, used in Experiment 2. (B) The main Bayesian model for the novel context generalization task version 2, used in Experiment 3. (C) The alternative Bayesian model for the novel context generalization task version 2 . 

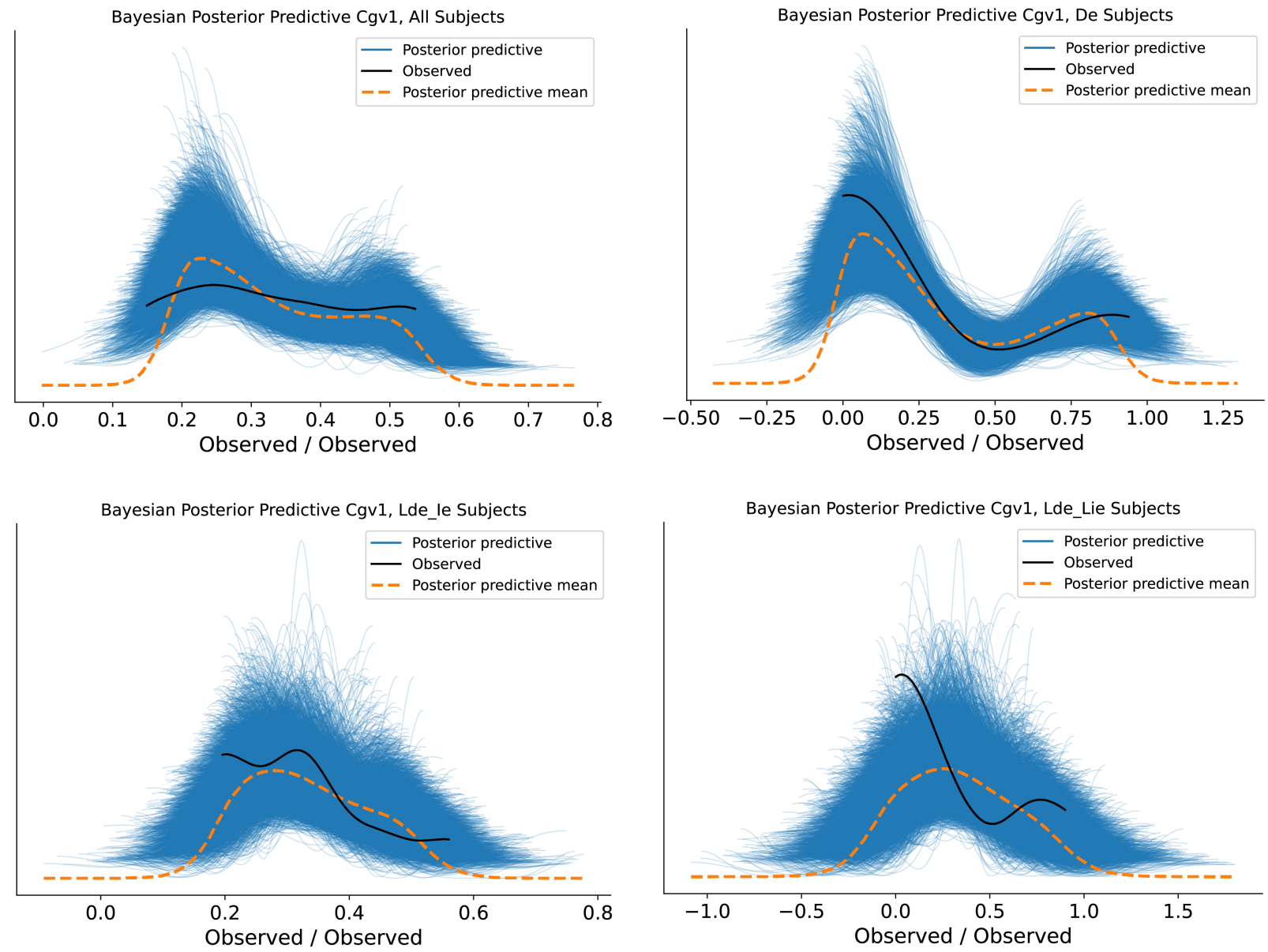

Figure S11: Novel context generalization version 1 posterior predictive distributions by population. 

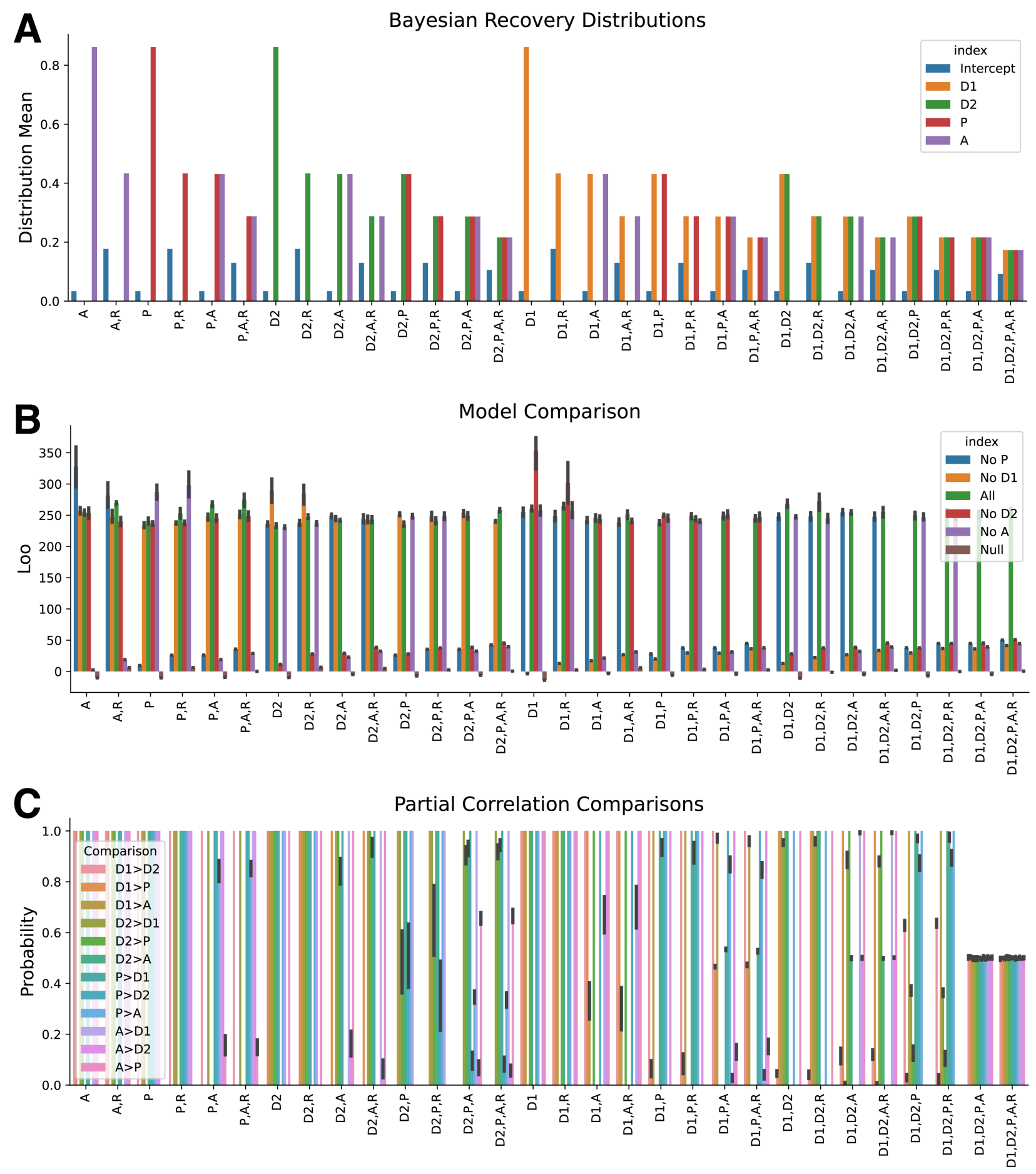

Figure S12: Novel context generalization task version 1, idealized attention model recovery using Bayesian linear regression. The x-axis indicates the combination of idealized attention models used to generate the data. (A) The coefficient distribution means across 10 draws with noise indicate recovery of the underlying data source. Though there are error bars, variation across fits is so small that they are not visible. (B) Use of model comparison also recovers the data sources, with those models lacking the underlying sources performing worse than those with the sources. Error bars indicate the standard deviation. (C) Pairwise comparison of the partial correlation distributions indicate a higher probability for the underlying sources. Error bars indicate the standard deviation. 


\section{Model Comparison}

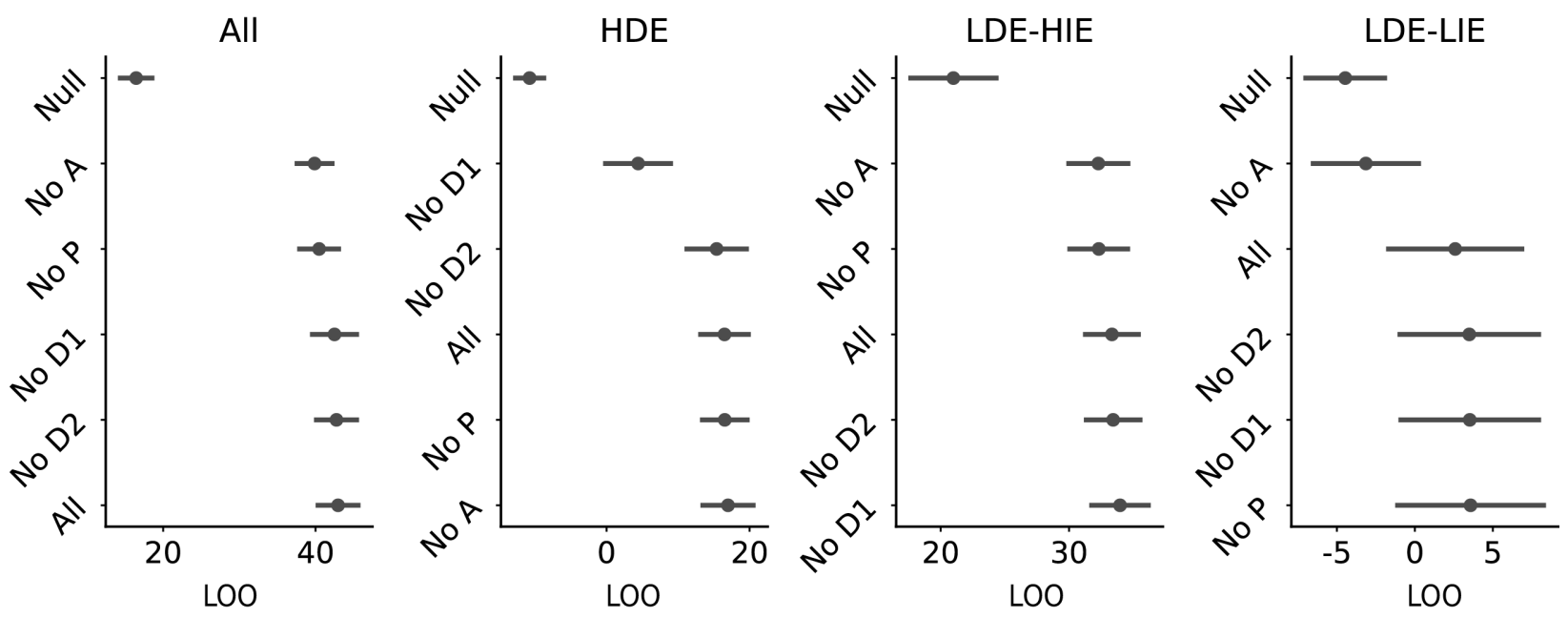

Figure S13: Novel context generalization task version 1 model comparison We quantified the performance of each model using Pareto smoothed importance sampling leave-one-out cross-validation. The further left, worse the fit without that idealized attention model. Null indicates a model with only an intercept distribution. The model comparison is shown for each subpopulation. 


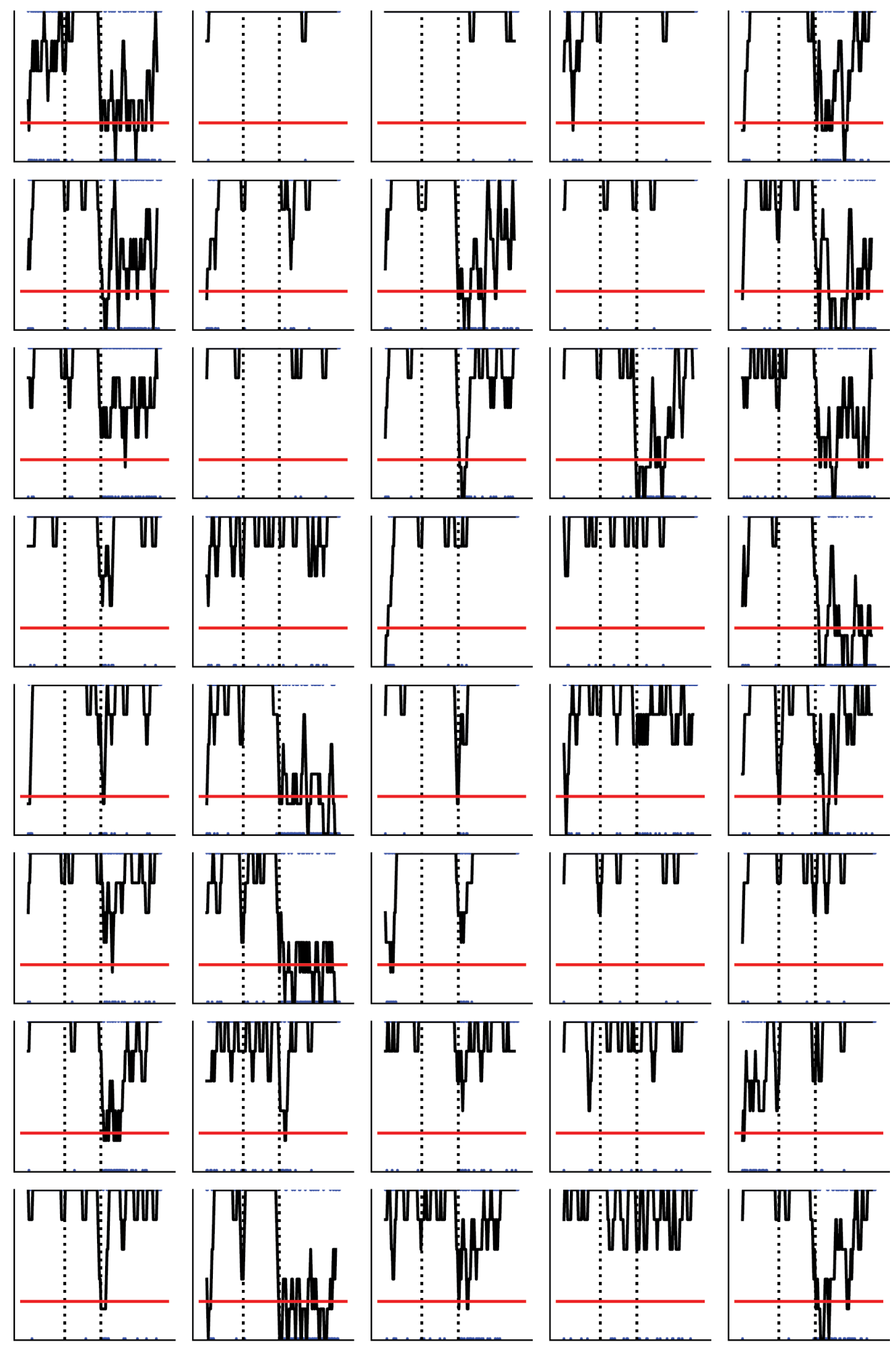

Figure S14: Individual learning curves for the novel context generalization task version 2 initial subject sample. 


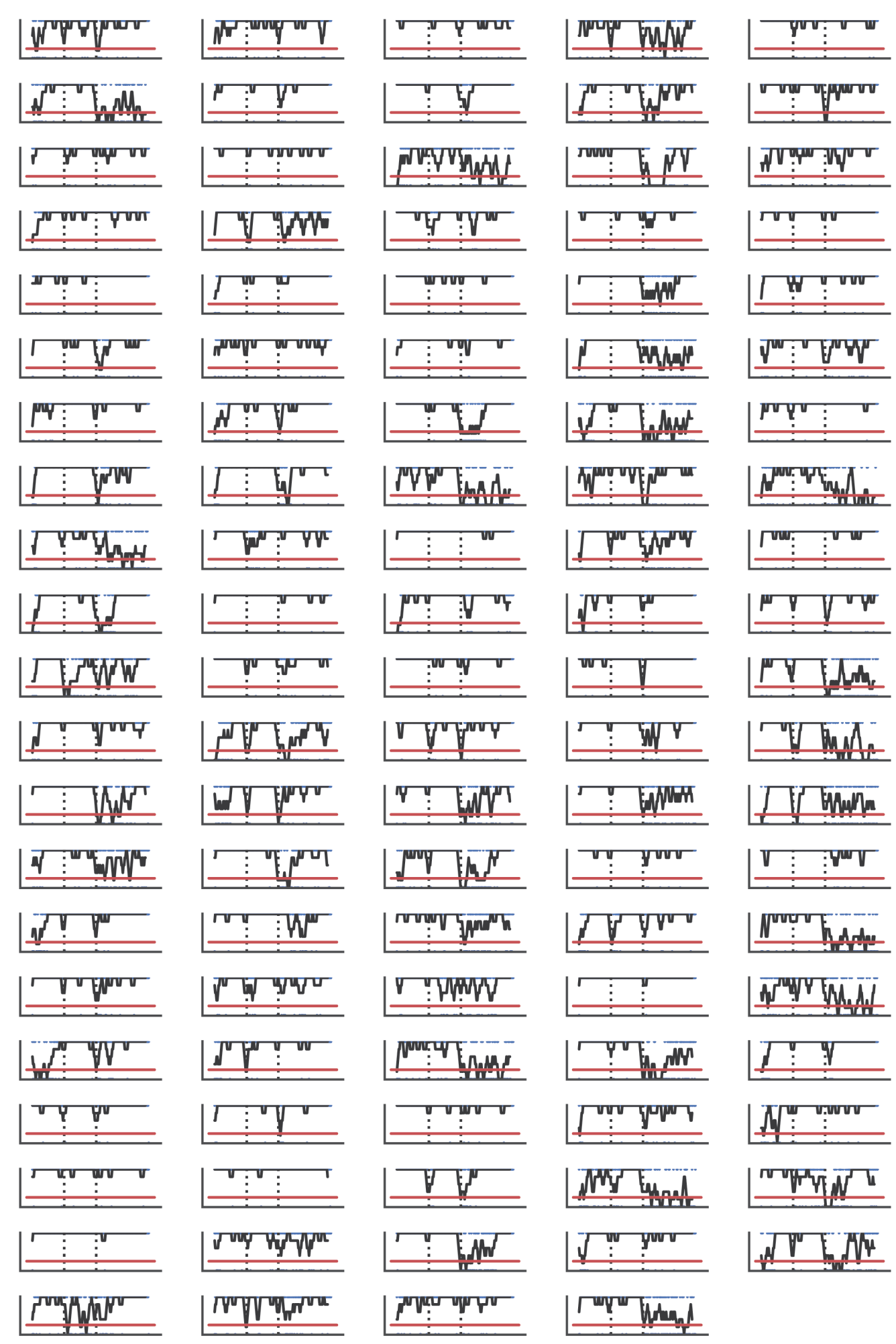

Figure S15: Individual learning curves for the novel context generalization task version 2 replication subject sample. 


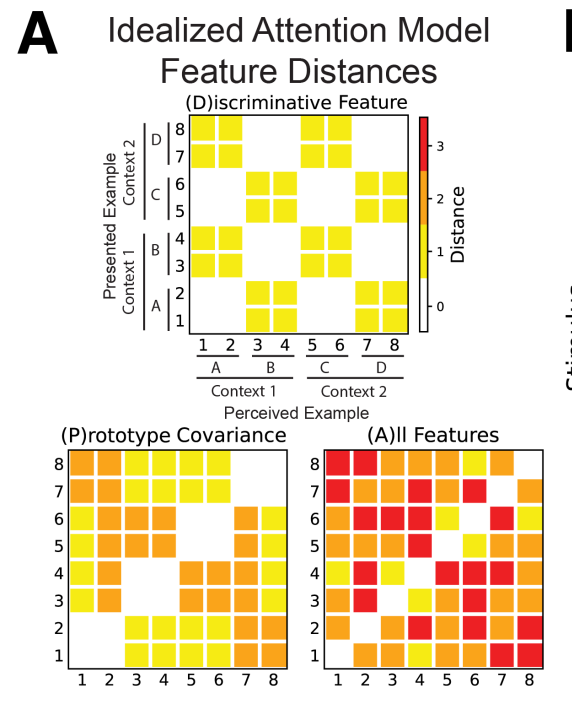

B Idealized Attention Model Confusion Matrices
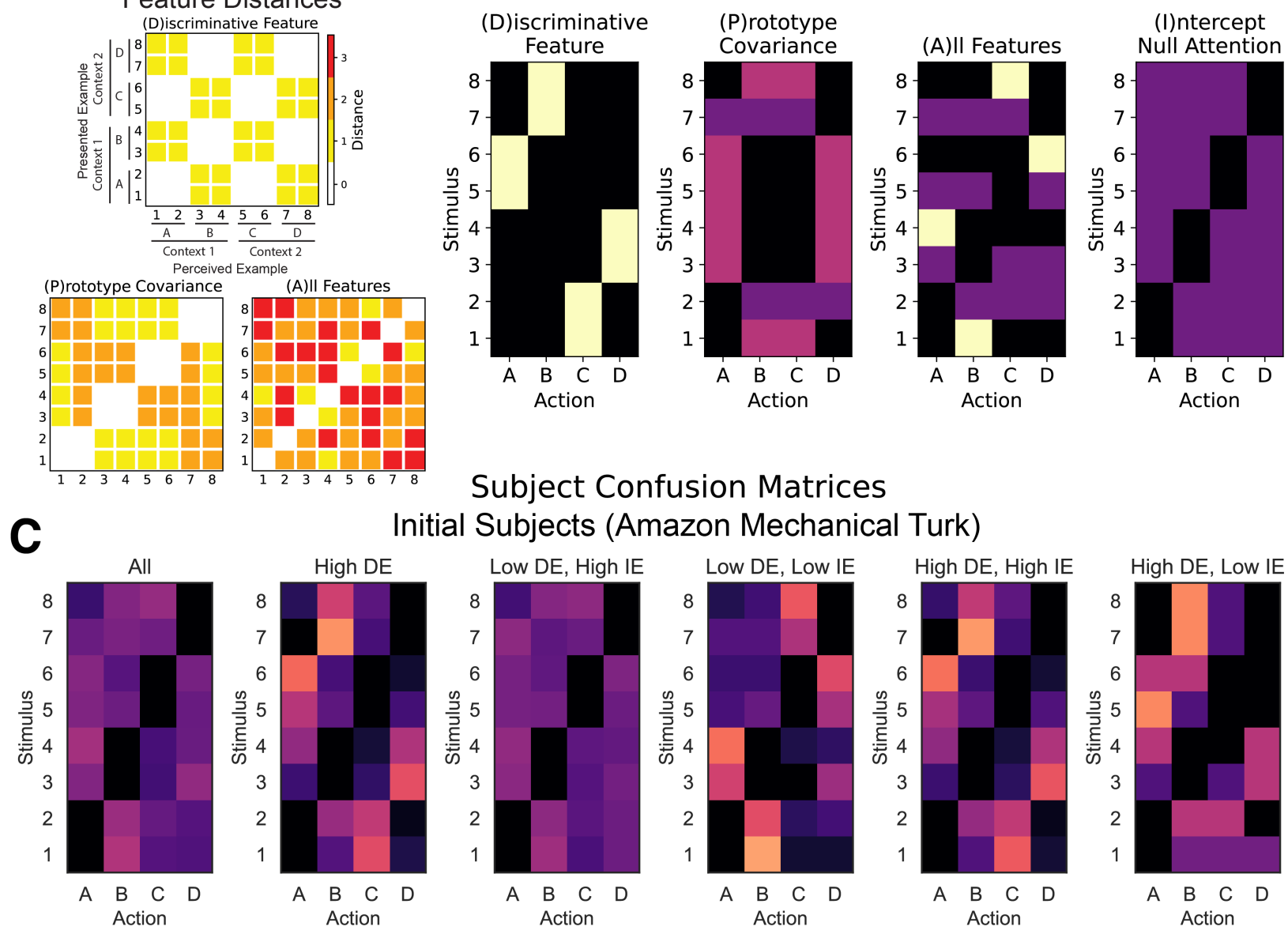

Initial Subjects (Amazon Mechanical Turk)
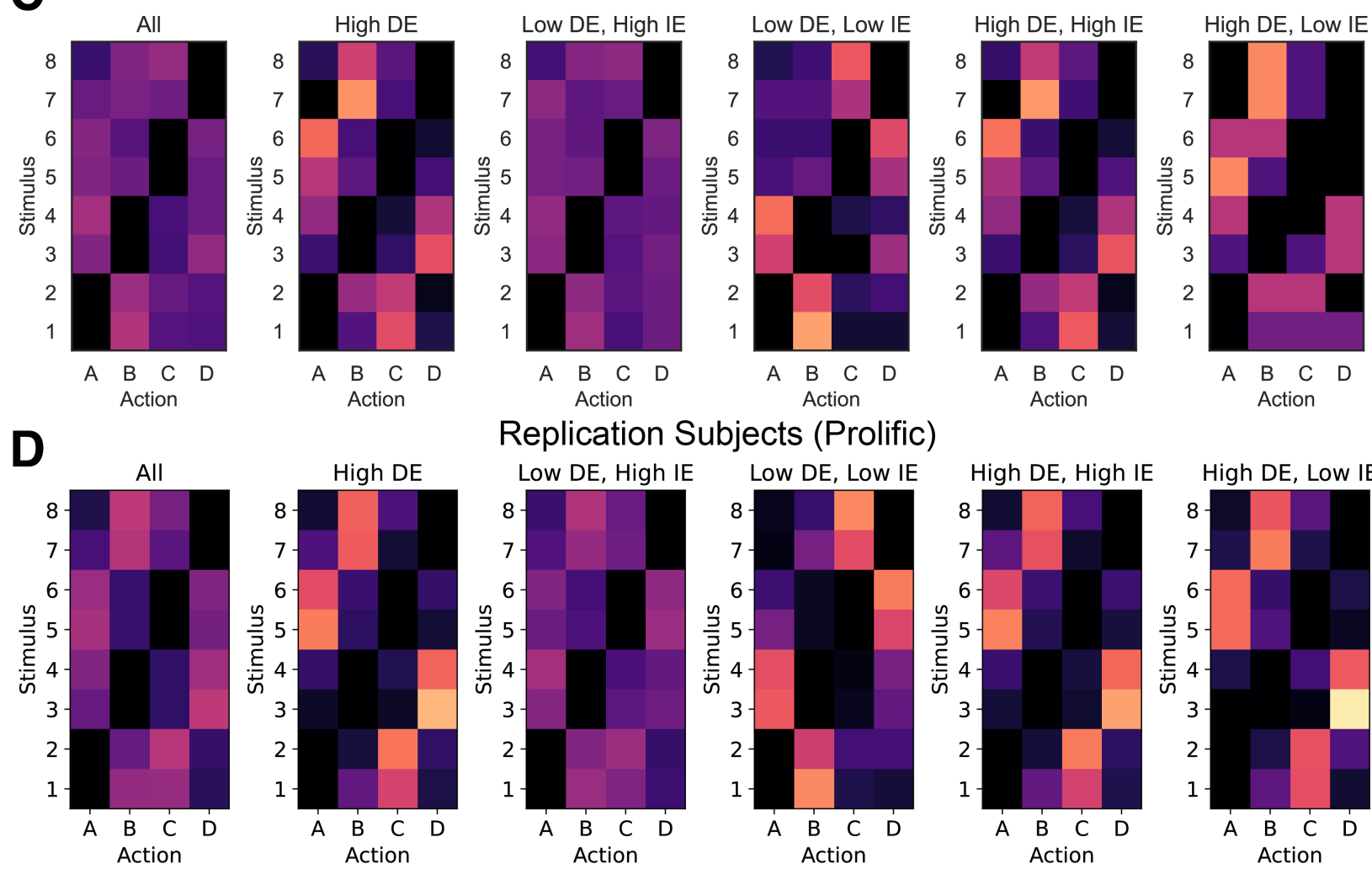

\section{Replication Subjects (Prolific)}
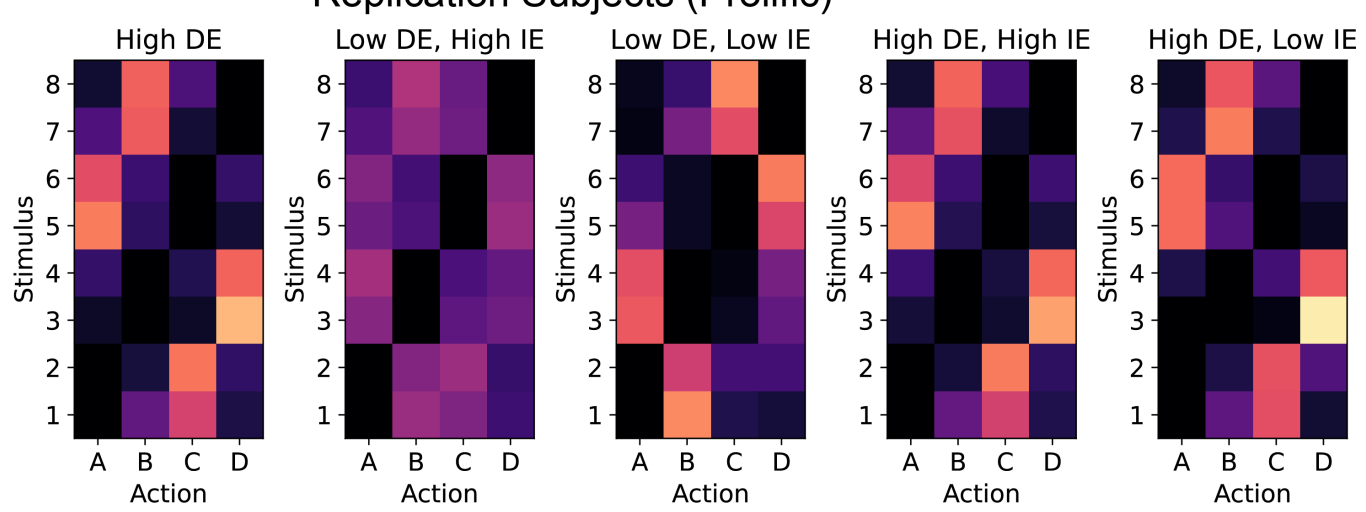

Figure S16: Novel context generalization task version 2 confusion matrices. (A) Idealized attention model feature distances. (B) Idealized attention model matrices used to fit behavior. (C) Initial sample population confusion matrices by group. (D) Replication sample population confusion matrices by group. 

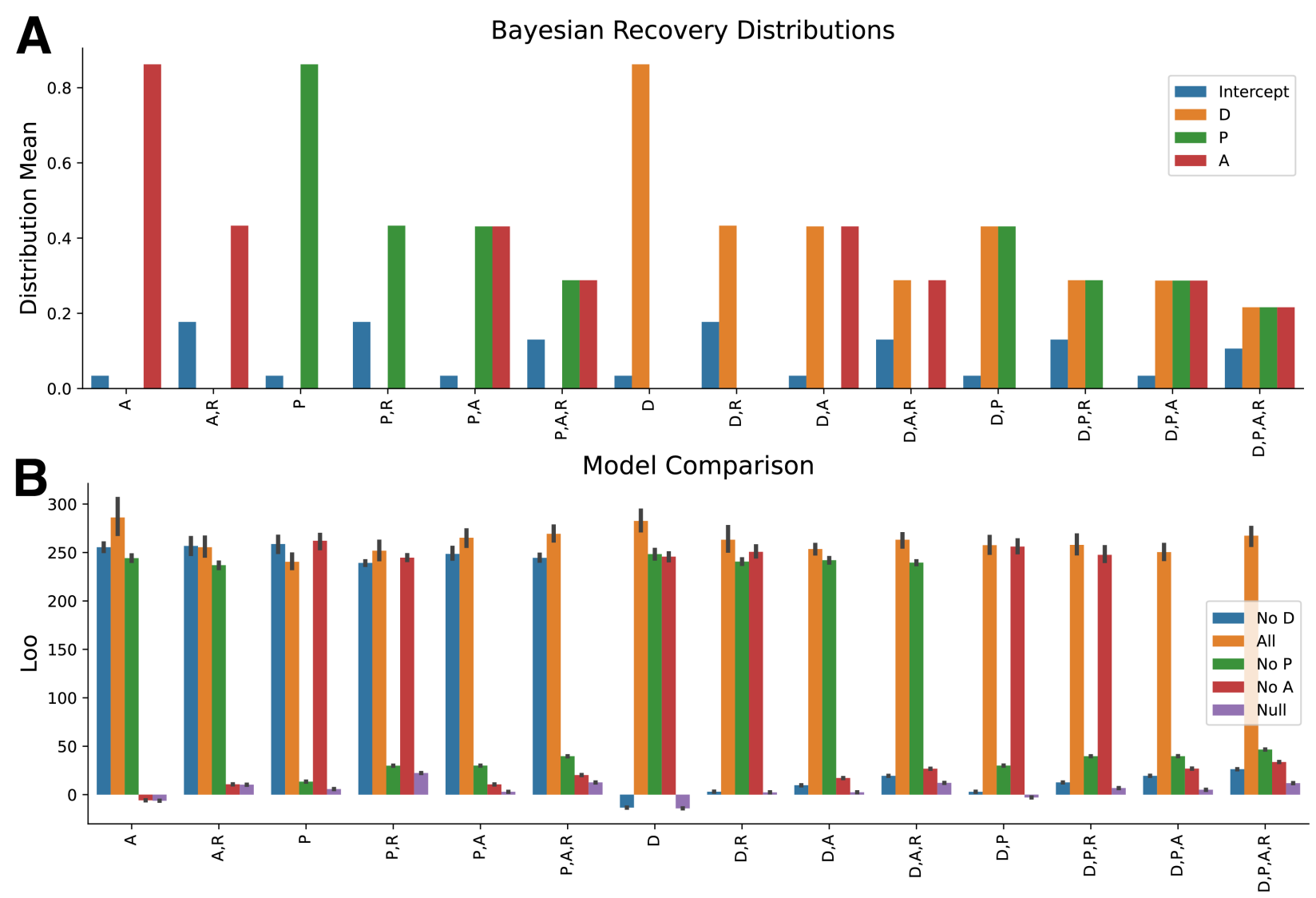

Figure S17: Novel context generalization task version 2, idealized attention model recovery using Bayesian linear regression. The x-axis indicates the combination of idealized attention models used to generate the data. (A) The distribution means across 10 draws with noise indicate recovery of the underlying data source. Error bars are too small to be visible. (B) Use of model comparison also recovers the data sources, with those models lacking the underlying sources performing worse than those with the sources. Error bars indicate the standard deviation. 

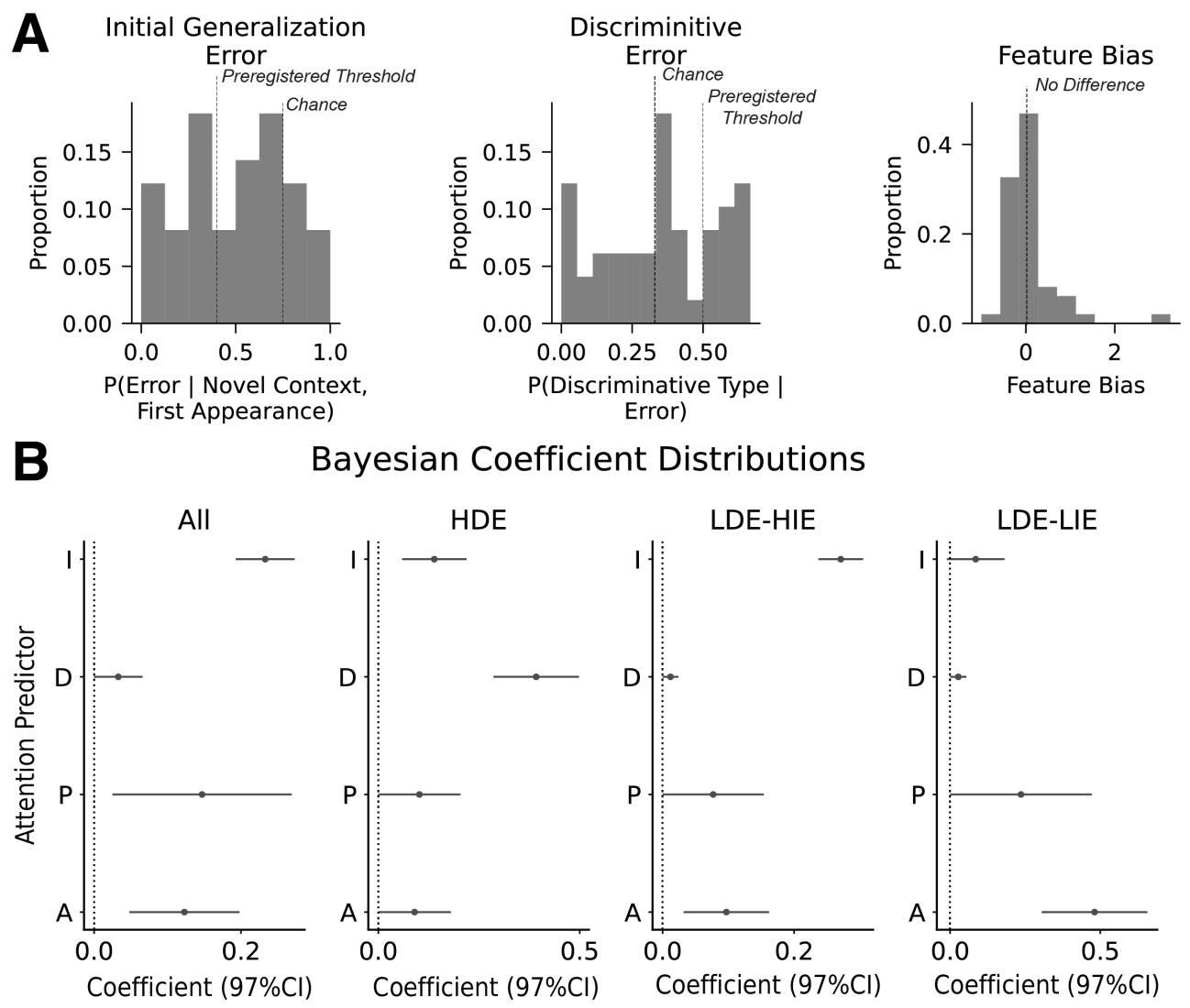

C

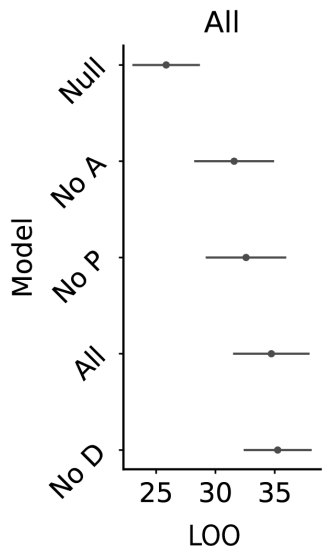

\section{Model Comparison}

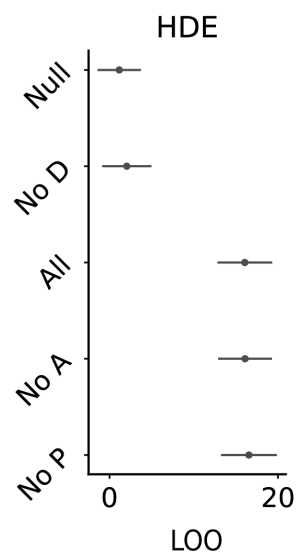

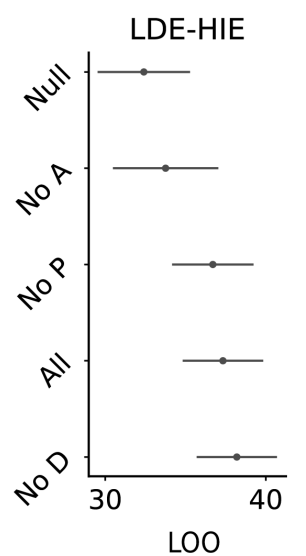

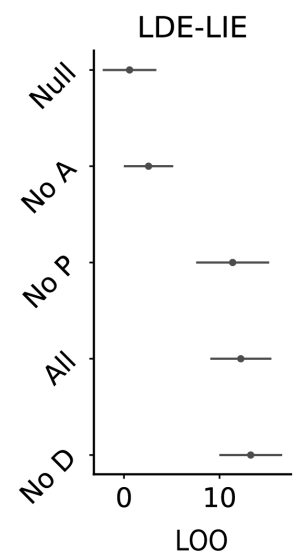

Figure S18: Novel context generalization version 2 initial subject sample results. (A) Distributions across subjects for each performance metric, along with the threshold values. (B) Means of the posterior distributions of each coefficient for each population. (C) Model comparison results for each population. 

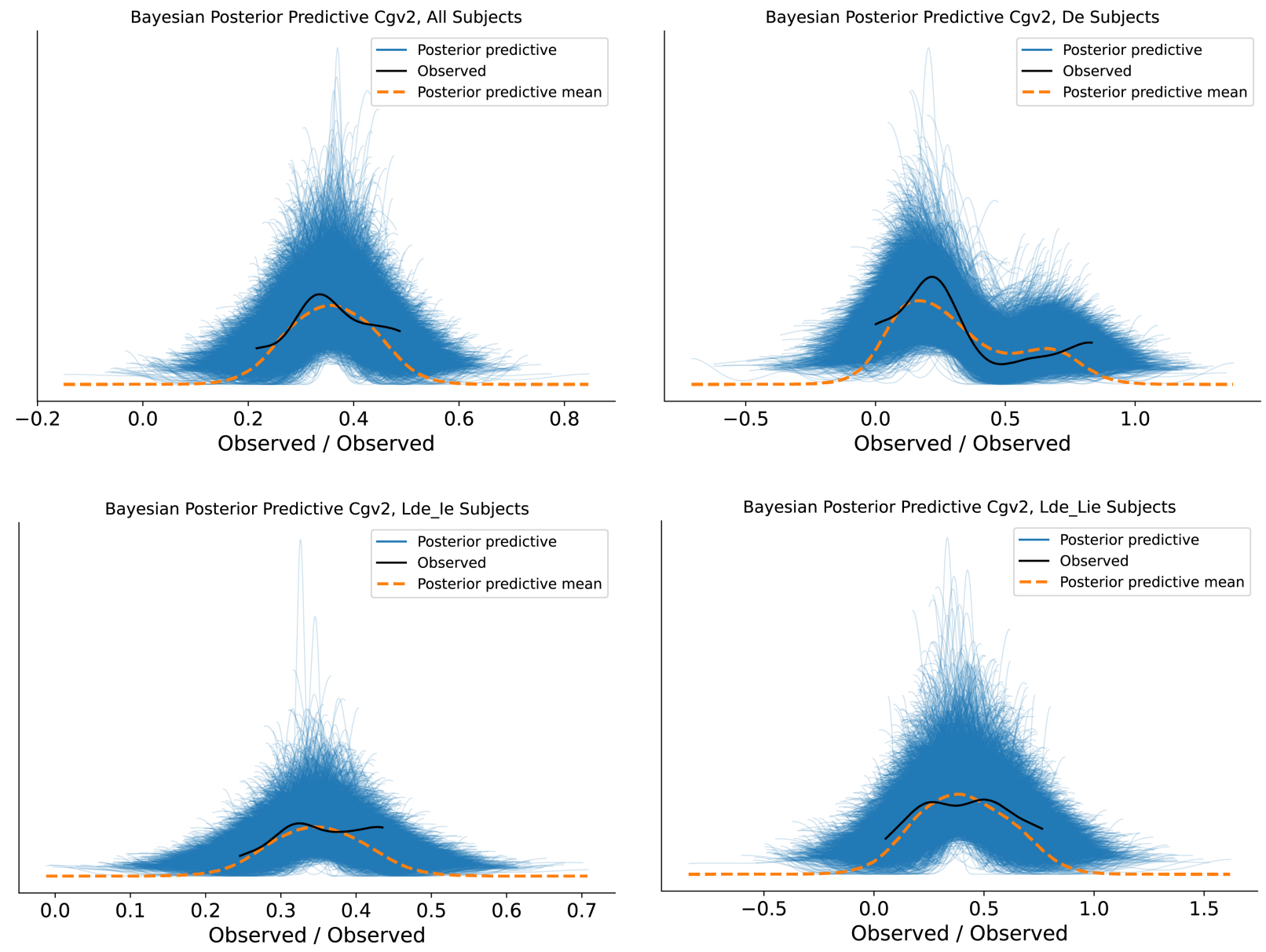

Figure S19: Novel context generalization version 2 posterior predictive distributions by population for the initial sample. 

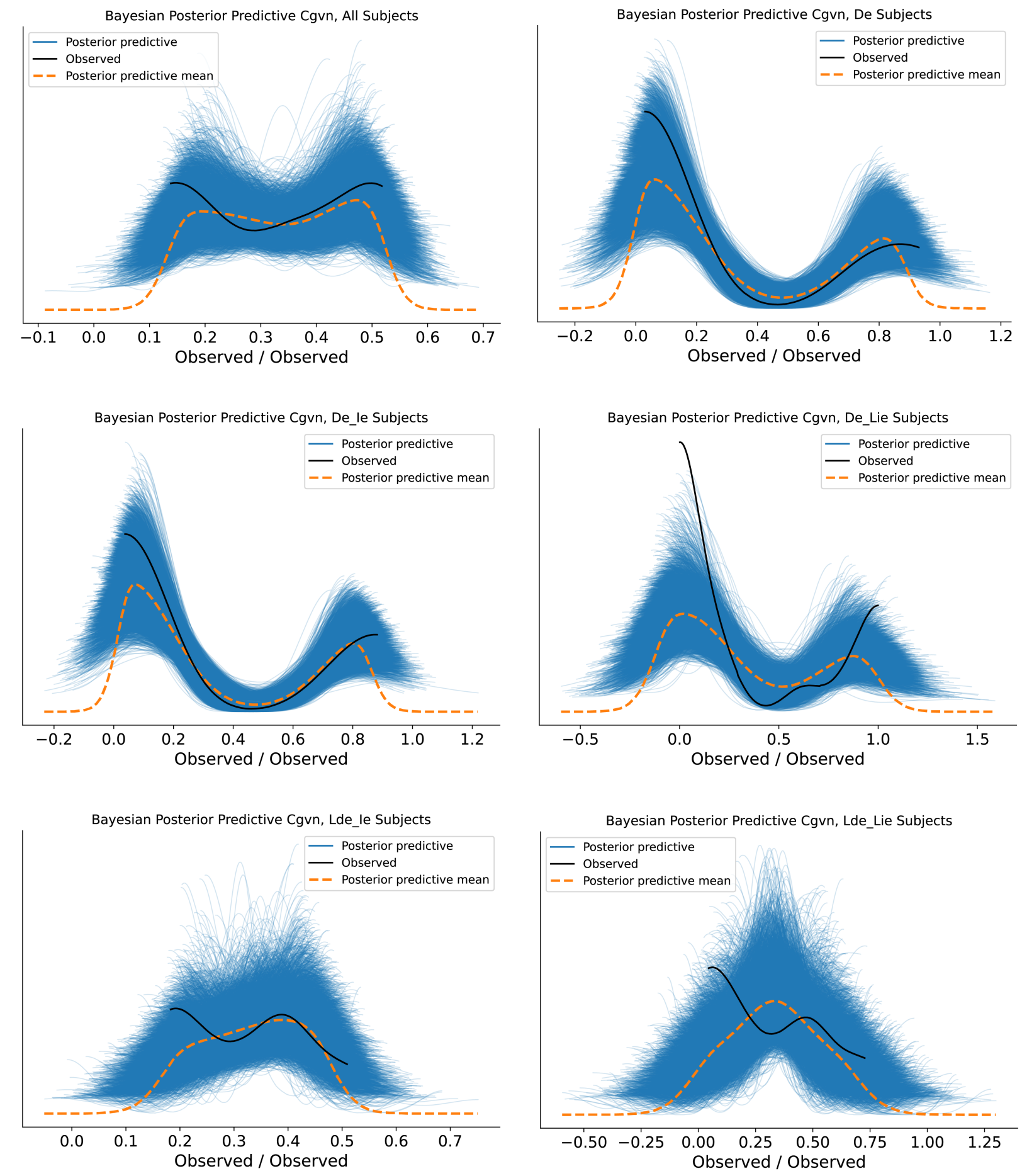

Figure S20: Novel context generalization version 2 posterior predictive distributions by population for the replication sample. 
A

Bayesian Coefficient Distributions
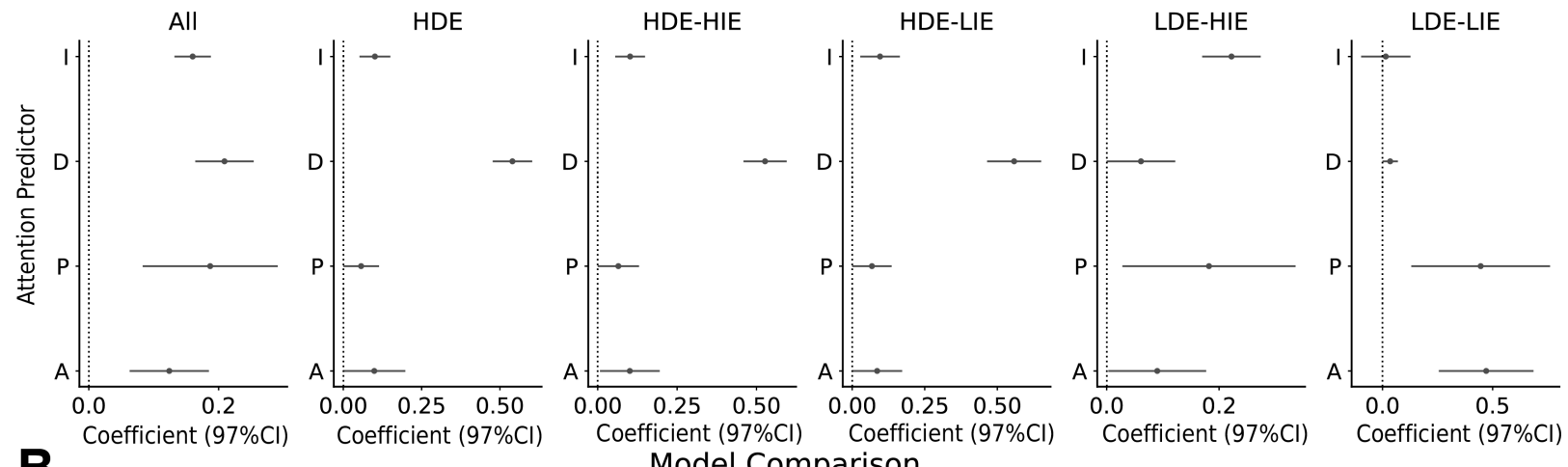

B

Model Comparison
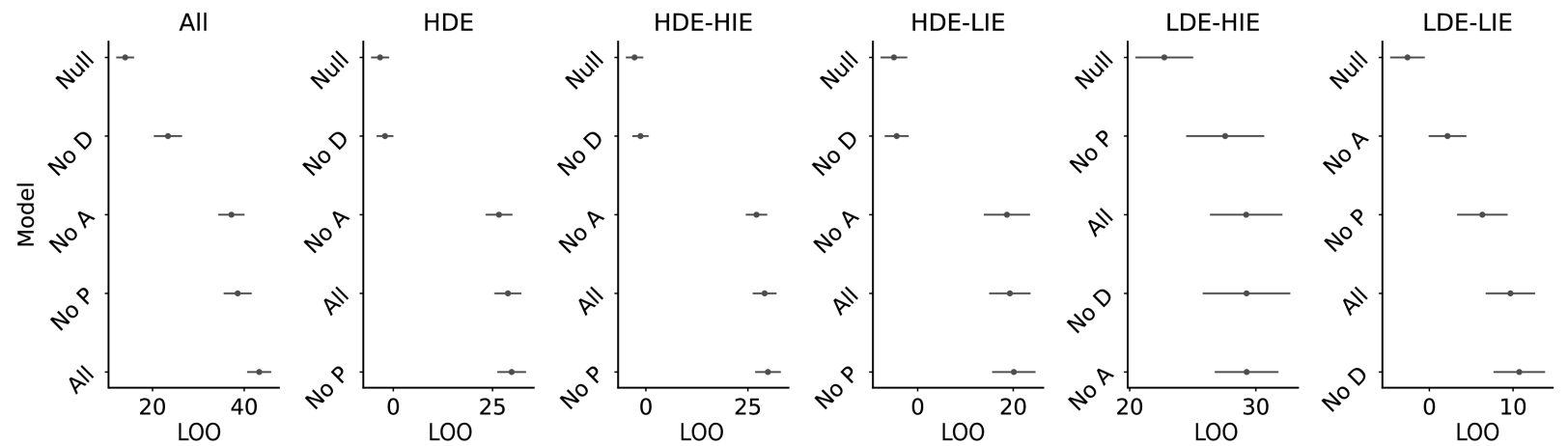

Figure S21: Novel context generalization version 2 replication sample full fit results. (A) Coefficient distribution means and $97 \%$ CI for all populations. (B) Model comparison results for all populations.

A

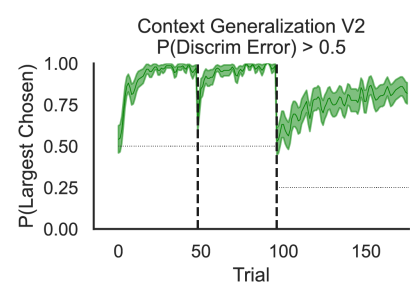

C Initial Generalization Errors, High Discriminative Errors Subpopulations

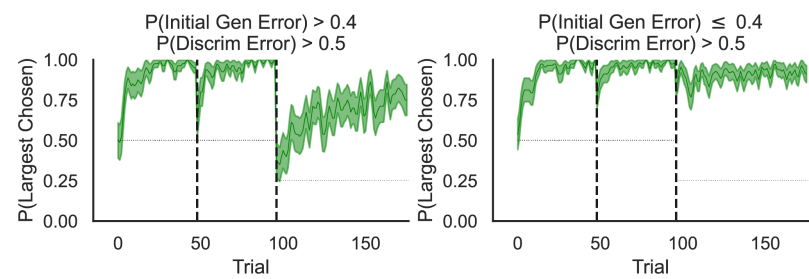

B

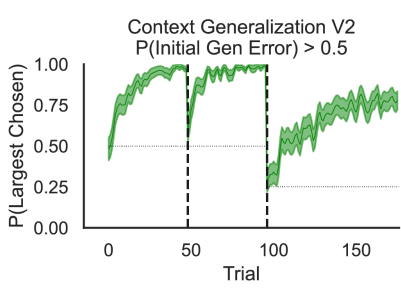

Initial Generalization Errors Subpopulation

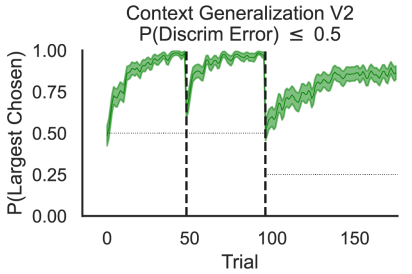

D Initial Generalization Errors, Low Discriminative Errors Subpopulations
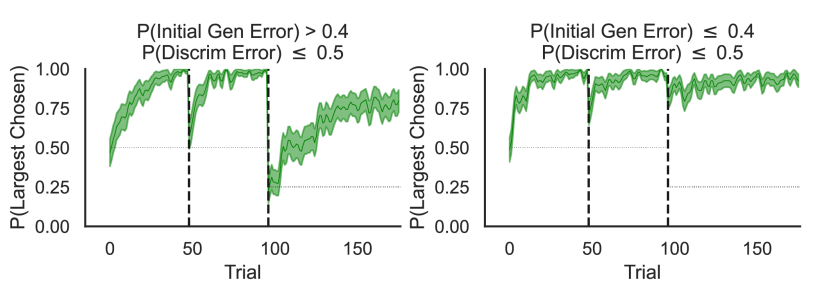

Figure S22: Novel context generalization task version 2, replication subject learning curves divided by threshold values. (A) Subjects separated as above and below the discriminative error threshold. (B) Subjects separated as above or below the initial generalization appearance threshold. (C) Subjects above the discriminative error threshold, separated as above or below the initial generalization appearance threshold. (D) Subjects below the discriminative error threshold, separated as above or below the initial generalization appearance threshold. 

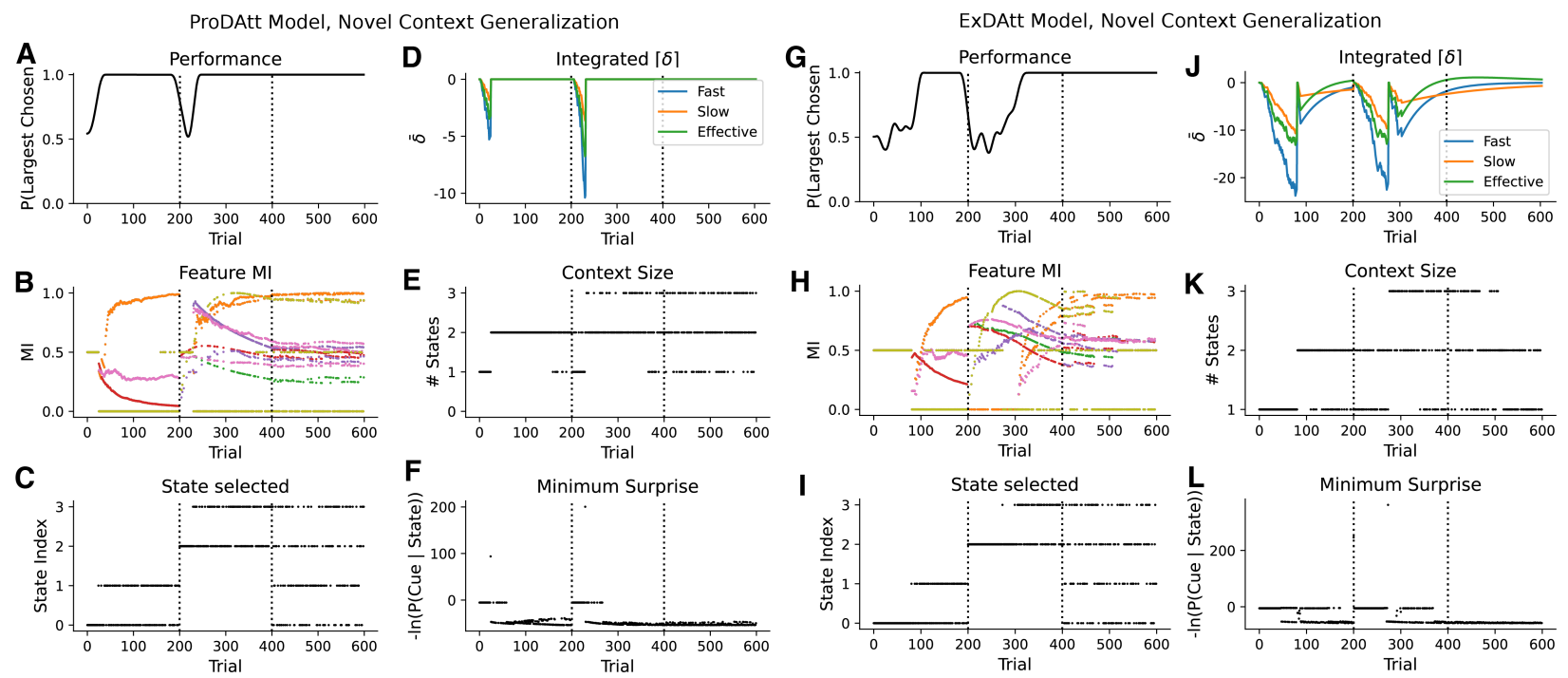

Figure S23: Single agents learn the novel context generalization task version 2. Points are individual trials. (A) a ProDAtt model agent learns the initial representations then transitions to generalization stimuli. (B) The feature mutual information stabilizes as states are created, and adjusts as new examples appear. (C) Four states are learned, of which three are repeatedly used through the transition. (D) The integrated RPE spikes repeatedly until the agent learns a stable set of state representations. (E) The context size fluctuates between trials. (F) The minimum surprise level spikes prior to the creation of new states. (G-L) Same for the ExDAtt model. 

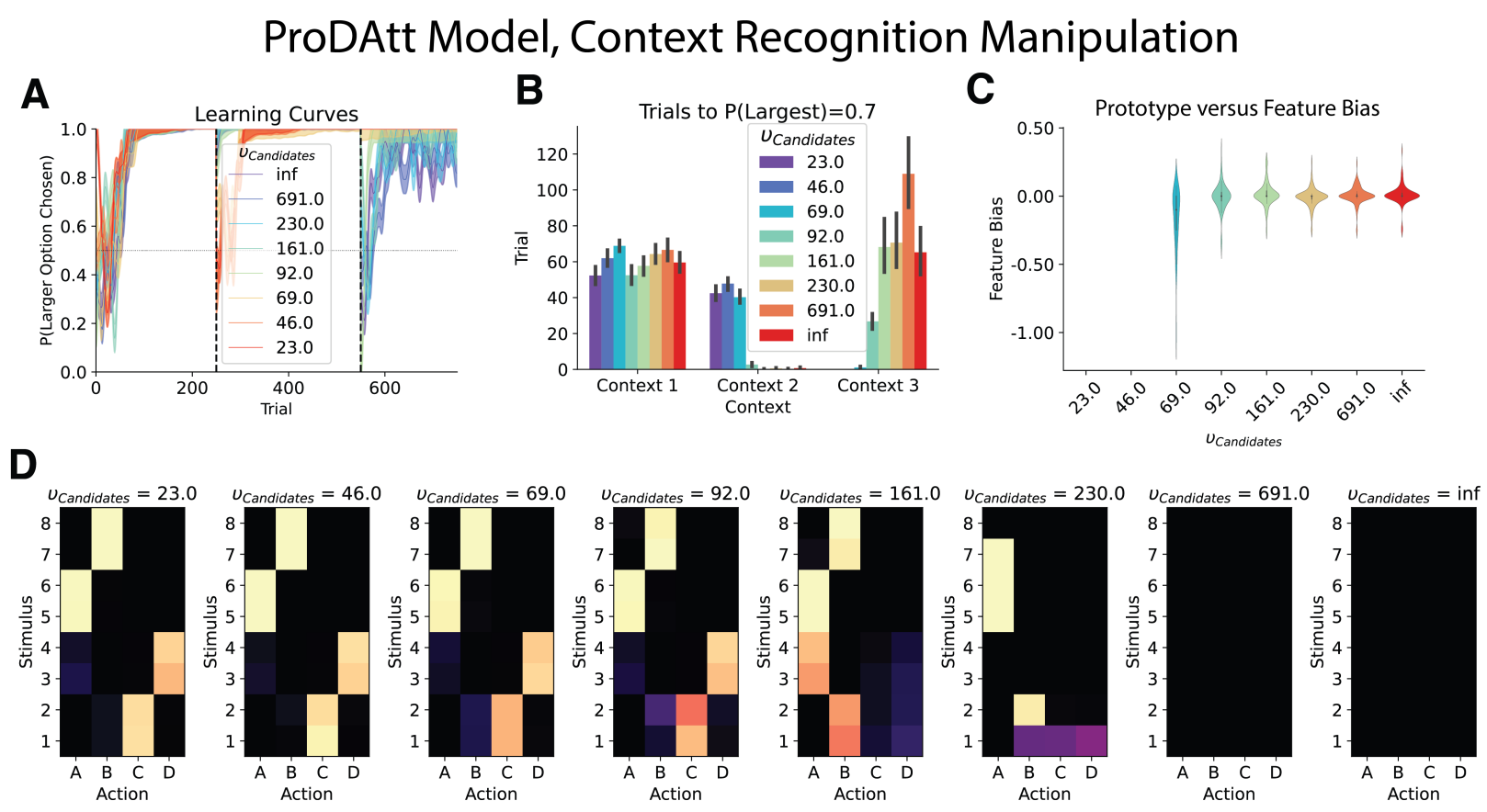

\section{ExDAtt Model, Context Recognition Manipulation}
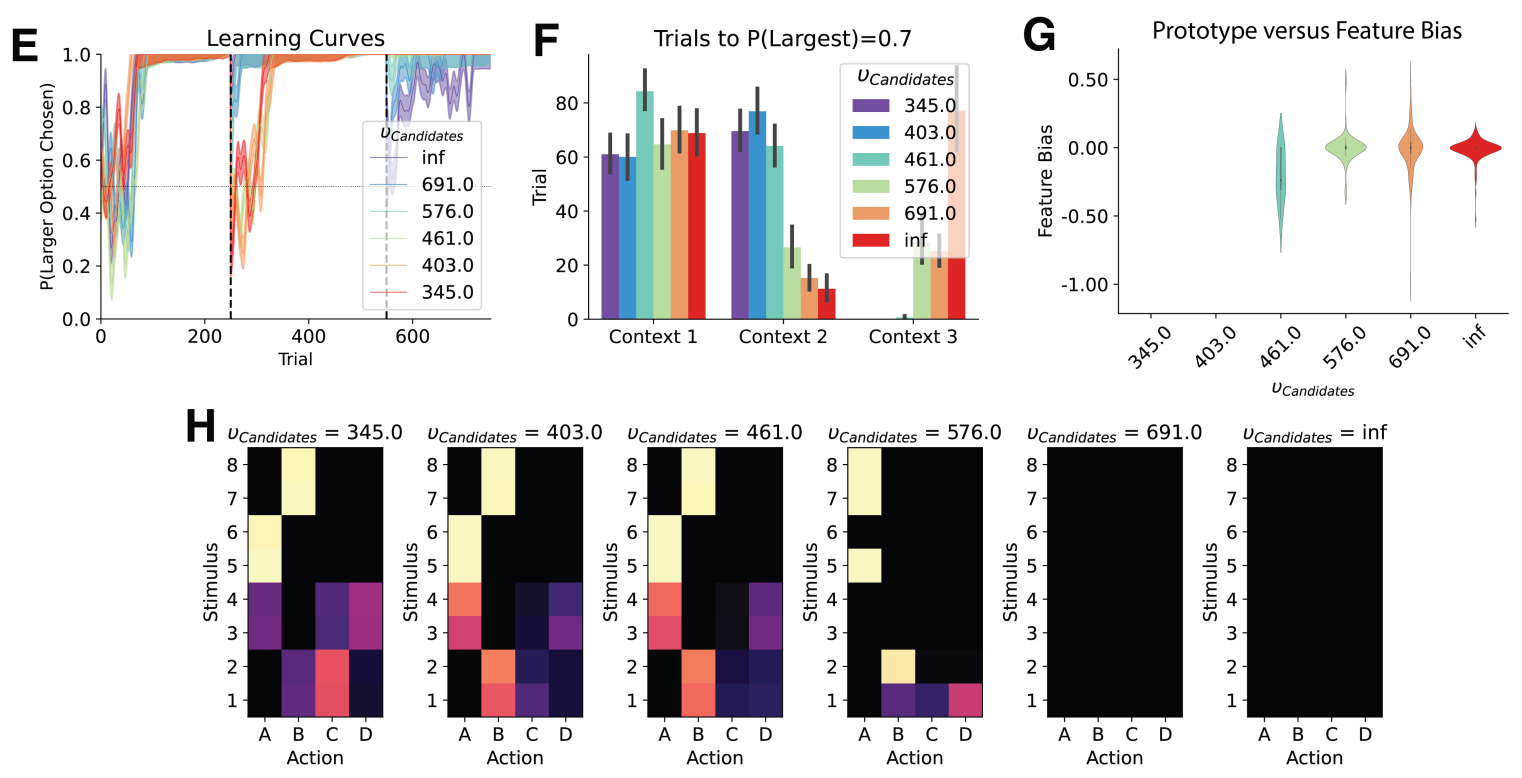

Figure S24: Manipulation of the context inference threshold in the algorithmic model - additional

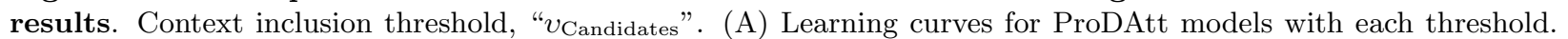
(B) Trials until achieving a threshold of $70 \%$ correct for the ProDAtt model. (C) ProDAtt model feature bias metric as a function of the manipulation. (D) ProDAtt model confusion matrices produced at each threshold. (E-H) same for the ExDAtt model. 


\section{Manipulate Weight Variance}

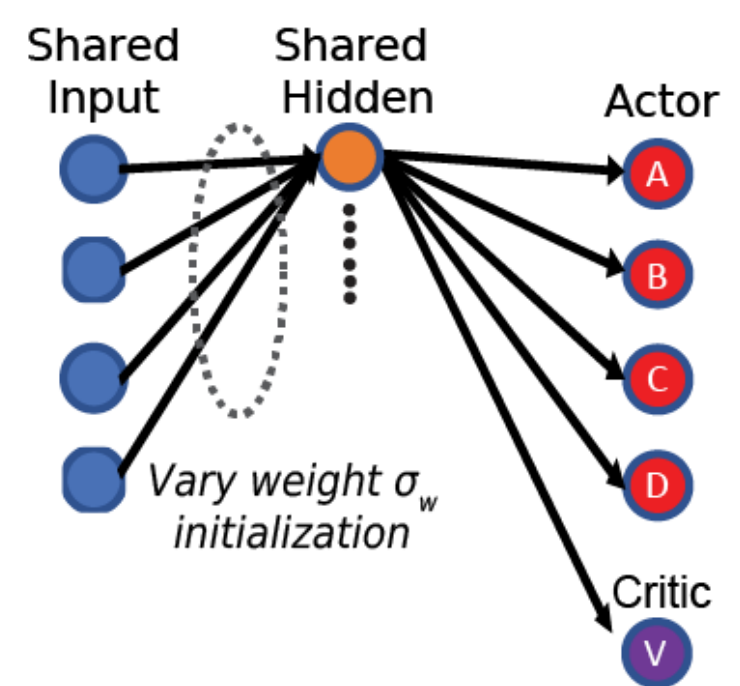

Figure S25: An artificial neural network implementation of actor-critic reinforcement learning (decision boundary learning) where the shared hidden layer weight variance $\left(\sigma_{w}\right)$ is manipulated. 

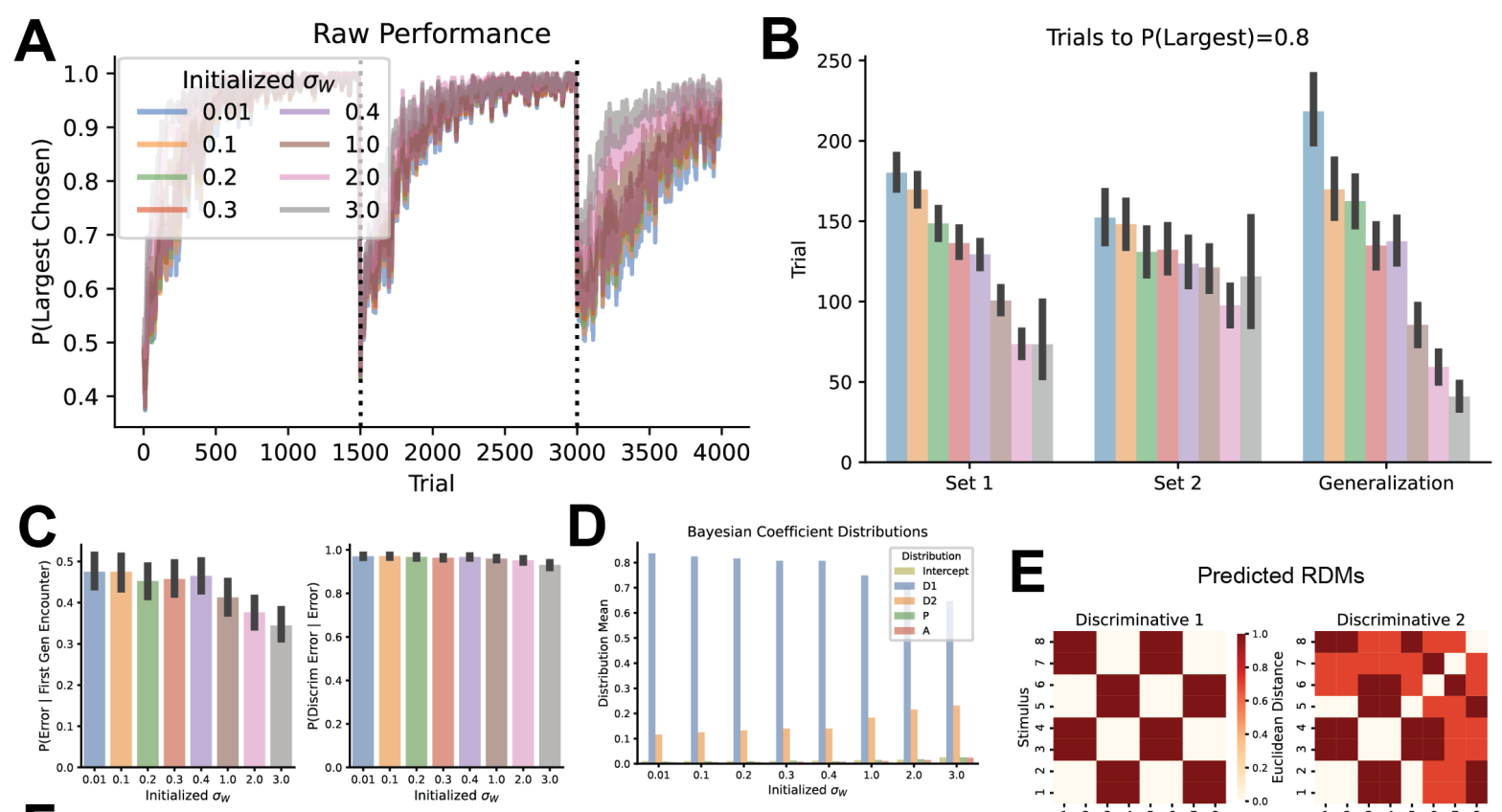

Bayesian Coefficient Distributions
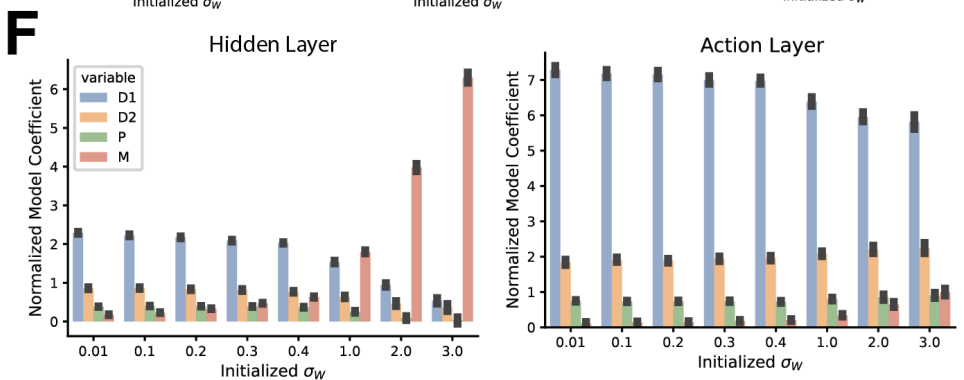

E

Predicted RDMs
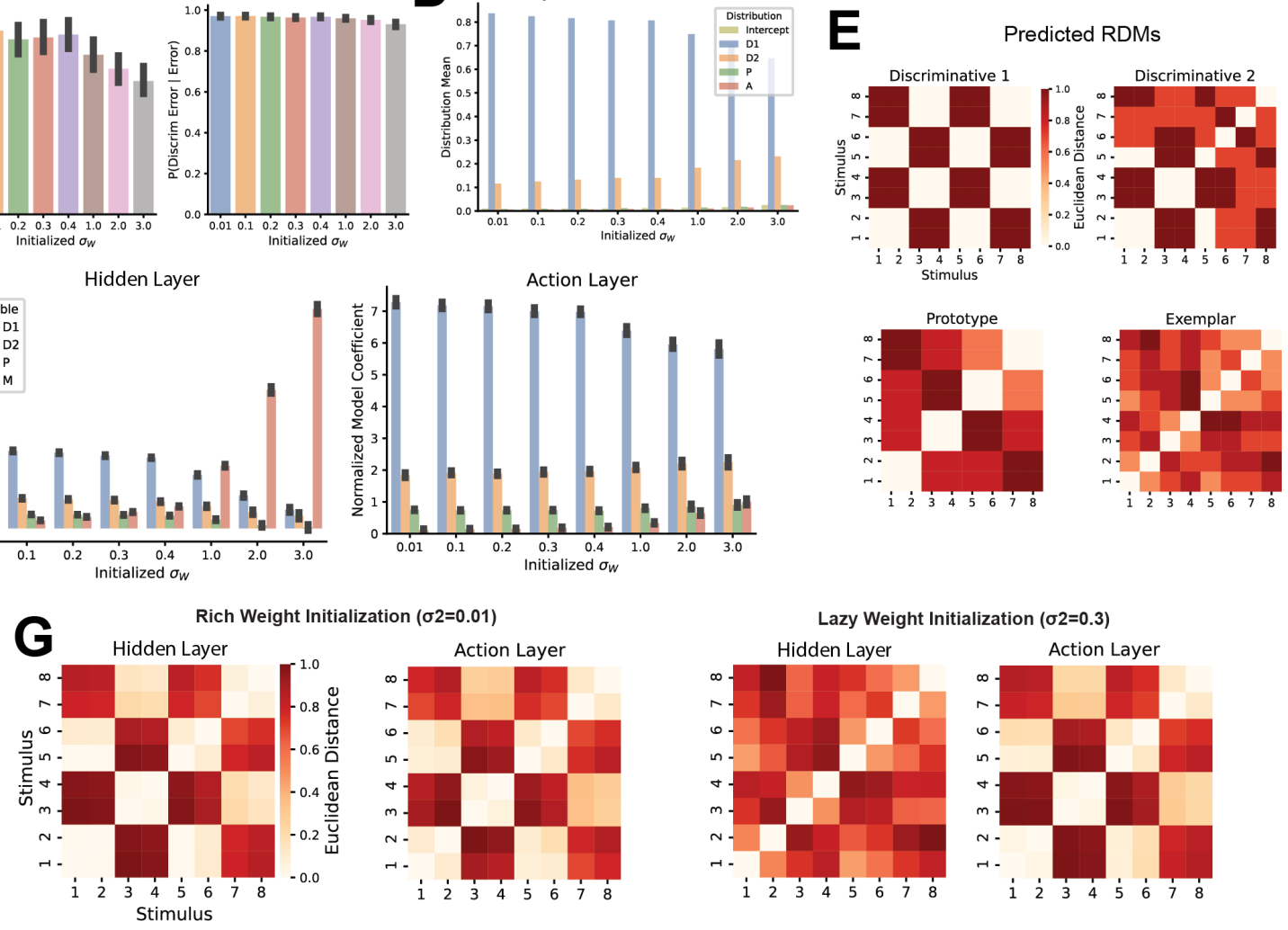

Figure S26: Artificial neural network performing the novel context generalization task version 1 produces discriminative model 1 behavior and discriminative model 1 RDMs in the action layer across hidden layer weight initialization variance levels. Hidden layer weight initialization variance, " $\sigma_{w}$ thresholds." (A) Learning curves for each initialization $\sigma_{w}$. (B) The model learns faster as the initialization $\sigma_{w}$ increases. (C) Increasing the initialization $\sigma_{w}$ causes a slight decrease in initial generalization errors (left), but has no effect on the proportion of discriminative errors (right). (D) Coefficient distributions heavily favored the discriminative 1 idealized attention model. (E) Predicted representational dissimilarity matrices (RDMs) for each idealized model of state formation. (F) Linear regression coefficients by fitting the observed RDMs of the hidden and action layers at each $\sigma_{w}$ initialization show a shift towards an exemplar representation as variance increases in the hidden layer, with a stable bias towards the discriminative representation in the action layer. $(\mathrm{G})$ Example RDS for the hidden and action layers in the right (left) and lazy (right) $\sigma_{w}$ initialization regimes. 

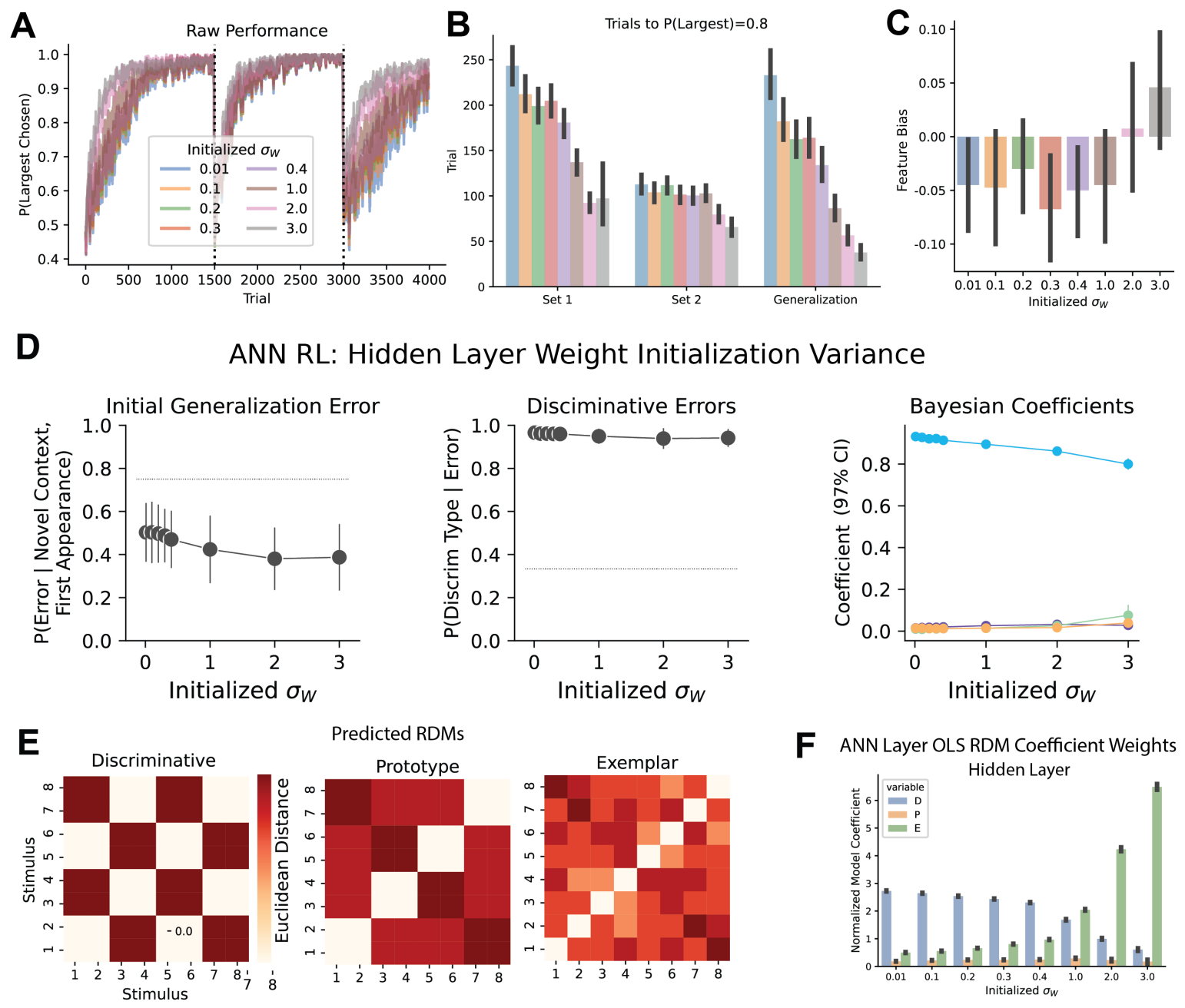

Predicted RDMs
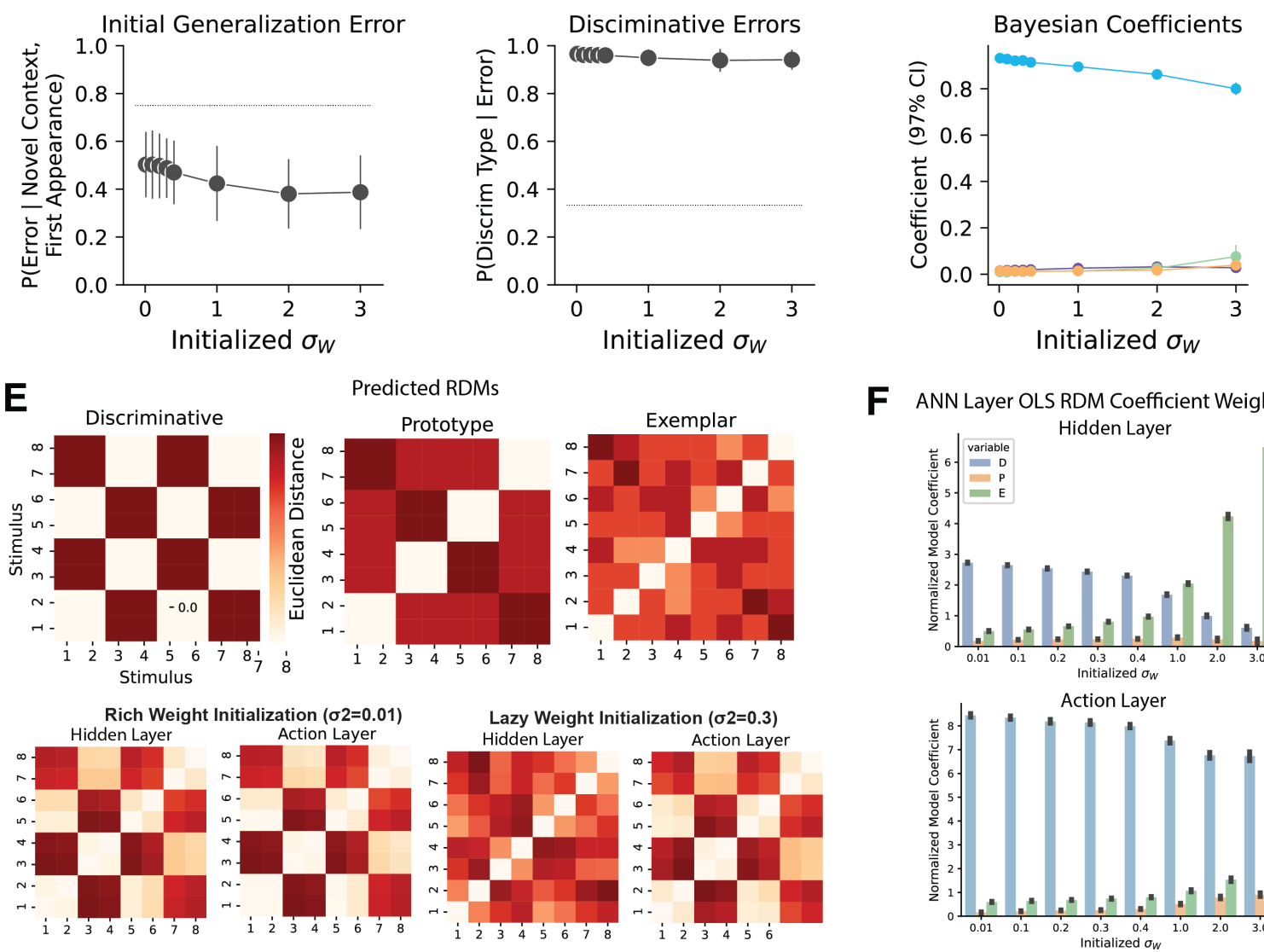

F ANN Layer OLS RDM Coefficient Weights
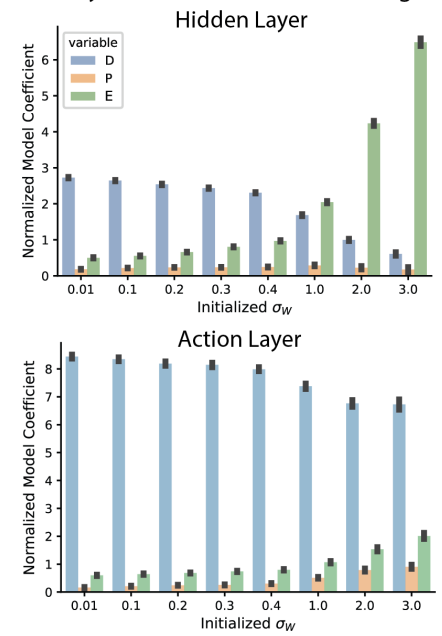

Figure S27: Artificial neural network performing the novel context generalization task version 2 extra. Hidden layer weight initialization variance, " $\sigma_{w}$ ". (A) Learning curves for each initialization $\sigma_{w}$. (B) The model learns faster as the initialization $\sigma_{w}$ increases. (C) As initialization $\sigma_{w}$ increases, there is an increase in the feature-bias metric. (D) Increasing the initialization $\sigma_{w}$ causes a slight decrease in initial generalization errors (left), but has no effect on the proportion of discriminative errors (middle). The coefficient value remains high for the discriminative value across ANN hidden weight $\sigma_{w}$ initializations. Error bars for the error types indicate the standard deviation. Error bars for the Bayesian coefficients represent their 97\% CI. (E) Predicted representational dissimilarity matrices (RDMs) for each idealized model of state formation. (F) Linear regression coefficients by fitting the observed RDMs of the hidden and action layers at each $\sigma_{w}$ initialization show a shift towards an exemplar representation as variance increases in the hidden layer, with a stable bias towards the discriminative representation in the action layer. (G) Example RDS for the hidden and action layers in the right (left) and lazy (right) $\sigma_{w}$ initialization regimes. All error bars represent the standard deviation. 


\section{ProDAtt Model, Random Vs State Estimation Errors}
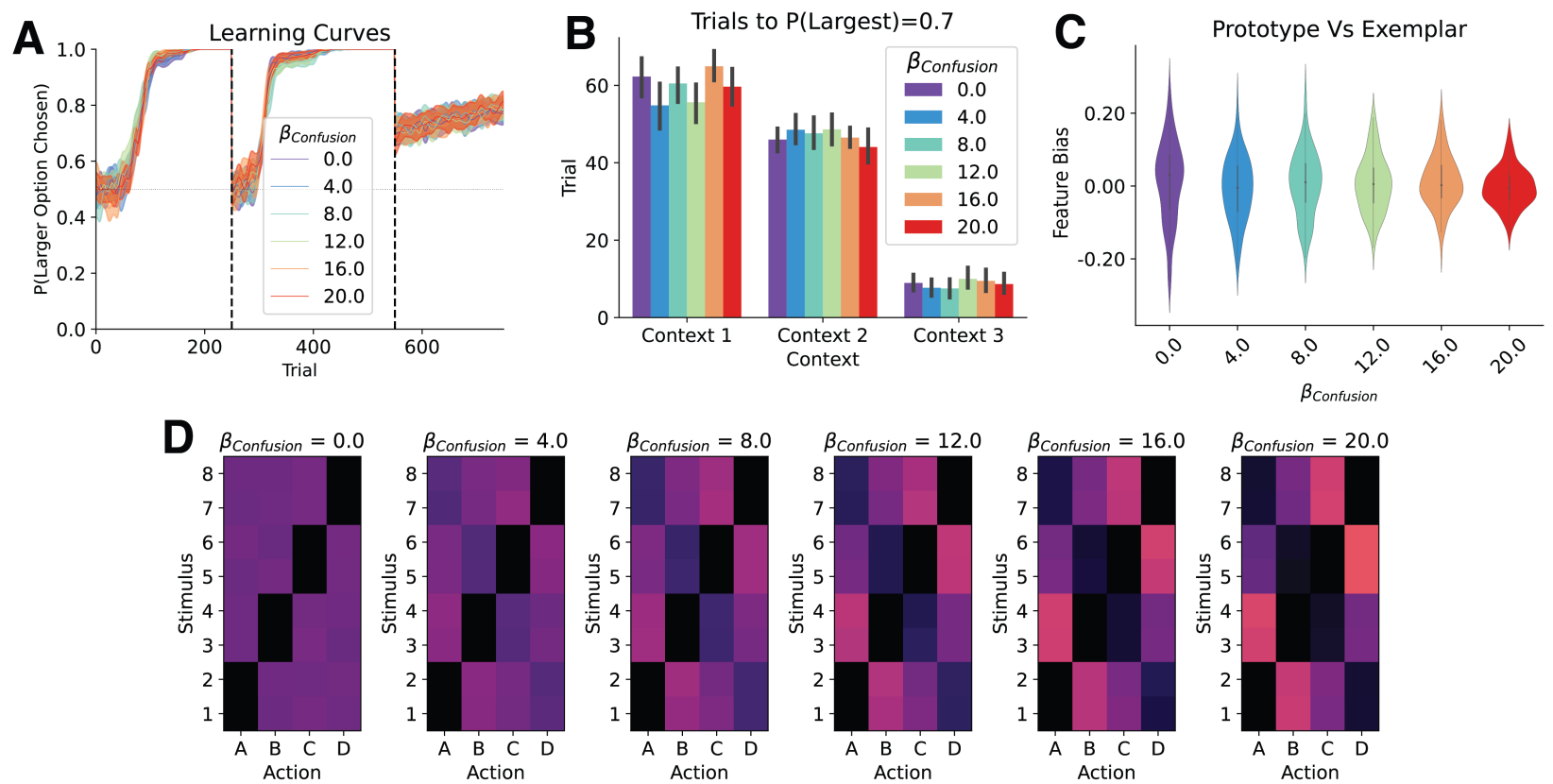

\section{ExDAtt Model, Random Vs State Estimation Errors}
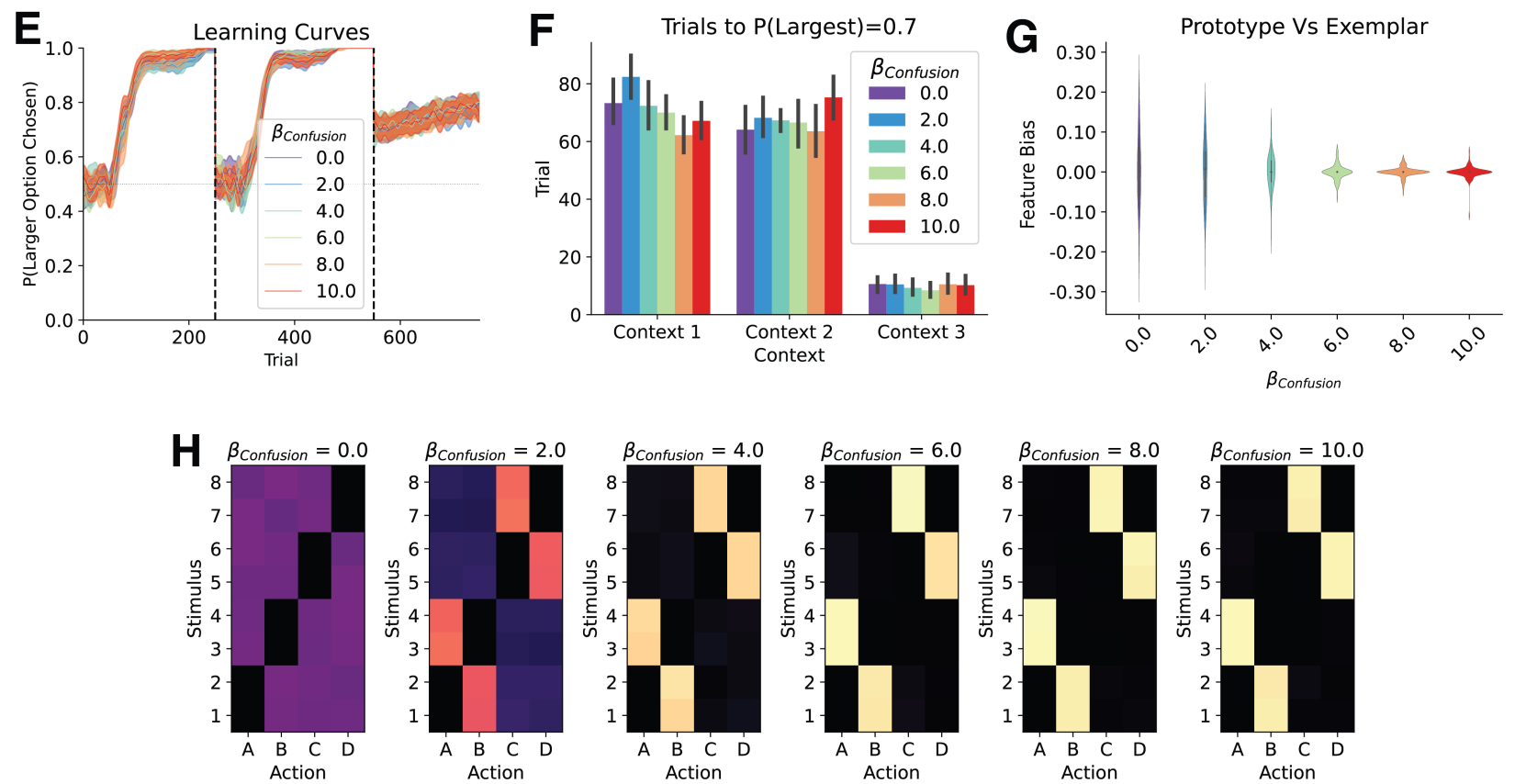

Figure S28: Manipulation of the bias towards random or state estimation errors - additional results. Inverse temperature dictating how much errors are biased by the distance between states versus random, " $\beta_{\text {Confusion." }}$ (A) Learning curves for ProDAtt models with each state estimation error softmax $\beta_{\text {Confusion }}$. (B) Trials until achieving a threshold of $70 \%$ correct for the ProDAtt model. (C) ProDAtt model bias towards exemplar model metric as a function of the state estimation error softmax $\beta$. (D) ProDAtt model confusion matrices produced at each state estimation error softmax $\beta$. (E-H) Same for the ExDAtt model. 


\section{Initial Sample}

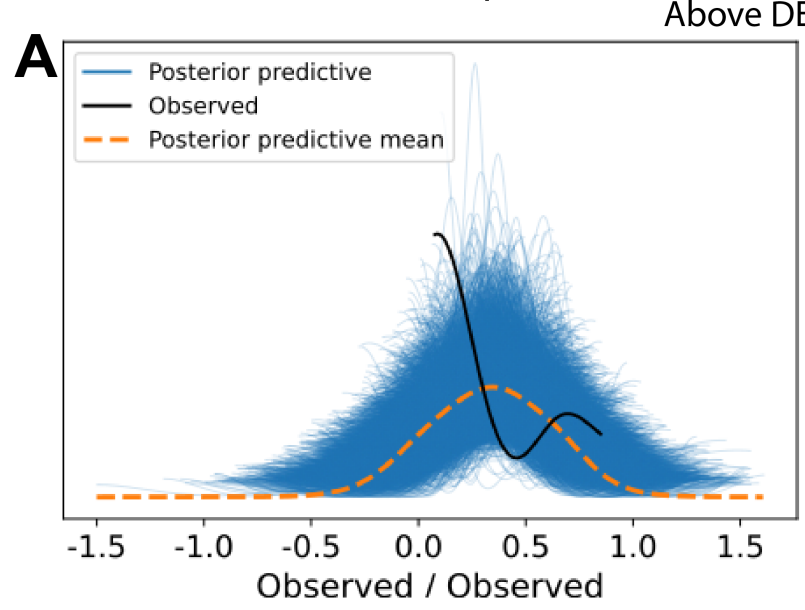

\section{Replication Sample}

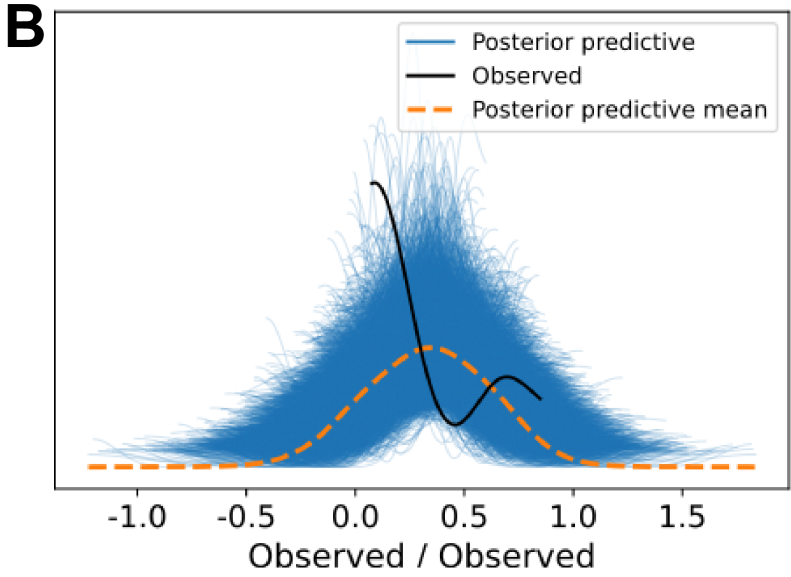

Below DE Threshold, Above IE Threshold
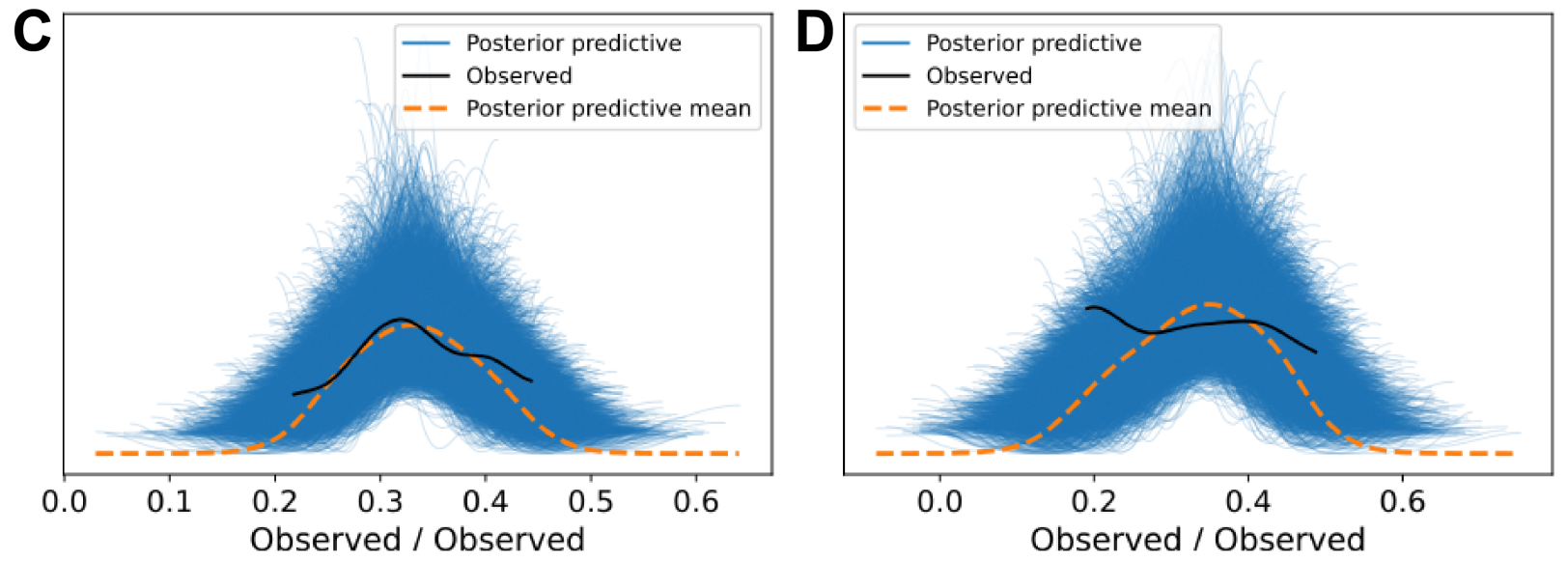

Below DE Threshold, Below IE Threshold
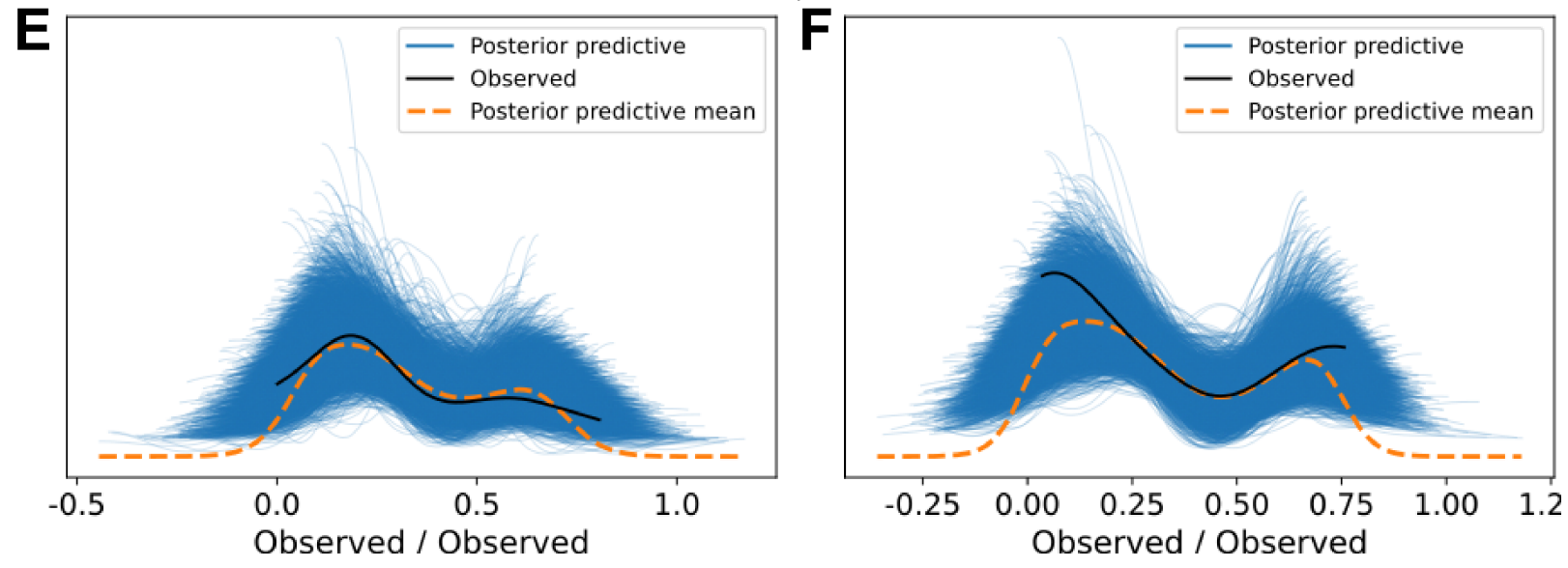

Figure S29: Posterior predictive fits for novel context generalization version 2 initial and replication sample subjects using a model including prototype covariance and state feature bias. 

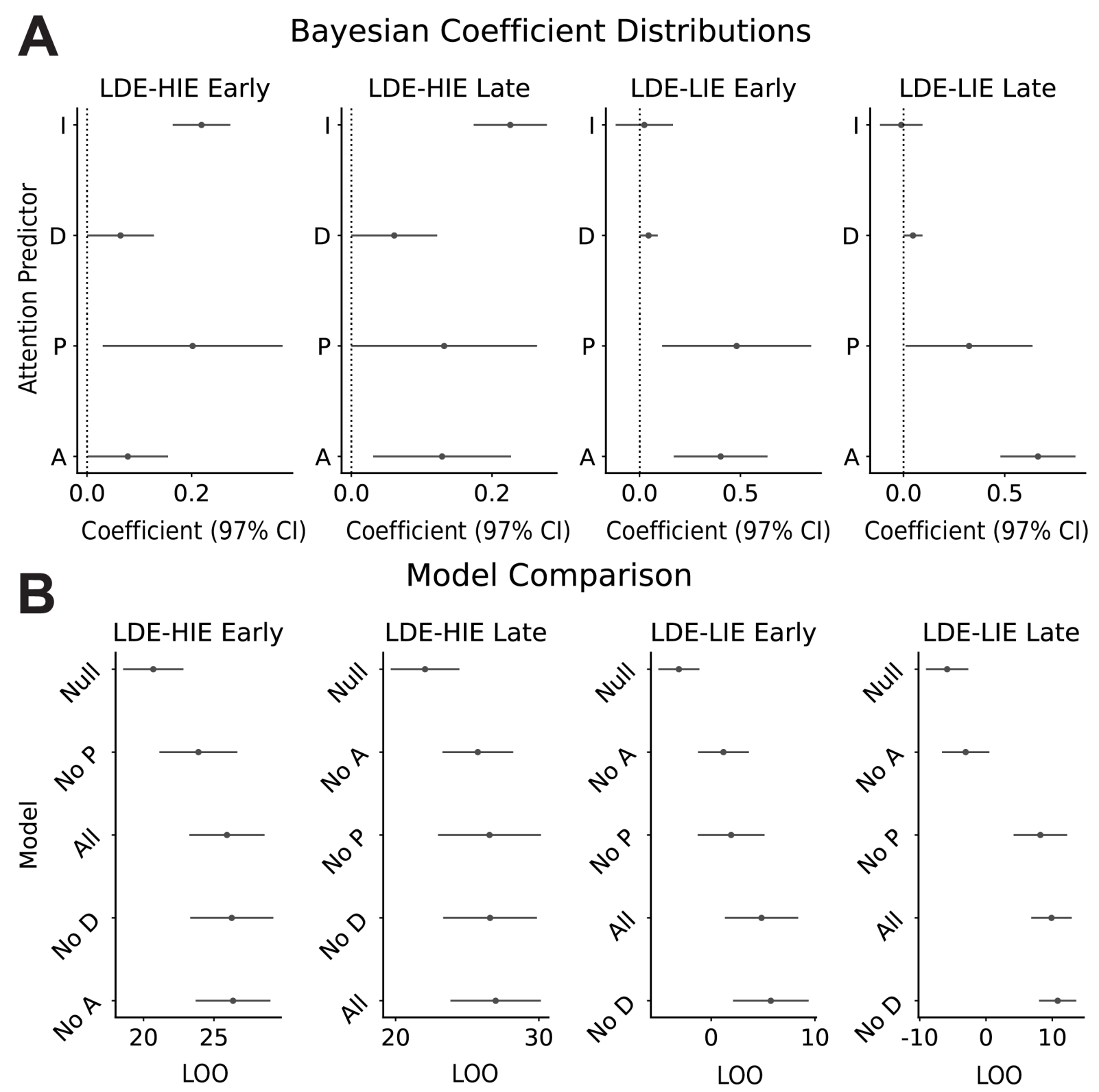

Figure S30: Fitting the confusion matrix separately for the first and second half of the CG $\mathrm{CG}_{2}$ generalization block (A) Fit coefficients for each subpopulation. (B) Model comparison for each subpopulation. 


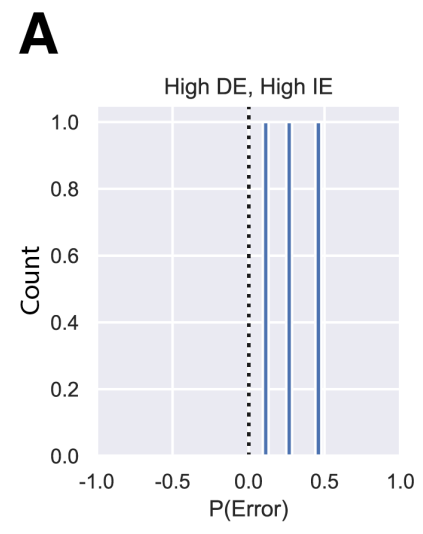

CGV1: P(Error) During Generalization Context

B
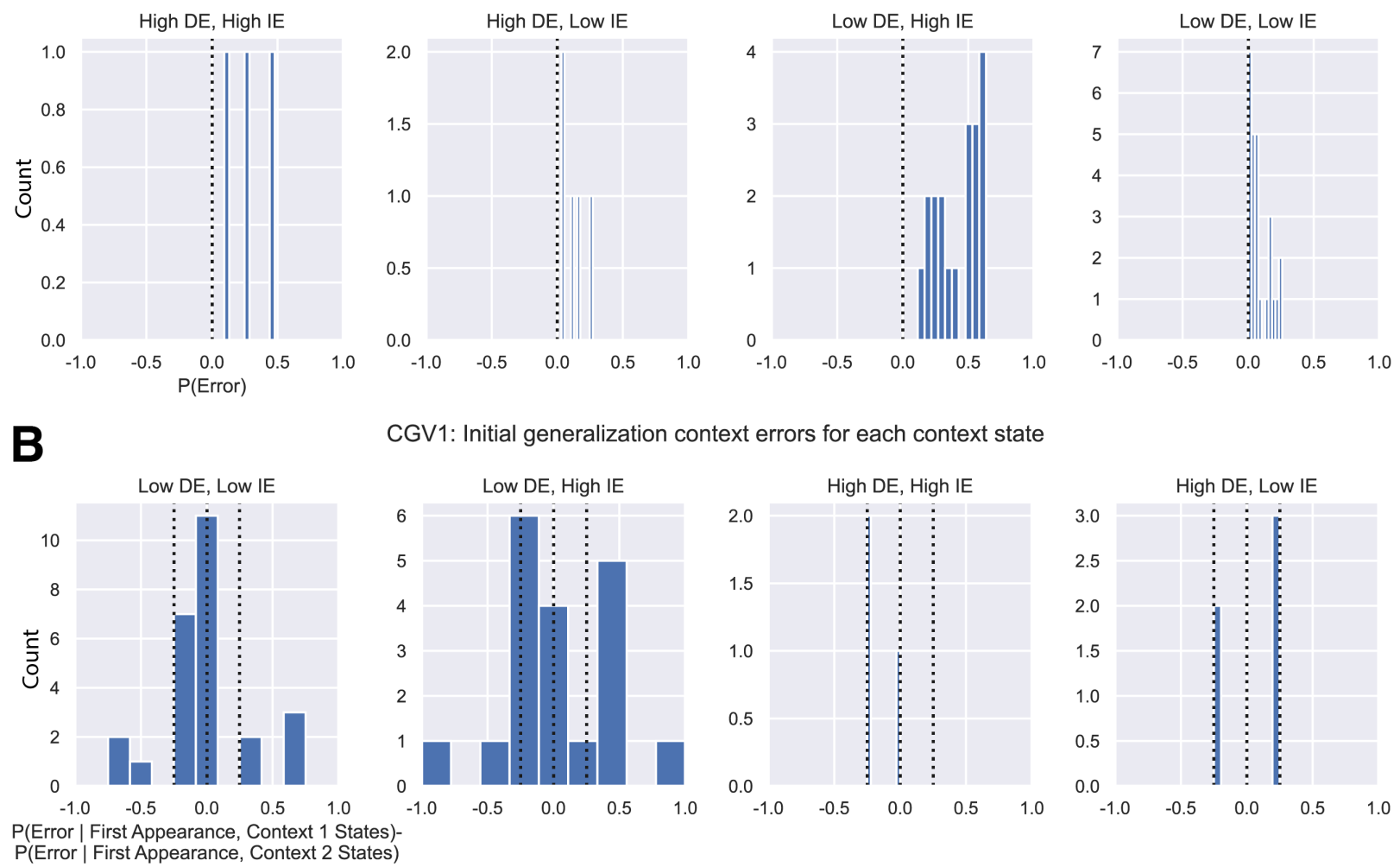

Figure S31: Additional behavioral metrics for context generalization task version 1 subjects. (A) Difference in errors during the generalization between learning context states by subpopulation. (B) Mean errors during generalization context by subpopulation. 
Initial Sample Subjects

CGV2: $P($ Error) During Generalization Context
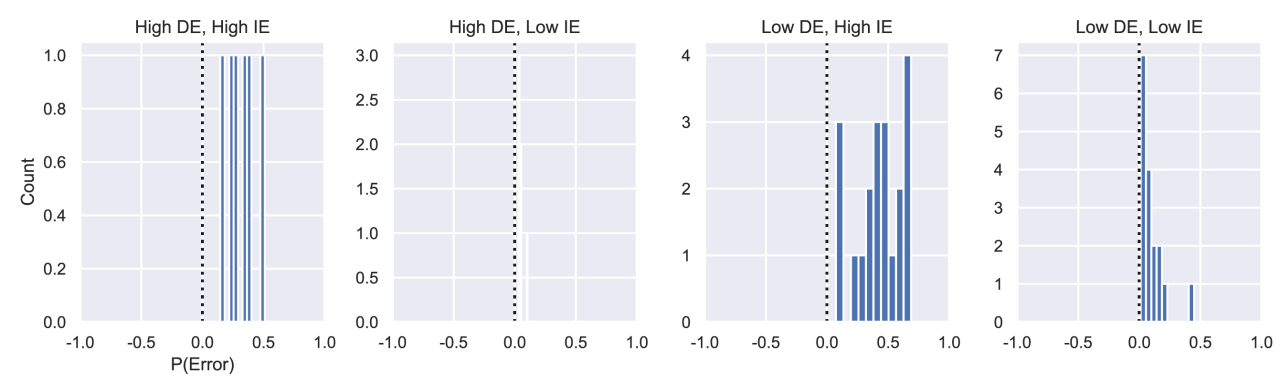

B

CGV2: Initial generalization context errors for each context state
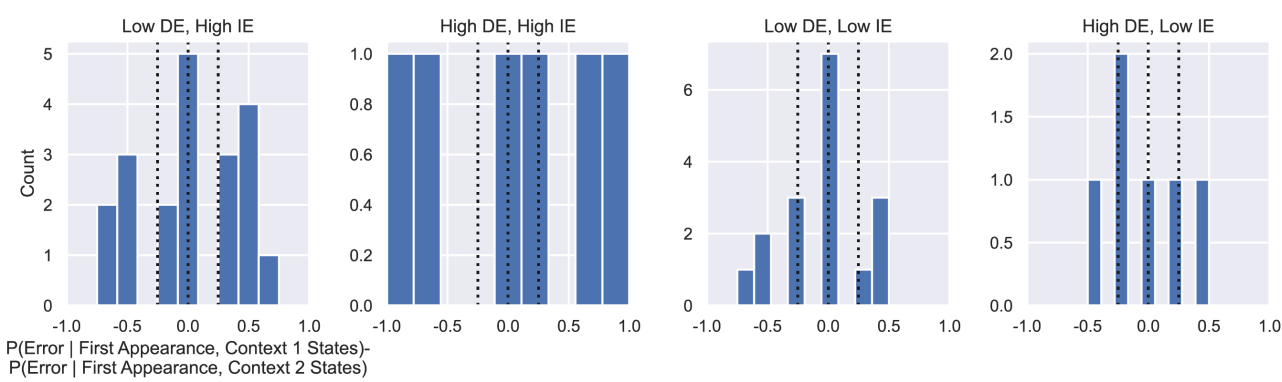

C

Replication Sample Subjects

CGV2: P(Error) During Generalization Context
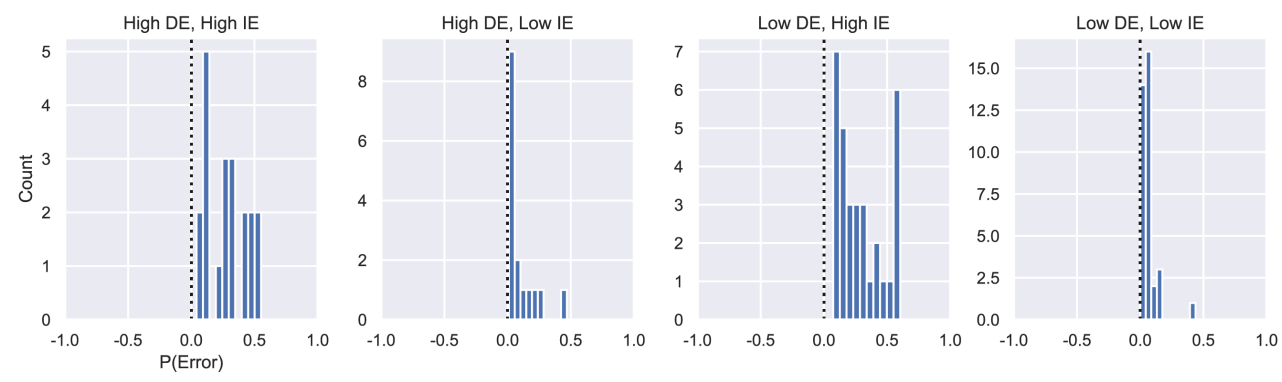

D
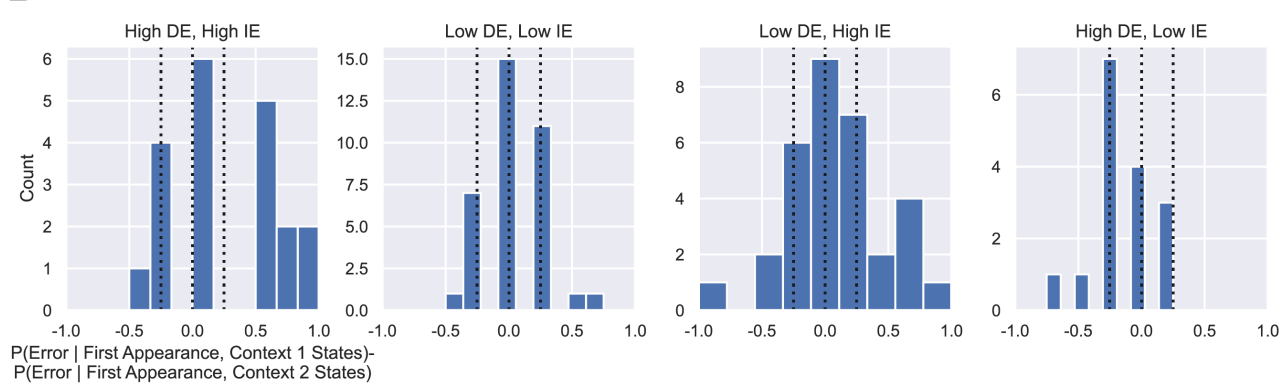

Figure S32: Additional behavioral metrics for context generalization task version 2 subjects. (A) Difference in errors during the generalization between learning context states by subpopulation for the initial sample. (B) Mean errors during generalization context by subpopulation for the initial sample. (C-D), same as (A-B), but for replication sample. 
Supplemental Tables 


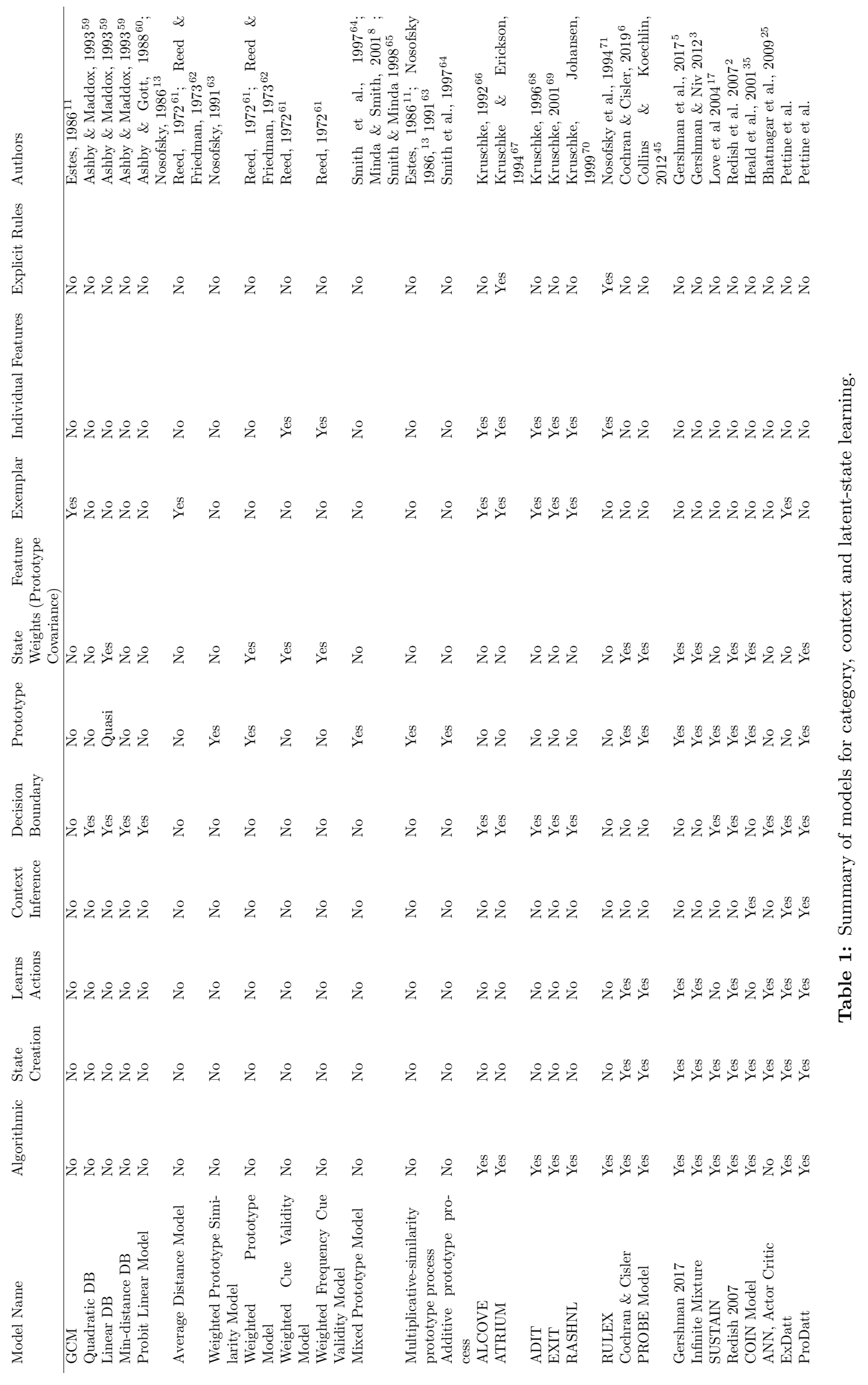




\begin{tabular}{|c|c|c|c|c|c|c|}
\hline Stimulus & Type & Action & Color & Shape & Size & Texture \\
\hline 1 & Learned & A & Orange & Circle & Large & Dots \\
2 & Learned & A & Orange & Square & Large & Dots \\
3 & Learned & B & Magenta & Star & Large & Dots \\
4 & Learned & B & Blue & Star & Large & Dots \\
5 & Learned & C & Magenta & Circle & Large & Dots \\
6 & Learned & C & Magenta & Square & Large & Dots \\
7 & Learned & C & Blue & Circle & Large & Dots \\
8 & Learned & C & Blue & Square & Large & Dots \\
9 & Novel & A & Orange & Circle & Large & Diagonal \\
10 & Novel & A & Orange & Square & Large & Diagonal \\
11 & Novel & B & Magenta & Star & Large & Diagonal \\
12 & Novel & B & Blue & Star & Large & Diagonal \\
13 & Novel & C & Magenta & Circle & Large & Diagonal \\
14 & Novel & C & Magenta & Square & Large & Diagonal \\
15 & Novel & C & Blue & Circle & Large & Diagonal \\
16 & Novel & C & Blue & Square & Large & Diagonal \\
\hline
\end{tabular}

Table 2: Stimulus properties and actions for novel example generalization Experiment 1. 


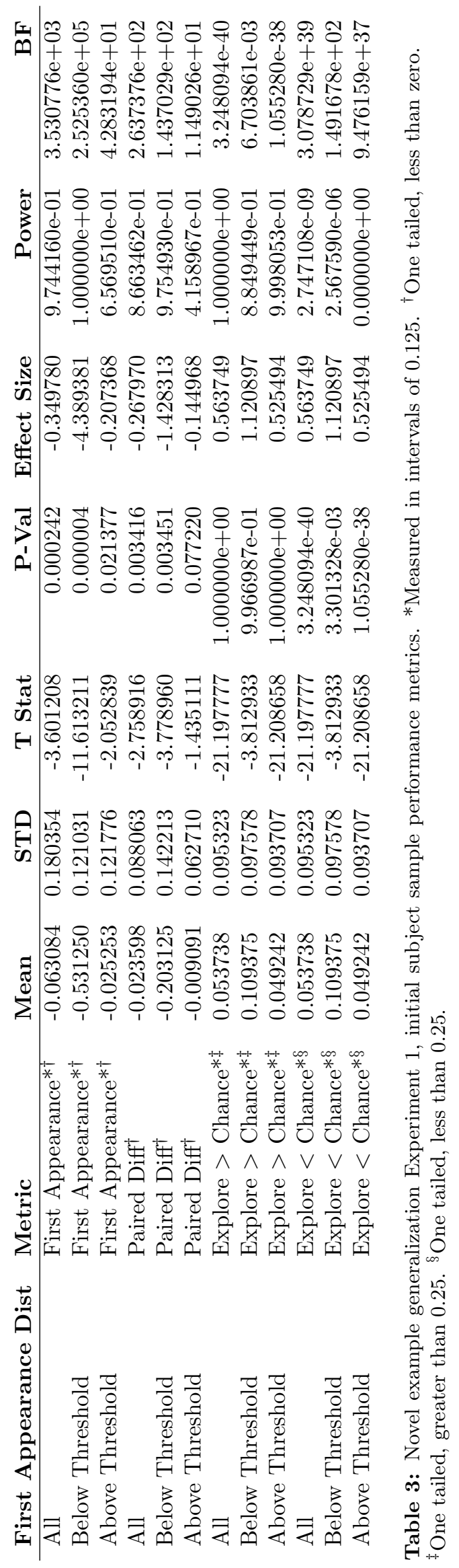




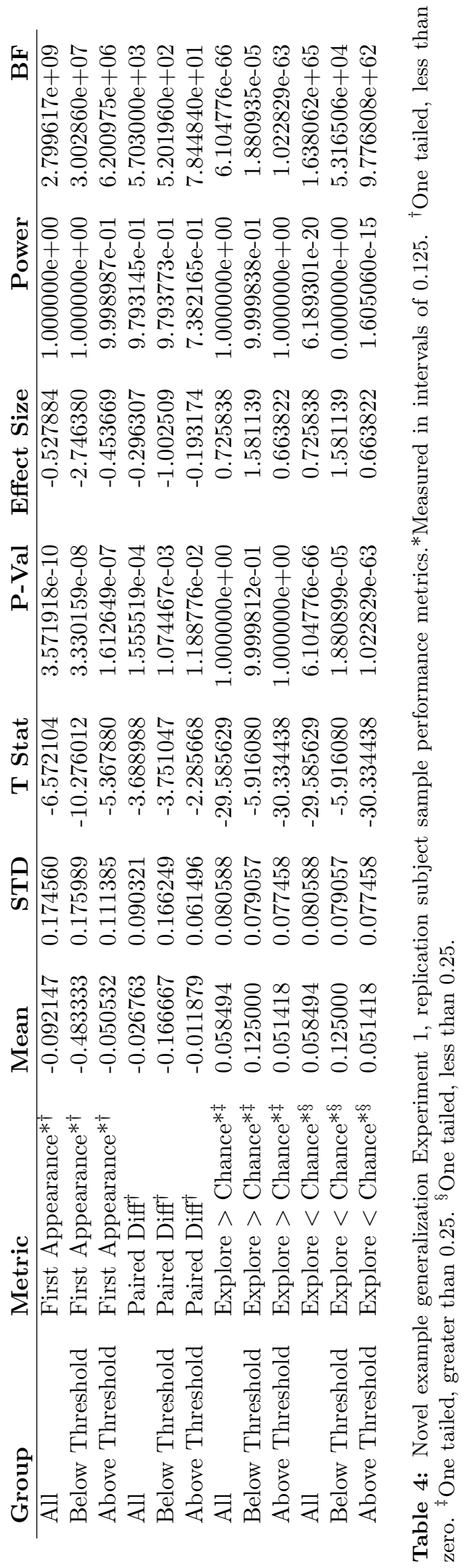




\begin{tabular}{|c|c|c|c|c|c|c|}
\hline Stimulus & Set & Action & Color & Shape & Size & Texture \\
\hline 1 & 1 & A & Magenta & Circle & Large & Dots \\
2 & 1 & A & Magenta & Circle & Small & Dots \\
3 & 1 & B & Orange & Square & Large & Dots \\
4 & 1 & B & Orange & Square & Small & Dots \\
5 & 2 & C & Magenta & Circle & Large & Diagonal \\
6 & 2 & C & Blue & Circle & Large & Diagonal \\
7 & 2 & D & Magenta & Square & Large & Diagonal \\
8 & 2 & D & Blue & Square & Large & Diagonal \\
\hline
\end{tabular}

Table 5: Stimulus properties and actions for novel context generalization Experiment 2. 


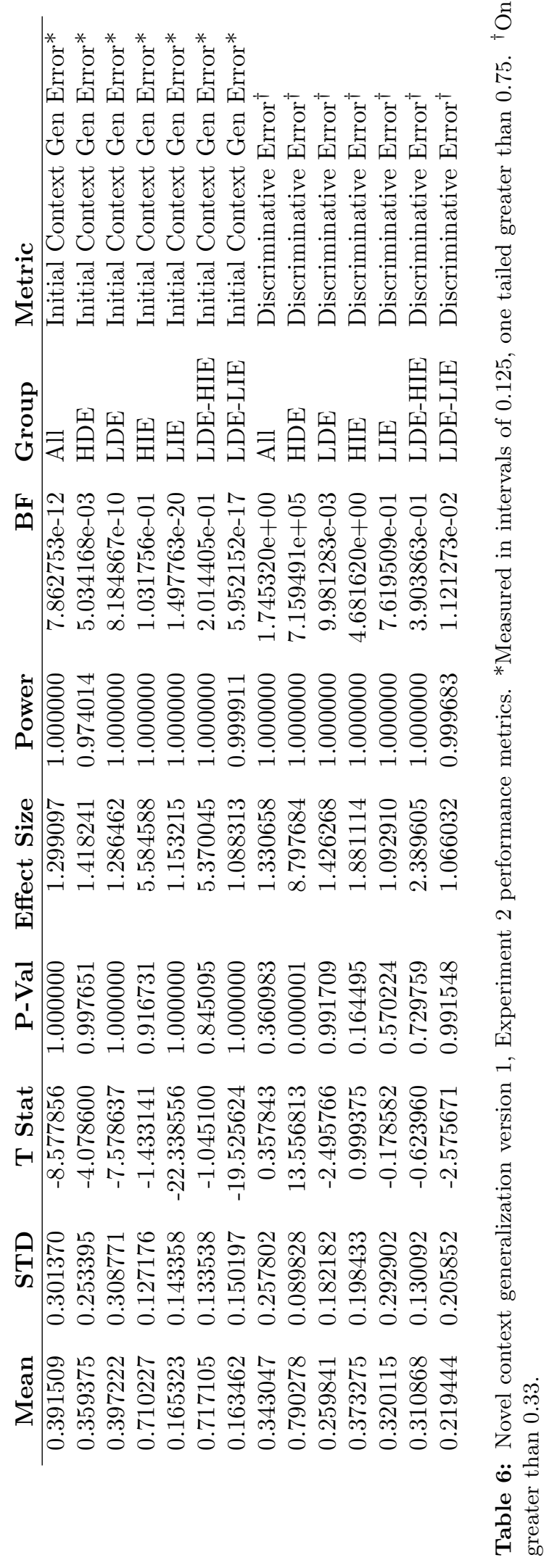




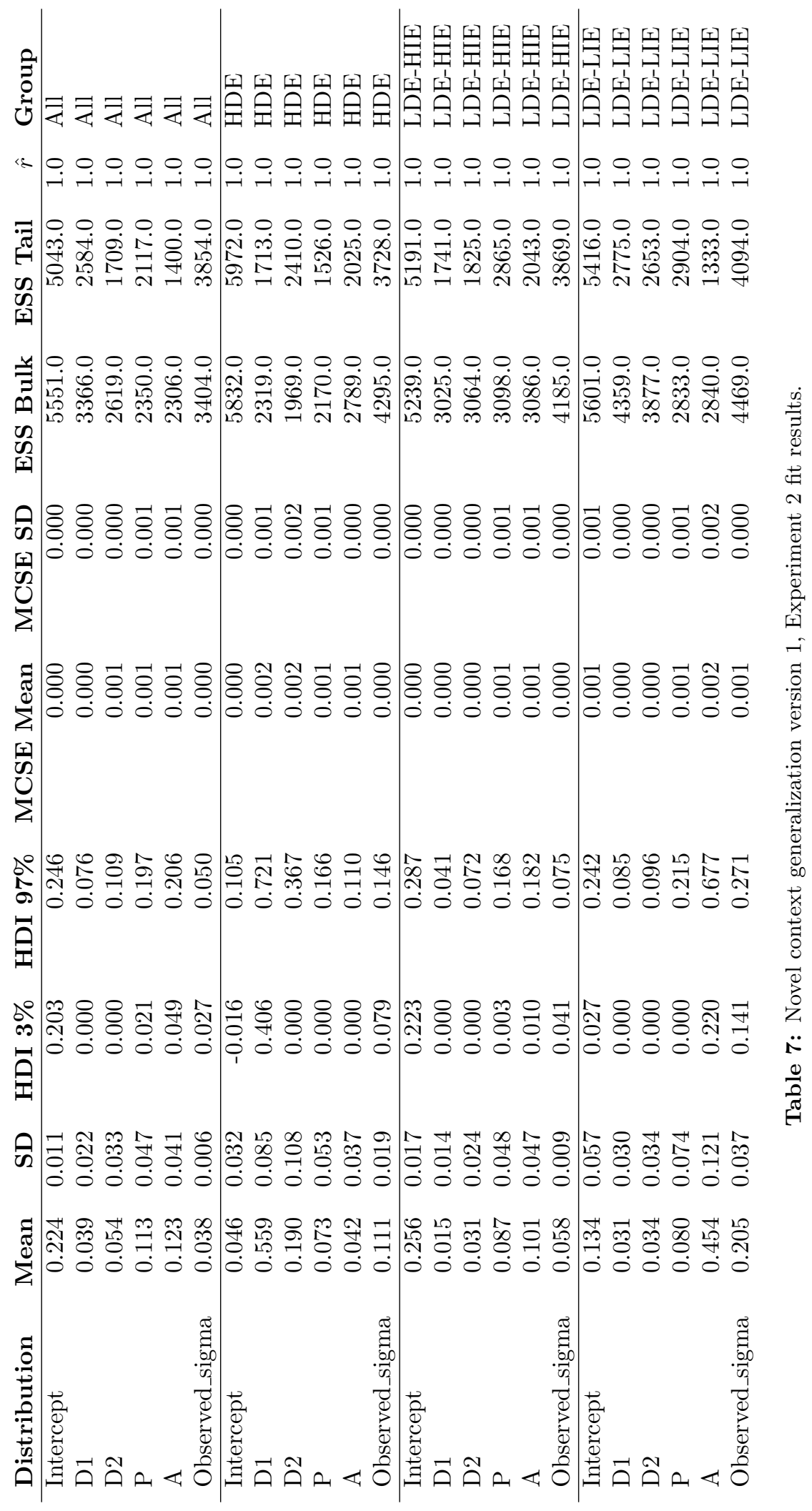




\begin{tabular}{lrrrl} 
Model & Rank & LOO & SE & Group \\
\hline All & 0 & 42.950327 & 2.933542 & All \\
No D2 & 1 & 42.741244 & 2.947772 & All \\
No D1 & 2 & 42.483620 & 3.219175 & All \\
No P & 3 & 40.462877 & 2.870937 & All \\
No A & 4 & 39.872918 & 2.611349 & All \\
Null & 5 & 16.458233 & 2.398786 & All \\
\hline No A & 0 & 16.979885 & 3.855598 & HDE \\
No P & 1 & 16.524611 & 3.467195 & HDE \\
All & 2 & 16.488424 & 3.667512 & HDE \\
No D2 & 3 & 15.387274 & 4.492738 & HDE \\
No D1 & 4 & 4.414764 & 4.877567 & HDE \\
Null & 5 & -10.753678 & 2.310211 & HDE \\
\hline No D1 & 0 & 33.967040 & 2.393504 & LDE-HIE \\
No D2 & 1 & 33.426689 & 2.278600 & LDE-HIE \\
All & 2 & 33.334222 & 2.249724 & LDE-HIE \\
No P & 3 & 32.310336 & 2.437761 & LDE-HIE \\
No A & 4 & 32.274036 & 2.490349 & LDE-HIE \\
Null & 5 & 20.988144 & 3.513232 & LDE-HIE \\
\hline No P & 0 & 3.577170 & 4.837005 & LDE-LIE \\
No D1 & 1 & 3.525676 & 4.571162 & LDE-LIE \\
No D2 & 2 & 3.494118 & 4.601122 & LDE-LIE \\
All & 3 & 2.589935 & 4.427715 & LDE-LIE \\
No A & 4 & -3.139531 & 3.530814 & LDE-LIE \\
Null & 5 & -4.462201 & 2.679690 & LDE-LIE \\
& & & &
\end{tabular}

Table 8: Novel context generalization task version 1, Experiment 2 model comparison.

\begin{tabular}{|c|c|c|c|c|c|c|}
\hline Stimulus & Set & Action & Color & Shape & Size & Texture \\
\hline 1 & 1 & A & Orange & Circle & Large & Dots \\
2 & 1 & A & Magenta & Circle & Small & Dots \\
3 & 1 & B & Magenta & Square & Large & Dots \\
4 & 1 & B & Orange & Square & Large & Dots \\
5 & 2 & C & Magenta & Circle & Large & Diagonal \\
6 & 2 & C & Blue & Circle & Large & Diagonal \\
7 & 2 & D & Magenta & Square & Small & Diagonal \\
8 & 2 & D & Blue & Square & Large & Diagonal \\
\hline
\end{tabular}

Table 9: Stimulus properties and actions for novel context generalization version 2, Experiment 3. 


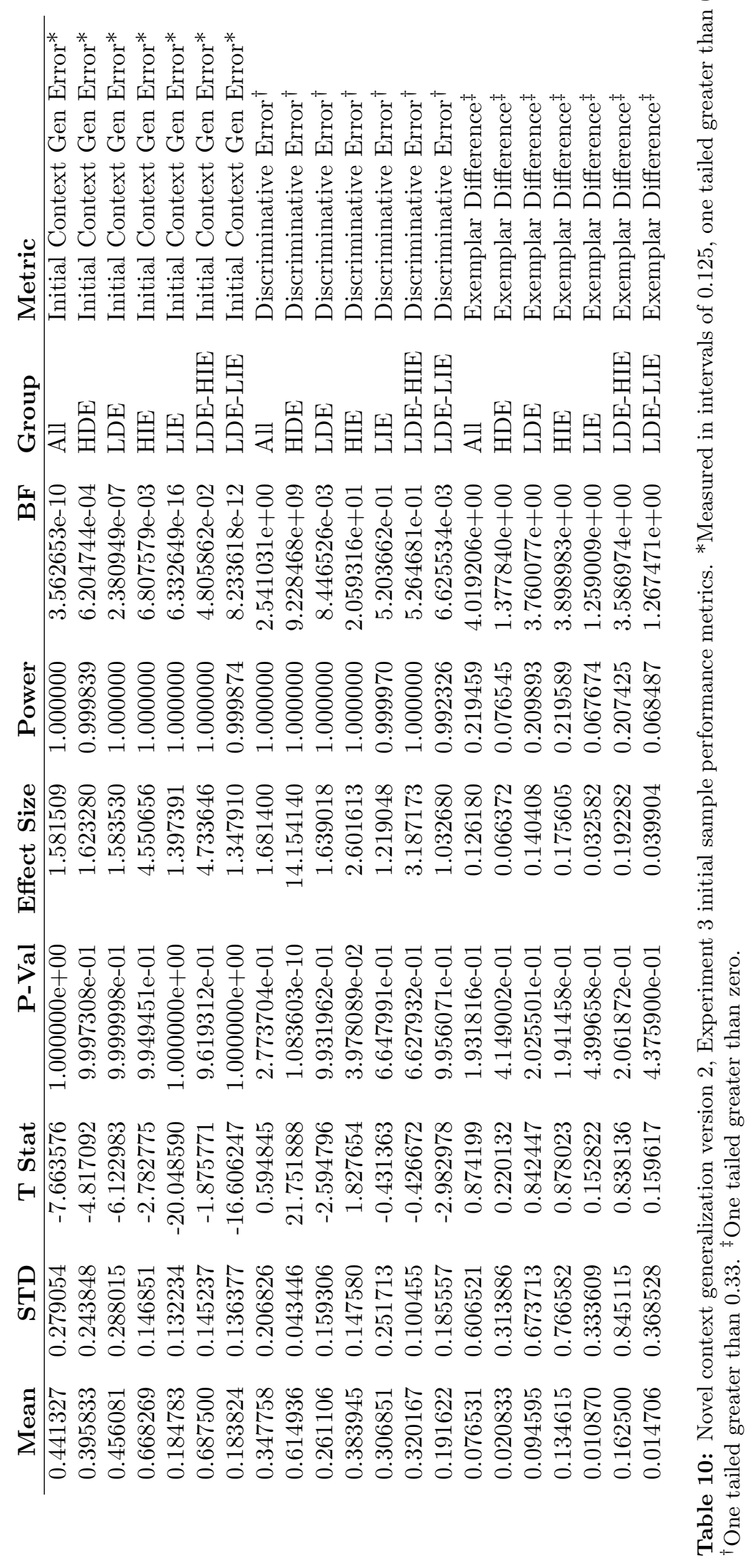




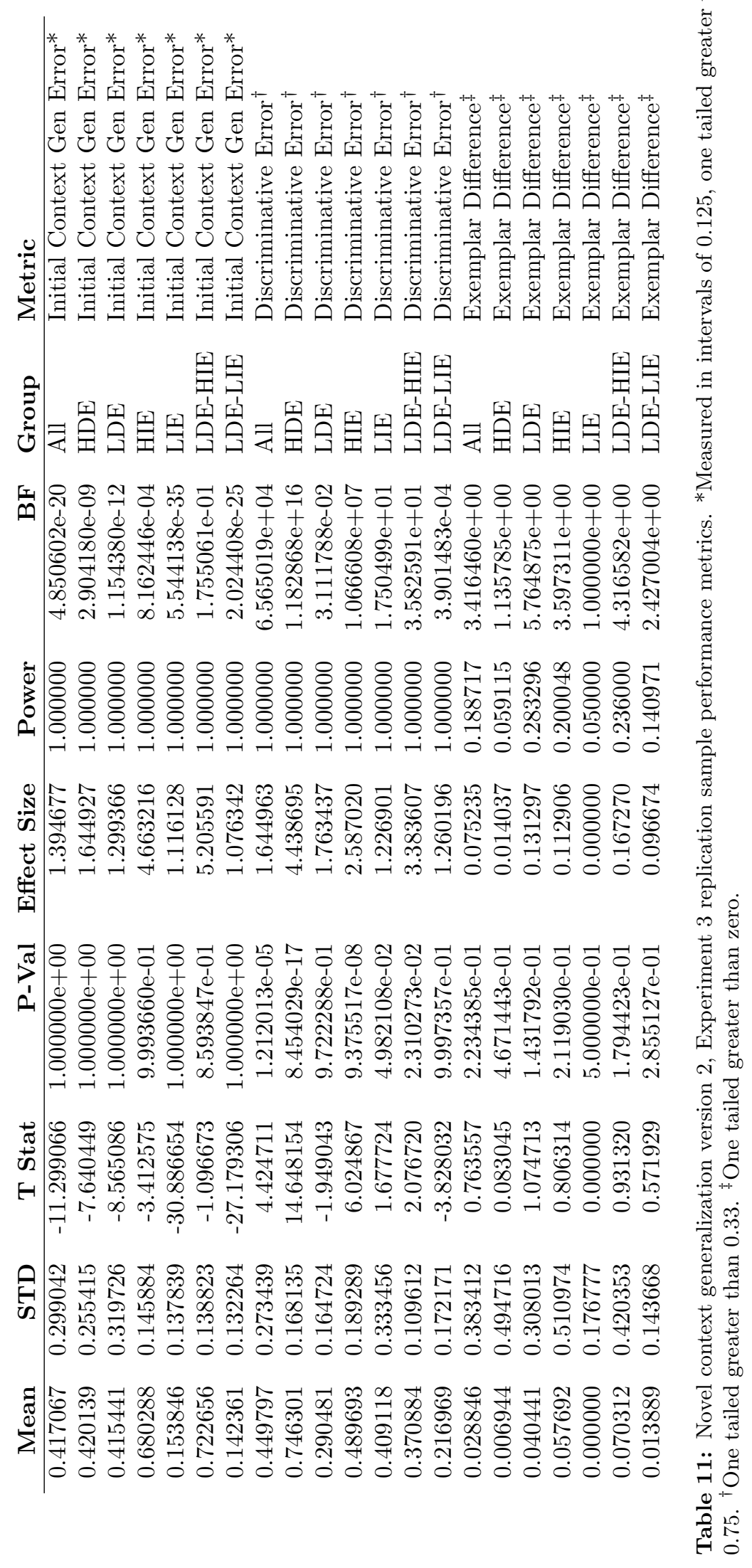




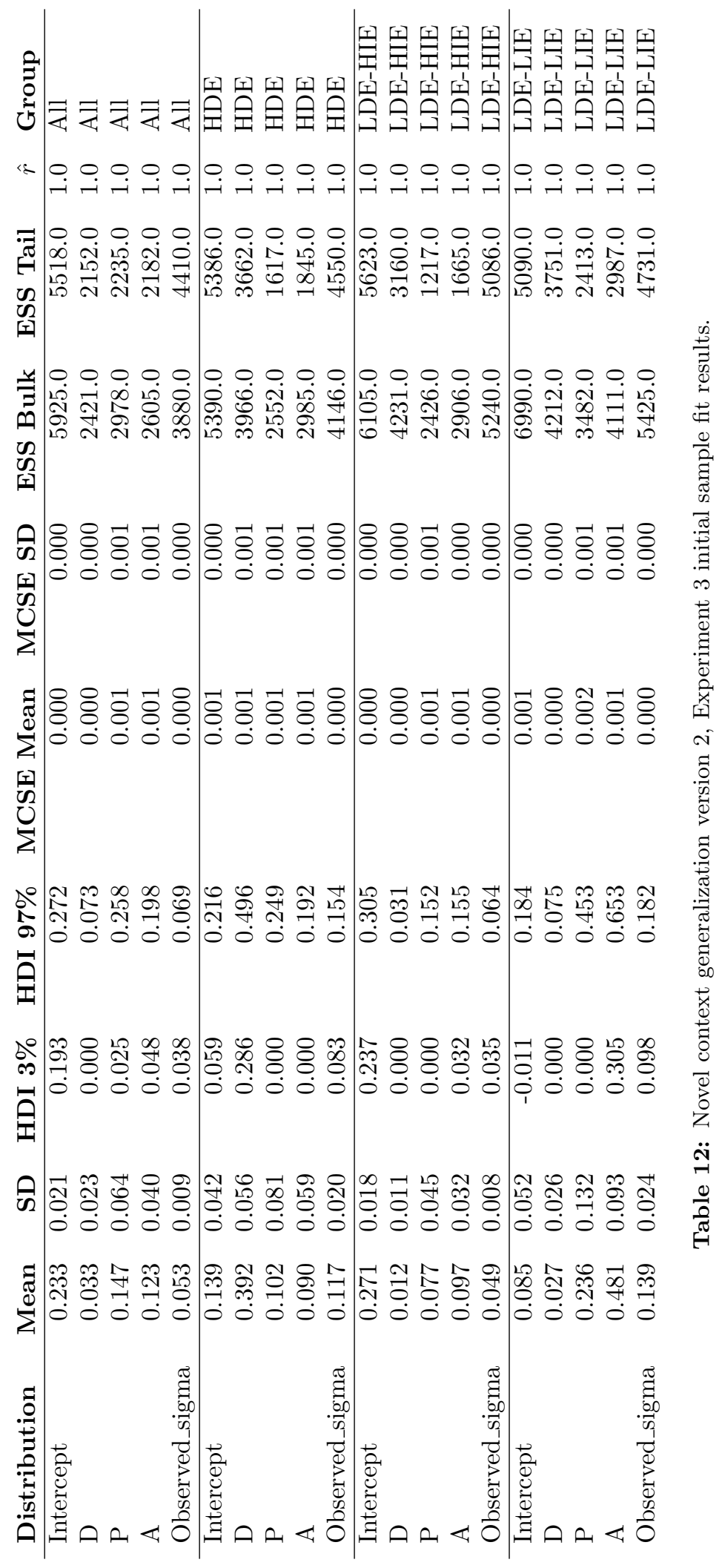




\begin{tabular}{lrrrl} 
Model & Rank & LOO & SE & Group \\
\hline No D & 0 & 35.245366 & 2.864782 & All \\
All & 1 & 34.713249 & 3.218341 & All \\
No P & 2 & 32.578324 & 3.389351 & All \\
No A & 3 & 31.576075 & 3.360691 & All \\
Null & 4 & 25.848300 & 2.844465 & All \\
\hline No P & 0 & 16.548009 & 3.312429 & HDE \\
No A & 1 & 16.089324 & 3.192851 & HDE \\
All & 2 & 16.058740 & 3.256250 & HDE \\
No D & 3 & 2.050339 & 2.922375 & HDE \\
Null & 4 & 1.157831 & 2.577550 & HDE \\
\hline No D & 0 & 38.194446 & 2.498972 & LDE-HIE \\
All & 1 & 37.329816 & 2.509104 & LDE-HIE \\
No P & 2 & 36.700421 & 2.536131 & LDE-HIE \\
No A & 3 & 33.758306 & 3.282707 & LDE-HIE \\
Null & 4 & 32.402980 & 2.875256 & LDE-HIE \\
\hline No D & 0 & 13.269654 & 3.284988 & LDE-LIE \\
All & 1 & 12.231403 & 3.194458 & LDE-LIE \\
No P & 2 & 11.378769 & 3.813237 & LDE-LIE \\
No A & 3 & 2.566141 & 2.586223 & LDE-LIE \\
Null & 4 & 0.580965 & 2.804869 & LDE-LIE
\end{tabular}

Table 13: Novel context generalization task version 2, initial sample model comparison. 


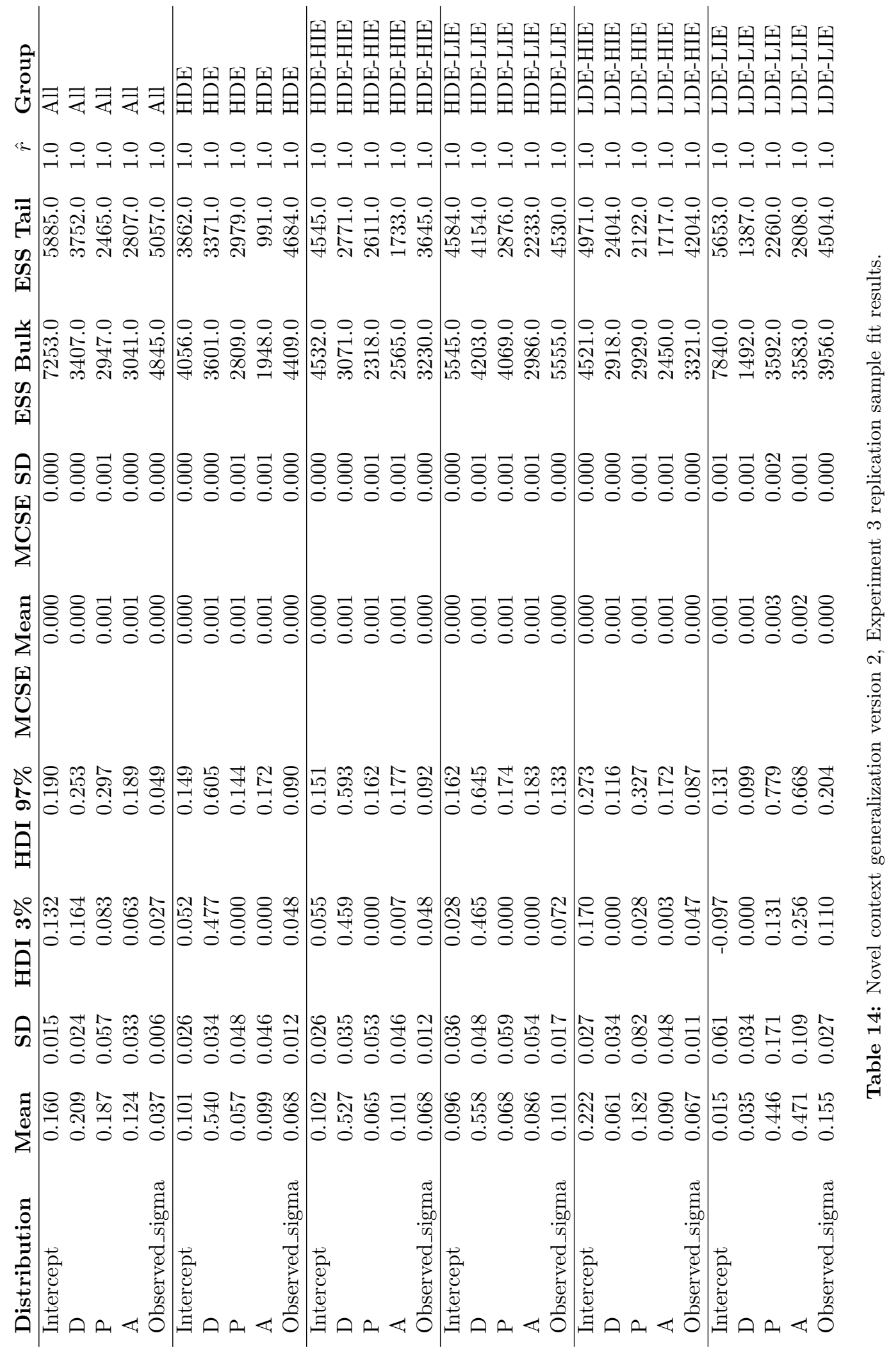




\begin{tabular}{lrrrl} 
Model & Rank & LOO & SE & Group \\
\hline All & 0 & 43.265087 & 2.613101 & All \\
No P & 1 & 38.577843 & 3.056587 & All \\
No A & 2 & 37.211161 & 2.867093 & All \\
No D & 3 & 23.387014 & 3.060166 & All \\
Null & 4 & 14.056161 & 1.939113 & All \\
\hline No P & 0 & 29.807506 & 3.603868 & HDE \\
All & 1 & 28.869283 & 3.395789 & HDE \\
No A & 2 & 26.618463 & 3.370051 & HDE \\
No D & 3 & -2.089074 & 2.094513 & HDE \\
Null & 4 & -3.323655 & 2.274451 & HDE \\
\hline No P & 0 & 29.925306 & 3.142845 & HDE-HIE \\
All & 1 & 29.117975 & 2.919238 & HDE-HIE \\
No A & 2 & 27.120274 & 2.633852 & HDE-HIE \\
No D & 3 & -1.330503 & 1.999877 & HDE-HIE \\
Null & 4 & -2.784847 & 2.136092 & HDE-HIE \\
\hline No P & 0 & 20.127741 & 4.535053 & HDE-LIE \\
All & 1 & 19.294702 & 4.331989 & HDE-LIE \\
No A & 2 & 18.675355 & 4.835028 & HDE-LIE \\
No D & 3 & -4.393554 & 2.505611 & HDE-LIE \\
Null & 4 & -4.974411 & 2.780662 & HDE-LIE \\
\hline No A & 0 & 29.274045 & 2.520767 & LDE-HIE \\
No D & 1 & 29.261423 & 3.469870 & LDE-HIE \\
All & 2 & 29.233640 & 2.868132 & LDE-HIE \\
No P & 3 & 27.569533 & 3.088763 & LDE-HIE \\
Null & 4 & 22.746446 & 2.290522 & LDE-HIE \\
\hline No D & 0 & 10.741747 & 3.080906 & LDE-LIE \\
All & 1 & 9.682107 & 2.951324 & LDE-LIE \\
No P & 2 & 6.325068 & 3.013107 & LDE-LIE \\
No A & 3 & 2.163405 & 2.248248 & LDE-LIE \\
Null & 4 & -2.618608 & 2.066741 & LDE-LIE
\end{tabular}

Table 15: Novel context generalization task version 2, Experiment 3 replication sample model comparison. 


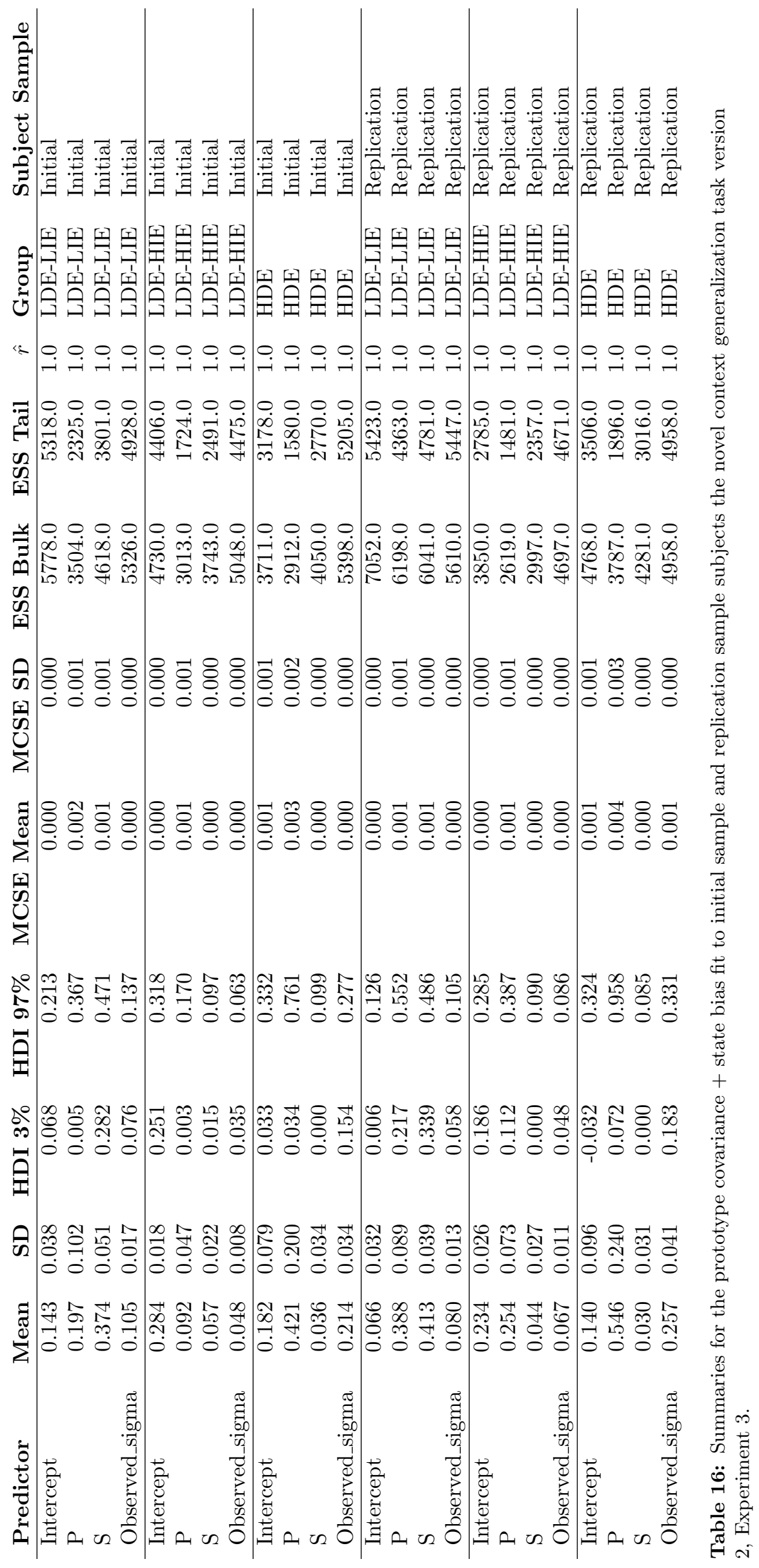




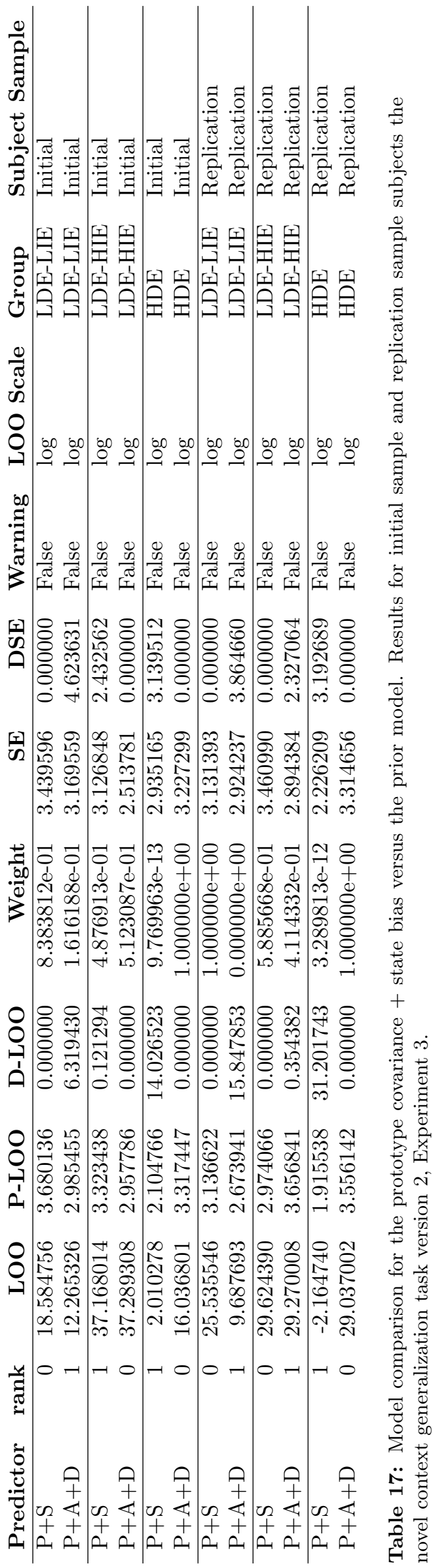




\begin{tabular}{lrrrl} 
Model & Rank & LOO & SE & Group \\
\hline No A & 0 & 26.360176 & 2.656569 & LDE-HIE Early \\
No D & 1 & 26.267128 & 2.952924 & LDE-HIE Early \\
All & 2 & 25.926387 & 2.667842 & LDE-HIE Early \\
No P & 3 & 23.900115 & 2.767347 & LDE-HIE Early \\
Null & 4 & 20.692428 & 2.143590 & LDE-HIE Early \\
\hline All & 0 & 26.985751 & 3.152952 & LDE-HIE Late \\
No D & 1 & 26.595123 & 3.270441 & LDE-HIE Late \\
No P & 2 & 26.557444 & 3.591234 & LDE-HIE Late \\
No A & 3 & 25.737913 & 2.468874 & LDE-HIE Late \\
Null & 4 & 22.055599 & 2.394200 & LDE-HIE Late \\
\hline No D & 0 & 5.750167 & 3.638409 & LDE-LIE Early \\
All & 1 & 4.855073 & 3.526805 & LDE-LIE Early \\
No P & 2 & 1.926536 & 3.216431 & LDE-LIE Early \\
No A & 3 & 1.185278 & 2.447104 & LDE-LIE Early \\
Null & 4 & -3.107404 & 1.970213 & LDE-LIE Early \\
\hline No D & 0 & 10.818845 & 2.801645 & LDE-LIE Late \\
All & 1 & 9.884811 & 3.055273 & LDE-LIE Late \\
No P & 2 & 8.202409 & 4.026801 & LDE-LIE Late \\
No A & 3 & -3.068803 & 3.567218 & LDE-LIE Late \\
Null & 4 & -5.861241 & 3.182447 & LDE-LIE Late
\end{tabular}

Table 18: Novel context generalization task version 2, Experiment 3 replication sample model comparison, early and late in the block. 


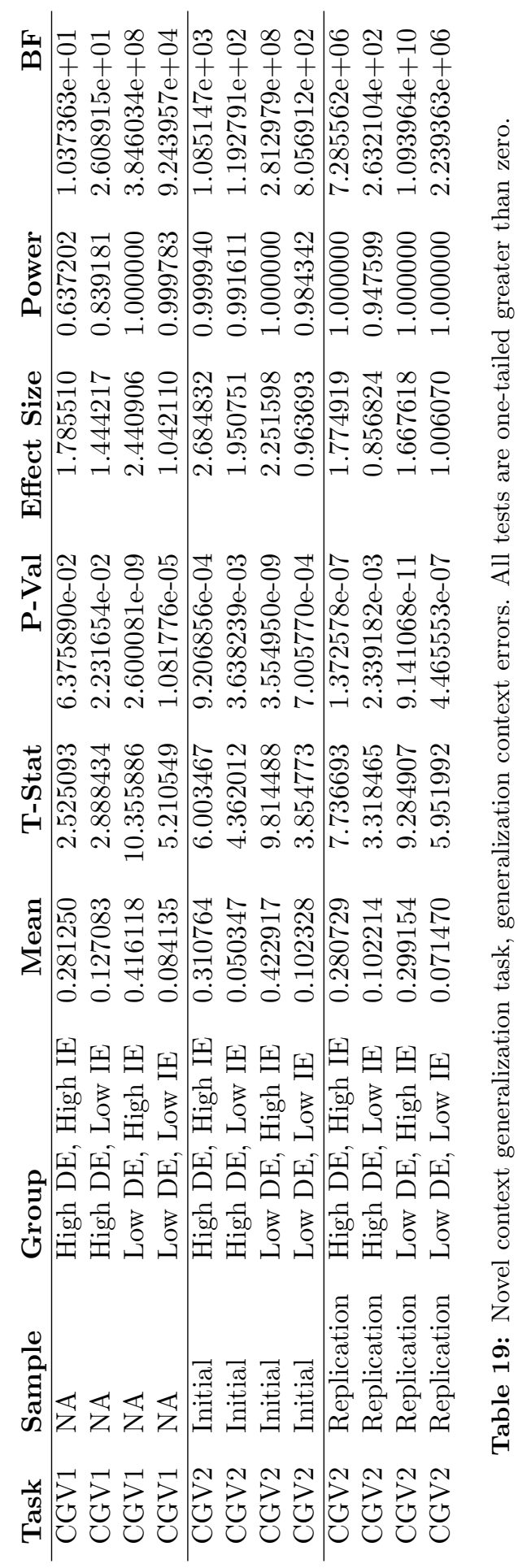




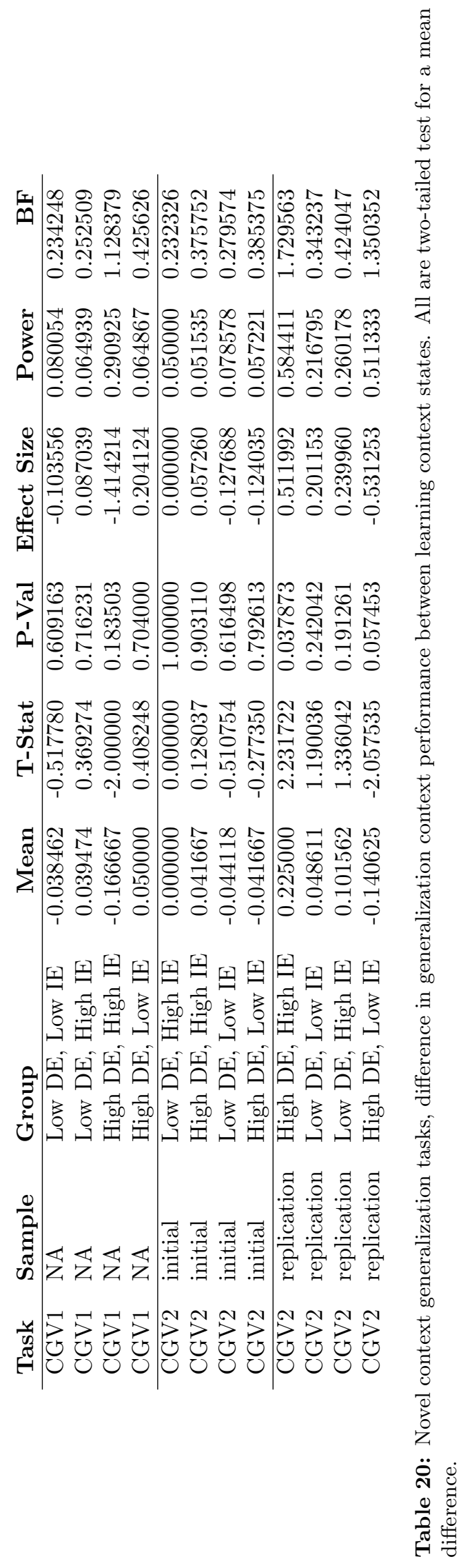

